\title{
Positive solutions for Schrödinger-Poisson type systems
}

\author{
Edwin Gonzalo Murcia Rodríguez
}

\author{
TESE APRESENTADA \\ $\mathrm{AO}$ \\ Instituto de Matemática e Estatística \\ $\mathrm{DA}$ \\ Universidade de SÃo Paulo \\ PARA \\ OBTENÇÃO DO TÍTULO \\ $\mathrm{DE}$ \\ Doutor EM CIÉnCIAS \\ Programa: Matemática \\ Orientador: Prof. Dr. Gaetano Siciliano
}

Durante o desenvolvimento deste trabalho o autor recebeu auxílio financeiro da CAPES

São Paulo, Junho de 2017 


\section{Positive solutions for Schrödinger-Poisson type systems}

Esta versão da tese contém as correções e alterações sugeridas pela Comissão Julgadora durante a defesa da versão original do trabalho, realizada em 09/06/2017. Uma cópia da versão original está disponível no Instituto de Matemática e Estatística da Universidade de São Paulo.

Comissão Julgadora:

- Prof. Dr. Gaetano Siciliano (orientador) - IME-USP

- Prof. Dr. Jaime Angulo Pava - IME-USP

- Prof. Dr. Ederson Moreira dos Santos - ICMC-USP

- Prof. Dr. Giovany de Jesus Malcher Figueiredo - UnB

- Prof. Dr. Stefano Nardulli - UFRJ 


\section{Resumo}

MURCIA, E. Soluções positivas para sistemas do tipo Schrödinger-Poisson. 2017. 68 f. Tese (Doutorado) - Instituto de Matemática e Estatística, Universidade de São Paulo, São Paulo, 2017.

Nesta tese nós estudamos sistemas de Schrödinger-Poisson e procuramos soluções positivas. Nosso trabalho consiste em três capítulos. O Capítulo 1 contém alguns fatos básicos sobre a teoria de pontos críticos. No Capítulo 2 nós consideramos um sistema fracionário de Schrödinger-Poisson em todo o espaço $\mathbb{R}^{N}$ em presença de um potencial positivo e que depende de um pequeno parâmetro positivo $\varepsilon$. Nós mostramos que, para $\varepsilon$ suficentemente pequeno (i.e. no limite semiclássico) o número de soluções positivas é estimado por abaixo pela categoria de Ljusternick-Schnirelmann dos conjuntos onde o potencial é mínimo. Finalmente, no Capítulo 3 nós analisamos um sistema Schrödinger-Poisson em $\mathbb{R}^{3}$ sob a não linearidade assintoticamente cúbica. Mostramos a existência de soluções radiais positivas dentro de uma bola e em um domínio exterior.

Palavras-chave: Métodos variacionais, sistema Schrödinger-Poisson, categoria de LjusternickSchnirelmann, no linearidade assintoticamente cúbica. 


\section{Abstract}

MURCIA, E. Positive solutions for Schrödinger-Poisson type systems. 2017. 68 p. Thesis (PhD) - Instituto de Matemática e Estatística, Universidade de São Paulo, São Paulo, 2017.

In this thesis we study Schrödinger-Poisson systems and we look for positive solutions. Our work consists in three chapters. Chapter 1 includes some basic facts on critical point theory. In Chapter 2 we consider a fractional Schrödinger-Poisson system in the whole space $\mathbb{R}^{N}$ in presence of a positive potential and depending on a small positive parameter $\varepsilon$. We show that, for suitably small $\varepsilon$ (i.e. in the "semiclassical limit") the number of positive solutions is estimated below by the Ljusternick-Schnirelmann category of the set of minima of the potential. Finally, in Chapter 3, we analyze a Schrödinger-Poisson system in $\mathbb{R}^{3}$ under an asymptotically cubic nonlinearity. We prove the existence of positive, radial solutions inside a ball and in an exterior domain.

Keywords: Variational methods, Schrödinger-Poisson system, Ljusternick-Schnirelmann category, Asymptotically cubic nonlinearity. 


\section{Contents}

Introduction $\quad$ vii

1 Preliminaries $\quad 1$

1.1 Functional spaces . . . . . . . . . . . . . . . . . . . . 1

1.2 Frequently used results . . . . . . . . . . . . . . . . . . . 2

1.3 Some facts about calculus in Banach spaces . . . . . . . . . . . . . . . 5

1.4 The reduction argument for the Schrödinger-Poisson system . . . . . . . . . . . . . . . . .

1.5 A brief summary of the theory of Hilbert manifolds . . . . . . . . . . . . . . 13

1.6 The Ljusternick-Schnirelmann category . . . . . . . . . . . . . . . . . . . . . . 19

2 Positive semiclassical states for a fractional Schrödinger-Poisson system $\quad 27$

2.1 Introduction . . . . . . . . . . . . . . . . . . . . 27

2.2 Preliminaries . . . . . . . . . . . . . . . . . . . . . . . . . . . 29

2.2 .1 Some well known facts . . . . . . . . . . . . . . . . . . . . . . . 29

2.2 .2 The variational setting . . . . . . . . . . . . . . . . 30

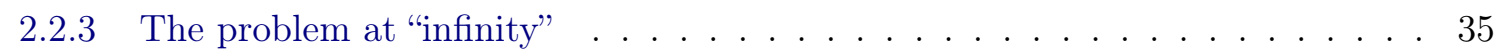

2.3 Compactness properties for the functionals $I_{\varepsilon}, E_{\mu} \ldots \ldots \ldots \ldots$

2.4 Proof of the main theorem . . . . . . . . . . . . . . . . . . . . . 41

3 Positive solutions to the Schrödinger-Poisson system under asymptotically cubic $\begin{array}{ll}\text { nonlinearity } & 49\end{array}$

3.1 Introduction . . . . . . . . . . . . . . . . . . . . . . . . . . 49

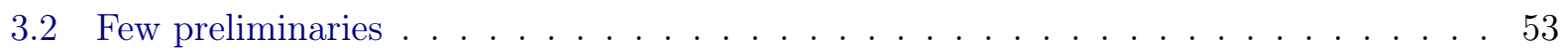

3.3 A positive radial solution in the complementary of a ball . . . . . . . . . . . 55

3.3.1 Mountain Pass geometry of $I_{\mathbb{R}^{3} \backslash B_{R}} \ldots \ldots \ldots \ldots \ldots \ldots$

3.3.2 Compactness condition for $I_{\mathbb{R}^{3} \backslash B_{R}} \ldots \ldots \ldots \ldots \ldots \ldots$

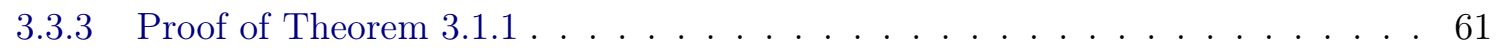

3.4 A positive radial solution inside the ball . . . . . . . . . . . . . . . . 62

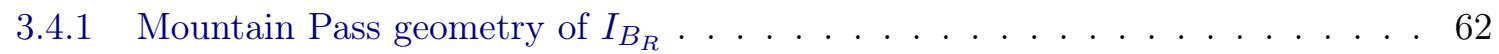

3.4 .2 Compactness condition for $I_{B_{R}} \ldots \ldots \ldots \ldots \ldots \ldots \ldots \ldots \ldots \ldots \ldots \ldots$

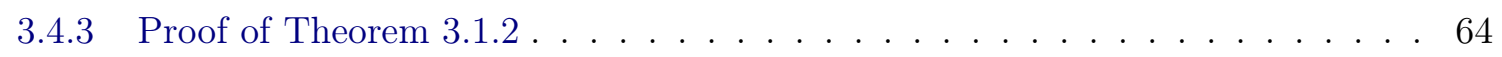

$\begin{array}{ll}\text { Bibliography } & 65\end{array}$ 
vi CONTENTS 


\section{Introduction}

In this thesis we study some Schrödinger-Poisson equations in different contexts. Chapter 1 includes some frequently used results in the whole research. Chapter 2 contains a study about a fractional Schrödinger-Poisson system, and the main result is about the existence of multiple positive solutions. Finally, in Chapter 3, we analyze the behavior of a Schrödinger-Poisson system in $\mathbb{R}^{3}$ under an asymptotically cubic nonlinearity. We prove the existence of positive, radial solutions inside a ball and in an exterior domain. Now we give a more detailed description of the contents of the chapters of this thesis.

In Chapter 1 we collect some of the elementary ingredients used in the whole research. We open the chapter with the Sobolev spaces, giving a brief description of their definitions, norms and the important results about continuous and compact embeddings in $L^{p}$ spaces. Naturally we include the notation that will be used through the thesis. Some concrete notations will be postponed to the appropriate place within the exposition of the topics. After the statement of the main theorems from the Measure Theory used in our work, we continue with a rudimentary explanation of the elements of the Calculus in Banach spaces. It is worthwhile to mention that the concept of Fréchét derivative plays a crucial role in our approach to the Schrödinger-Poisson systems. Indeed it is a prerequisite for the notion of critical point. By the way, usually it is more easy to start with the Gâteaux derivative of a functional, and then proving that it coincides with the Fréchét derivative of the functional; the conditions for this equality are given in this part of the chapter. The next section deals with a reduction argument for Schrödinger-Poisson systems via the Riesz representation theorem. Roughly speaking we can solve a Schrödinger-Poisson system by studying a functional that depends on a single variable whereas, in the first instance, the natural way to solve the system relies on the study of a functional that depends on two independent variables. The Riesz theorem is the key tool that simplifies the situation. The following topic in the chapter is the theory of Hilbert manifolds; for us, manifolds of codimension one and, more concretely, manifolds that are natural constraints for a functional will be fundamental. As an example, the Nehari manifold has a remarkable role in Chapter 2. The last section of Chapter 1 considers the category of LjusternickSchnirelmann. After some definitions and properties, the main result of the category guarantees multiplicity of critical points for a functional on a suitable Hilbert manifold, when the functional has two important properties: compactness (i.e. it satisfies the $(P S)$ condition) and boundedness from below.

In Chapter 2 we study a fractional Schrödinger-Poisson system, and we obtain a result of multiplicity of positive solutions via Ljusternick-Schnirelmann theory, when a positive parameter of the system approaches to zero (this feature gives the adjective of "semiclassical" to the solutions). The system is fractional because the laplacian operator involved in the two equations is also fractional: if it acts on a function $u$, the more general definition of the operator is given in terms of the Fourier 
transform of $u$. When $u$ is smooth enough, the action can be expressed as an integral. The system also contains a term with a continuous scalar field $V$, which has the physical meaning of an external potential, and a general continuous nonlinearity $f$. The scalar field $V$ is relevant because the whole work is based on the topological properties of the set where this potential has a minimum. Among the assumptions on $f$, we remark the so called Ambrosetti-Rabinowitz condition, of ample use in similar works. This condition is important, for example, to have a desirable geometry for the functionals. The power types of nonlinearity $|u|^{p-2} u$ are included in this kind of nonlinearity $f$. After the definition of the fractional and homogeneous Sobolev spaces, that are the natural functional spaces to consider, we state the variational setting of the problem: first we apply the reduction argument of the system using the Riesz theorem, and then we conclude that the fractional system can be solved employing Critical Point theory. In the process an "auxiliary" problem will be relevant, and the functionals involved will have a suitable geometry and we will be able to define the notion of Nehari manifold for these functionals.

In the next step we pursue compactness. In our context, this means that the Palais-Smale sequences for the functional have good properties. To start with, we show that they are bounded. Nevertheless, the eventual behavior of the external potential $V$ will have a prominent role: this behavior will determine when the weak convergence fails and, as a consequence, it will determine under which conditions the Palais-Smale sequences for the free and the constrained functionals (i.e., the functional restricted to the Nehari manifold) will have a convergent subsequence. This part of the chapter ends with the existence of a least energy solution for the problem. Although it is a byproduct, it is the most difficult result to prove in this chapter.

Finally we prove the result of multiplicity of solutions. For this, we relate the topological complexity of certain sublevels of the functional restricted to the Nehari manifold (i.e., the set of values of the functional in functions on the manifold that are below a given real level) with the topological properties of the set where the external potential $V$ achieves the minimum. The relationship is given by the Ljusternick-Schnirelmann category. Furthermore, when this set associated to $V$ is not trivial (in a topological sense), we have one more solution to the system.

Finally, in Chapter 3 we study a Schrödinger-Poisson system of type

$$
\begin{cases}-\Delta u+u+\lambda \phi u=f(u) & \text { in } \Omega \\ -\Delta \phi=\widetilde{u}^{2} & \text { in } \mathbb{R}^{3} \\ u=0 & \text { on } \partial \Omega\end{cases}
$$

where $\Omega$ is a domain (for us the interesting cases are a ball centered at the origin with radius $R$, $B_{R}$, and its complementary), $\lambda>0$ is a parameter, $f$ is a special nonlinearity and a solution is a pair of functions $(u, \phi)$. The function $u$ is defined in $\Omega$ (actually, it is a member of a Sobolev space on $\Omega$, as it will be seen) and $\widetilde{u}$ is its trivial extension to the whole $\mathbb{R}^{3}$, i.e., $\widetilde{u}=0$ outside $\Omega$. The most relevant feature about $f$ is that it has an eventually "subcubic" behaviour. This asymptotic behaviour represents difficulties because, in particular, it implies that $f$ does not satisfy the Ambrosetti-Rabinowitz condition. Furthermore, it induces a "competition" between the terms $\lambda \phi$ and $f$ in the context that it is explained in Chapter 3. Again, the reduction argument can be used here to analyze the system. The main results of this chapter are the existence of positive, radial solutions to the problems with $\Omega=B_{R}$ and $\Omega=\mathbb{R}^{3} \backslash B_{R}$, under suitable conditions on $\lambda$ and $R$. 
For the system with $\Omega=\mathbb{R}^{3} \backslash B_{R}$, the associated functional has the mountain pass geometry, and the difficult part there consists of finding a function with large norm for which the functional has a negative value. An existence result about a positive, radial solution for a Berestycki-Lions-type problem in the whole $\mathbb{R}^{3}$ is used to this purpose. The required compactness is guaranteed in terms of a special type of sequences, the Cerami sequences.

For the system with $\Omega=B_{R}$, the associated functional also satisfies the mountain pass geometry, and the compactness condition holds for the Palais-Smale sequences. 


\section{Chapter 1}

\section{Preliminaries}

In this chapter, we introduce some tools needed to understand the results obtained in our research. First, we mention explicitly the facts that occurs more frequently in our work. Then we give a brief introduction of the calculus in Banach spaces, and its application to a variational problem. After that, we focus on a "reduction argument" that will be used in the Chapters 2 and 3. The byproduct of this treatment is that we can illustrate some of the standard techniques, which are frequently used to study these type of systems. Then we introduce some elements of the theory of Hilbert manifolds. Finally, we briefly expose the Ljusternick-Schnirelmann theory, that will be fundamental in order to obtain our result about multiplicity of solutions in the Chapter 2.

\section{$1.1 \quad$ Functional spaces}

If $\Omega \subset \mathbb{R}^{3}$ is a domain, $L^{p}(\Omega)$ is the usual Lebesgue space endowed with norm $|\cdot|_{p, \Omega} . H^{1}(\Omega)$ is the Hilbert space endowed with the inner product and norm

$$
\langle u, v\rangle_{\Omega}:=\int_{\Omega} \nabla u \cdot \nabla v d x+\int_{\Omega} u v d x, \quad\|u\|_{\Omega}^{2}:=\langle u, u\rangle_{\Omega}
$$

The space $H_{0}^{1}(\Omega)$ is the subspace of $H^{1}(\Omega)$ defined as the completion of the test functions with respect to $\|\cdot\|_{\Omega}$. When $\Omega \subset \mathbb{R}^{3}$ is a bounded domain, $H_{0}^{1}(\Omega)$ is a Hilbert space endowed with the equivalent inner product and norm given by

$$
\langle u, v\rangle_{H_{0}^{1}(\Omega)}:=\int_{\Omega} \nabla u \cdot \nabla v d x, \quad\|u\|_{H_{0}^{1}(\Omega)}^{2}:=\langle u, u\rangle_{H_{0}^{1}(\Omega)}
$$

If $\Omega=\mathbb{R}^{3}$ we will suppress the symbol $\Omega$ in scalar products and Sobolev norms, as well as in the $L^{p}$-norm. We will make use also of the Hilbert space

$$
D^{1,2}\left(\mathbb{R}^{3}\right)=\left\{u \in L^{6}\left(\mathbb{R}^{3}\right):|\nabla u| \in L^{2}\left(\mathbb{R}^{3}\right)\right\},
$$

which coincides with the completion of $C_{c}^{\infty}\left(\mathbb{R}^{3}\right)$, the space of infinitely differentiable functions with compact support, with respect to the (square) norm

$$
\|u\|_{D}^{2}:=\int_{\mathbb{R}^{3}}|\nabla u|^{2} d x
$$

The dual spaces of $H_{0}^{1}(\Omega)$ and $D^{1,2}\left(\mathbb{R}^{3}\right)$ are denoted by $H^{-1}$ and $D^{\prime}$, respectively. For these spaces, we have the following continuous and compact embedding results. Recall that given $X, Y$ Banach spaces, the notation $X \hookrightarrow Y$ means that $X$ is continuously embedded into $Y$, i.e. $X \subset Y$ and the inclusion map $\iota: X \rightarrow Y$ is continuous. In this case, there exists a constant $C>0$ such that

$$
\|u\|_{Y} \leq C\|u\|_{X}, \quad \forall u \in X
$$


When $X \hookrightarrow Y$ and the inclusion map $\iota$ is also a compact map we say that $X$ is compactly embedded into $Y$ and we use the notation $X \hookrightarrow \hookrightarrow Y$. See Ambrosetti and Malchiodi (2007, pg. 4) and Badiale and Serra (2011, pg. 6-7) for more details.

Theorem 1.1.1. If $\Omega \subset \mathbb{R}^{N}$ is a bounded domain, for $N \geq 3$ we have

$$
H^{1}(\Omega) \hookrightarrow L^{p}(\Omega), \quad \text { for } 1 \leq p \leq 2^{*}:=\frac{2 N}{N-2}
$$

and

$$
H^{1}(\Omega) \hookrightarrow \hookrightarrow L^{p}(\Omega), \quad \text { for } 1 \leq p<2^{*}
$$

In the case $\Omega=\mathbb{R}^{N}$,

$$
H^{1}\left(\mathbb{R}^{N}\right) \hookrightarrow L^{p}\left(\mathbb{R}^{N}\right), \quad \text { for } 2 \leq p \leq 2^{*}
$$

and

$$
D^{1,2}\left(\mathbb{R}^{N}\right) \hookrightarrow L^{2^{*}}\left(\mathbb{R}^{N}\right)
$$

The embeddings in the whole space are not compact.

We use the symbols $C, C_{i}$, for $i=1,2, \ldots$ for positive constants which may also change from line to line.

\subsection{Frequently used results}

We state two results of measure theory often used in this thesis. See Royden (1988, pg. 86 and pg. 91) for more details.

Theorem 1.2.1 (Fatou's lemma). Let $\left\{f_{n}\right\}$ be a sequence of nonnegative, measurable functions on $\Omega$, such that $f_{n}(x) \rightarrow f(x)$ a.e. in $\Omega$. Then

$$
\int_{\Omega} f d x \leq \liminf _{n \rightarrow \infty} \int_{\Omega} f_{n} d x
$$

Theorem 1.2.2 (Lebesgue Convergence Theorem). Let $g$ be integrable over $\Omega$ and let $\left\{f_{n}\right\}$ be a sequence of measurable functions such that $\left|f_{n}\right| \leq g$ on $\Omega$ and for almost all $x$ in $\Omega$ we have $f_{n}(x) \rightarrow f(x)$ when $n \rightarrow \infty$. Then

$$
\int_{\Omega} f_{n} d x \rightarrow \int_{\Omega} f d x
$$

Now we give a pertinent example where we apply the previous theorem. For this example we are going to use Young's inequality, i.e.,

$$
\text { if } a, b>0 \text { and } p, q>1 \text { are numbers such that } \frac{1}{p}+\frac{1}{q}=1 \text {, then } a b \leq \frac{a^{p}}{p}+\frac{b^{q}}{q} \text {. }
$$

Example 1.2.3. Let $\Omega \subset \mathbb{R}^{3}$ be a radial domain with $\partial \Omega$ smooth, and let $f \in C(\mathbb{R}, \mathbb{R})$ be a function such that

$$
\lim _{t \rightarrow 0} \frac{f(t)}{t}=0 \quad \text { and } \quad \exists q \in(4,6) \text { s.t. } \lim _{|t| \rightarrow \infty} \frac{f(t)}{t^{q-1}}=0 .
$$

We emphasize that the following particular case satisfies the previous conditions; it will be used in Chapter 3 ( $B_{R}$ denotes the ball in $\mathbb{R}^{3}$ with center at the origin and radius $R>0$ ):

$$
\Omega=\mathbb{R}^{3} \backslash B_{R}, \quad f \in C(\mathbb{R}, \mathbb{R}), \quad f(t)=0 \text { for } t \leq 0, \quad \lim _{t \rightarrow 0} \frac{f(t)}{t}=0 \quad \text { and } \quad \lim _{t \rightarrow \infty} \frac{f(t)}{t^{3}}=1 .
$$

Denoting $F(t):=\int_{0}^{t} f(\tau) d \tau$, it can be shown that given $\varepsilon>0$, there is a constant $M_{\varepsilon}>0$ such that

$$
f(t) \leq \varepsilon|t|+M_{\varepsilon}|t|^{q-1} \quad \text { and } \quad F(t) \leq \frac{\varepsilon}{2} t^{2}+\frac{M_{\varepsilon}}{q}|t|^{q}, \quad \forall t \in \mathbb{R} .
$$


Our purpose in this example is to show that, if $\left\{u_{n}\right\} \subset H_{0, \text { rad }}^{1}(\Omega)$ satisfies $u_{n} \rightarrow u$ in $H_{0, \text { rad }}^{1}(\Omega)$, when $n \rightarrow \infty$ we have

$$
\int_{\Omega} f\left(u_{n}\right) w d x \rightarrow \int_{\Omega} f(u) w d x, \int_{\Omega} F\left(u_{n}\right) d x \rightarrow \int_{\Omega} F(u) d x \text { and } \int_{\Omega} f\left(u_{n}\right) u_{n} d x \rightarrow \int_{\Omega} f(u) u d x,
$$

for every $w \in H_{0, \text { rad }}^{1}(\Omega)$. In fact, since $H_{0, \text { rad }}^{1}(\Omega) \hookrightarrow \hookrightarrow L^{p}(\Omega)$ with $2<p<6$, up to a subsequence

$$
\begin{aligned}
& u_{n} \rightarrow u \text { in } L^{p}(\Omega), 2<p<6 \\
& u_{n} \rightarrow u \text { a.e. } \Omega
\end{aligned}
$$

We follow some of the ideas given in Alves et al. (2004, Lemma 3.1). Using (1.2.1) for $\varepsilon>0$ fixed but arbitrary,

$$
\begin{aligned}
\left|\left[f\left(u_{n}\right)-f(u)\right] w\right| & \leq \varepsilon\left|u_{n} w\right|+M_{\varepsilon}\left|u_{n}\right|^{q-1} w+\varepsilon|u w|+M_{\varepsilon}|u|^{q-1}|w| \\
& \leq C\left[\varepsilon\left(u_{n}^{2}+w^{2}\right)+M_{\varepsilon}\left(\left|u_{n}\right|^{q}+|w|^{q}\right)+\varepsilon\left(u^{2}+w^{2}\right)+M_{\varepsilon}\left(|u|^{q}+|w|^{q}\right)\right] \\
& =C\left[\left(\varepsilon u_{n}^{2}+M_{\varepsilon}\left|u_{n}\right|^{q}\right)+\varepsilon\left(u^{2}+2 w^{2}\right)+M_{\varepsilon}\left(|u|^{q}+2|w|^{q}\right)\right],
\end{aligned}
$$

where $C>0$ is a constant. Denoting $\varepsilon:=C \varepsilon, M_{\varepsilon}:=C M_{\varepsilon}$, we define the auxiliary function

$$
g_{\varepsilon}\left(u_{n}\right):=\max \left\{0,\left|\left[f\left(u_{n}\right)-f(u)\right] w\right|+\varepsilon\left(u^{2}-u_{n}^{2}\right)+M_{\varepsilon}\left(|u|^{q}-\left|u_{n}\right|^{q}\right)\right\} .
$$

It satisfies, when $n \rightarrow \infty$

$$
g_{\varepsilon}\left(u_{n}\right) \rightarrow 0 \text { a.e. } \Omega, \text { because } u_{n} \rightarrow u \text { a.e. } \Omega
$$

and

$$
0 \leq g_{\varepsilon}\left(u_{n}\right) \leq 2 \varepsilon\left(u^{2}+w^{2}\right)+2 M_{\varepsilon}\left(|u|^{q}+|w|^{q}\right) \in L^{1}(\Omega),
$$

so that, by Lebesgue theorem

$$
\int_{\Omega} g_{\varepsilon}\left(u_{n}\right) d x \rightarrow 0 \quad \text { when } \quad n \rightarrow \infty
$$

Since

$$
\left|\left[f\left(u_{n}\right)-f(u)\right] w\right| \leq \varepsilon\left(u_{n}^{2}+u^{2}\right)+M_{\varepsilon}\left(\left|u_{n}\right|^{q}-|u|^{q}\right)+g_{\varepsilon}\left(u_{n}\right),
$$

we have

$$
\begin{aligned}
\int_{\Omega}\left|\left[f\left(u_{n}\right)-f(u)\right] w\right| d x & \leq \varepsilon \int_{\Omega}\left(u_{n}^{2}+u^{2}\right) d x+M_{\varepsilon}\left(\int_{\Omega}\left|u_{n}\right|^{q} d x-\int_{\Omega}|u|^{q} d x\right)+\int_{\Omega} g_{\varepsilon}\left(u_{n}\right) d x \\
& \leq \varepsilon\left(C+\int_{\Omega} u^{2} d x\right)+o_{n}(1),
\end{aligned}
$$

from which

$$
\limsup _{n \rightarrow \infty} \int_{\Omega}\left|\left[f\left(u_{n}\right)-f(u)\right] w\right| d x \leq C \varepsilon, \text { for } \varepsilon>0 \text { fixed. }
$$

By the arbitrariness of $\varepsilon$

$$
\limsup _{n \rightarrow \infty} \int_{\Omega}\left|\left[f\left(u_{n}\right)-f(u)\right] w\right| d x=0
$$

Similarly, from

$$
\left|f\left(u_{n}\right) u_{n}-f(u) u\right| \leq \varepsilon u_{n}^{2}+M_{\varepsilon}\left|u_{n}\right|^{q}+\varepsilon u^{2}+M_{\varepsilon}|u|^{q}
$$

we define, for $\varepsilon>0$ fixed but arbitrary, the function

$$
h_{\varepsilon}\left(u_{n}\right):=\max \left\{0,\left|f\left(u_{n}\right) u_{n}-f(u) u\right|+\varepsilon\left(u^{2}-u_{n}^{2}\right)+M_{\varepsilon}\left(|u|^{q}-\left|u_{n}\right|^{q}\right)\right\}
$$


which satisfies

$$
h_{\varepsilon}\left(u_{n}\right) \rightarrow 0 \text { a.e. } \Omega \text { and } h_{\varepsilon}\left(u_{n}\right) \leq 2\left(\varepsilon u^{2}+M_{\varepsilon}|u|^{q}\right) \in L^{1}(\Omega) .
$$

Thus, by Lebesgue theorem

$$
\lim _{n \rightarrow \infty} \int_{\Omega} h\left(u_{n}\right) d x=0
$$

Since

$$
\left|f\left(u_{n}\right) u_{n}-f(u) u\right| \leq \varepsilon\left(u_{n}^{2}+u^{2}\right)+M_{\varepsilon}\left(\left|u_{n}\right|^{q}-|u|^{q}\right)+h_{\varepsilon}\left(u_{n}\right),
$$

after integrating on $\Omega$

$$
\int_{\Omega}\left|f\left(u_{n}\right) u_{n}-f(u) u\right| d x \leq \varepsilon\left(C+\int_{\Omega} u^{2} d x\right)+o_{n}(1) .
$$

Therefore, by the arbitrariness of $\varepsilon$

$$
\limsup _{n \rightarrow \infty} \int_{\Omega}\left|f\left(u_{n}\right) u_{n}-f(u) u\right| d x=0 .
$$

Using the function

$$
k_{\varepsilon}\left(u_{n}\right):=\max \left\{0,\left|F\left(u_{n}\right)-F(u)\right|+\varepsilon\left(u^{2}-u_{n}^{2}\right)+M_{\varepsilon}\left(|u|^{q}-\left|u_{n}\right|^{q}\right)\right\},
$$

a completely analogous argument give us

$$
\limsup _{n \rightarrow \infty} \int_{\Omega}\left|F\left(u_{n}\right)-F(u)\right| d x=0,
$$

and from (1.2.3), (1.2.4) and (1.2.5) we conclude (1.2.2).

Lemma 1.2.4 (Brezis-Lieb lemma). Given $1<p<\infty$, if $\left\{f_{n}\right\} \subset L^{p}(\Omega)$ is a bounded sequence of functions such that $f_{n}(x) \rightarrow f(x)$ a.e. $x \in \Omega$, then $f \in L^{p}(\Omega)$ and

$$
\int_{\Omega}\left(\left|f_{n}\right|^{p}-\left|f_{n}-f\right|^{p}\right) d x \rightarrow \int_{\Omega}|f|^{p} d x
$$

In virtue of the Brezis-Lieb lemma, if $\left\{f_{n}\right\} \subset L^{p}(\Omega)$ is a sequence of functions such that

$$
f_{n}(x) \rightarrow f(x) \text { a.e. } x \in \Omega \text { and }\left\|f_{n}\right\|_{L^{p}(\Omega)} \rightarrow\|f\|_{L^{p}(\Omega)},
$$

then

$$
f_{n} \rightarrow f \text { in } L^{p}(\Omega) .
$$

Now we state a theorem for Hilbert spaces, that will be used very often in this thesis. See Badiale and Serra (2011, Theorem 1.2.9).

Theorem 1.2.5 (Riesz representation theorem). Let $H$ be a real Hilbert space with an inner product $(\cdot, \cdot)$, that induces a norm $\|\cdot\|$ on $H$. Denote by $H^{\prime}$ the topological dual of $H$; it is endowed with a norm $\|\cdot\|_{\star}$. Then, for every $f \in H^{\prime}$, there is a unique element $u_{f} \in H$ that satisfies

$$
\left(u_{f}, v\right)=f(v), \quad \text { for all } v \in H
$$

and

$$
\|f\|_{\star}=\left\|u_{f}\right\| .
$$

Thus, there exists a bijective map $R: H^{\prime} \rightarrow H$ defined by $R(f):=u_{f}$. 


\subsection{Some facts about calculus in Banach spaces}

Let $\left(X,\|\cdot\|_{X}\right),\left(Y,\|\cdot\|_{Y}\right)$ be Banach spaces. We denote by $L(X, Y)$ the set of linear, continuous maps from $X$ to $Y . L(X, Y)$ has also a Banach space structure with the norm

$$
\|A\|_{\star}:=\sup _{x \in X,\|x\|_{X} \leq 1}\|A[x]\|_{Y}
$$

We can define the concept of differentiable map, in complete analogy with the case of calculus in $\mathbb{R}^{n}$.

Definition 1.3.1. Let $U \subset X$ be an open set, and $f: U \rightarrow Y$ a map. The function $f$ is (Fréchét) differentiable at $u \in U$ if there is $A \in L(X, Y)$ such that

for $\quad R(h):=f(x+h)-f(x)-A[h], \quad$ with $h \in X$ with $x+h \in U, \quad R(h)=o\left(\|h\|_{X}\right)$.

Recall that $R(h)=o\left(\|h\|_{X}\right)$ means

$$
\frac{\|R(h)\|_{Y}}{\|h\|_{X}} \rightarrow 0 \quad \text { when } \quad\|h\|_{X} \rightarrow 0 .
$$

Some remarks about this definition:

- $A$ in the previous definition is unique; we denote $f^{\prime}(u):=A$.

- If $f$ is differentiable at $u$, then $f$ is continuous at $u$.

- If $f \in L(X, Y)$, then $f$ is differentiable at every point $u$, and $f^{\prime}(u)=f$.

- If $f$ is differentiable at every point $u \in U$, we say that $f$ is differentiable in $U$.

- When $f$ is differentiable in $U$, if the map $f^{\prime}: U \rightarrow L(X, Y)$ is continuous, we say that $f$ is $C^{1}$, or more specifically, $f \in C^{1}(U, Y)$.

- When $f^{\prime}: U \subset X \rightarrow L(X, Y)$ is differentiable at $u$, we say that $f$ is twice differentiable at $u$, and we denote $f^{\prime \prime}(u):=\left(f^{\prime}\right)^{\prime}(u)$. Note that $f^{\prime \prime}(u) \in L(X, L(X, Y)) \simeq L(X \times X, Y)$, where $L(X \times X, Y)$ is the set of bilinear, continuous maps from $X \times X$ to $Y$. Thus, if $f^{\prime}$ is differentiable at $u$ and $v, w \in X, f^{\prime \prime}(u)[v, w] \in Y$.

- If $f$ is twice differentiable at every point $u \in U$, we say that $f$ is twice differentiable in $U$. In this case, if the map $f^{\prime \prime}: U \subset X \rightarrow L(X \times X, Y)$ is continuous in $U$, we write $f \in C^{2}(U, Y)$.

Example 1.3.2 (see Ambrosetti and Prodi 1995, Badiale and Serra 2011). Let $X, Y$ and $Z$ be Banach spaces, and $B: X \times Y \rightarrow Z$ a bilinear continuous map. For any pair $(u, v) \in X \times Y$

$$
B(u+h, v+k)-B(u, v)=[B(h, v)+B(u, k)]+B(h, k) .
$$

Since $\|B(h, k)\| \leq C\|h\|\|k\|$, it follows that $\|B(h, k)\|=o(\|(h, k)\|)$ and thus

$$
B^{\prime}(u, v)[h, k]=B(h, v)+B(u, k) .
$$

Now assume that $Y=X$ and that $B$ is symmetric. Denoting

$$
A(u):=B(u, u),
$$

we have, for every $u \in X$,

$$
A^{\prime}(u)[h]=2 B(u, h) .
$$


In this class of mappings we have very important examples:

$$
\begin{aligned}
& A_{1}: L^{2}(\Omega) \rightarrow \mathbb{R}, \quad \text { given by } A_{1}(u):=\int_{\Omega} u^{2} d x \\
& A_{2}: H_{0}^{1}(\Omega) \rightarrow \mathbb{R}, \quad \text { given by } \quad A_{2}(u):=\int_{\Omega}|\nabla u|^{2} d x \\
& A_{3}: H^{1}(\Omega) \rightarrow \mathbb{R}, \quad \text { given by } \quad A_{3}(u):=\int_{\Omega}\left(|\nabla u|^{2}+u^{2}\right) d x,
\end{aligned}
$$

where $\Omega \subset \mathbb{R}^{N}$ is an open set. Thus $A_{1}, A_{2}$ and $A_{3}$ are differentiable at every point and

$$
A_{1}^{\prime}(u)[h]=2 \int_{\Omega} u h d x, \quad A_{2}^{\prime}(u)[h]=2 \int_{\Omega} \nabla u \cdot \nabla h d x \quad \text { and } \quad A_{3}^{\prime}(u)[h]=2 \int_{\Omega}(\nabla u \cdot \nabla h+u h) d x .
$$

Note that $A_{i}(u)$ is the square norm in the corresponding space, and $A_{i}^{\prime}(u)[h]$ is twice the inner product between $u$ and $h$, in the corresponding space. The Sobolev embeddings imply that $A_{1}$ is also differentiable at every point of $H_{0}^{1}(\Omega)$ and of $H^{1}(\Omega)$. In our whole work we use this example, so the differentiability of the functionals will depend on the differentiability of the nonlinear terms.

Among all the differentiation rules, one of the most important still holds for calculus in Banach spaces.

Proposition 1.3.3 (Chain rule). Let

- $X, Y, Z$ Banach spaces

- $U, V$ open sets of $X, Y$, respectively

- $f: U \rightarrow Y, g: V \rightarrow Z$ two maps with $f(U) \subset V$.

If $f$ is differentiable at $u \in U$ and $g$ is differentiable at $v:=f(u)$, then $g \circ f$ is differentiable at $u$ and we have

$$
(g \circ f)^{\prime}(u)[h]=g^{\prime}(v)\left[f^{\prime}(u)[h]\right], \quad \text { for every } h \in X .
$$

A very useful tool in order to find the differential of a map is the Gâteaux differential, the analogous of the directional derivative.

Definition 1.3.4. Let $U \subset X$ be an open set, and $f: U \rightarrow Y$ a map. The function $f$ is Gâteaux differentiable at $x \in U$ in the direction of $h \in X$, if

$$
\lim _{t \rightarrow 0} \frac{f(x+t h)-f(x)}{t}
$$

exists; in that case we write

$$
f_{G}^{\prime}(x)[h]:=\lim _{t \rightarrow 0} \frac{f(x+t h)-f(x)}{t}=\left.\frac{d}{d t} f(x+t h)\right|_{t=0} .
$$

Proposition 1.3.5. Let $f: U \subset X \rightarrow Y$ be a map such that

(i) $f$ is Gâteaux differentiable in $U$ in any direction $h \in X$.

(ii) $f_{G}^{\prime}(u) \in L(X, Y)$, for every $u \in U$.

(iii) $f_{G}^{\prime}: U \rightarrow L(X, Y)$ is continuous at $u$.

Then, $f$ is differentiable at $u$, and $f^{\prime}(u)=f_{G}^{\prime}(u)$. 
By the previous proposition, to calculate the differential of $f$ at $u \in U$, we can find first $f_{G}^{\prime}(u)$. Then, if $f_{G}^{\prime}(u) \in L(X, Y)$ and $f_{G}^{\prime}$ maps continuously $U$ in $L(X, Y)$, we have that $f$ is differentiable at $u$ and $f^{\prime}(u)=f_{G}^{\prime}(u)$. This is a standard procedure.

Now we want to extent the notion of partial derivatives.

Definition 1.3.6. Let $X, Y$ and $Z$ be Banach spaces, and consider the map $f: X \times Y \rightarrow Z$. We define the maps

$$
\begin{array}{lll}
\varphi_{v}:=X \rightarrow Z & \text { by } \quad \varphi_{v}(u):=f(u, v) \\
\varphi_{u}:=Y \rightarrow Z & \text { by } \quad \varphi_{u}(v):=f(u, v)
\end{array}
$$

The partial derivative with respect to $u$ of $f$ at $(u, v)$ is defined by $f_{u}^{\prime}(u, v):=\left(\varphi_{v}\right)^{\prime}(u)$; the partial derivative with respect to $v$ of $f$ at $(u, v)$ is defined by $f_{v}^{\prime}(u, v):=\left(\varphi_{u}\right)^{\prime}(v)$.

Remark 1.3.7. If it is known that $f$ is differentiable at $(u, v)$, then its partial derivatives exist at $(u, v)$ and

$$
f_{u}^{\prime}(u, v)[h]=\left(\varphi_{v}\right)^{\prime}(u)[h]=f^{\prime}(u, v)[h, 0] \quad \text { and } \quad f_{v}^{\prime}(u, v)[k]=\left(\varphi_{u}\right)^{\prime}(v)[k]=f^{\prime}(u, v)[0, k]
$$

For us will be very useful the following (Ambrosetti and Malchiodi 2007, Proposition 1.2, pg. 2)

Proposition 1.3.8. If $f: X \times Y \rightarrow Z$ has the partial derivatives with respect to $u$ and $v$ in a neighborhood $Q \subset X \times Y$ of $(u, v)$ and

$$
\begin{aligned}
& f_{u}^{\prime} \quad \text { maps continuously } Q \text { in } L(X, Z) \\
& f_{v}^{\prime} \quad \text { maps continuously } Q \text { in } L(Y, Z)
\end{aligned}
$$

then $f$ is differentiable at $(u, v)$ and

$$
f^{\prime}(u, v)[h, k]=f_{u}^{\prime}(u, v)[h]+f_{v}^{\prime}(u, v)[k] .
$$

We state the generalization to Banach spaces of the implicit function theorem of calculus in $\mathbb{R}^{n}$; see Ambrosetti and Malchiodi (2007, Theorem 1.4, pg. 3).

Theorem 1.3.9 (Implicit function theorem). Let $T, X$ and $Y$ be Banach spaces; $\Lambda \subset T$, $U \subset X$ open subsets. Assume $f \in C^{1}(\Lambda \times U, Y)$ and let $\left(\lambda_{0}, u_{0}\right) \in \Lambda \times U$ be such that $f\left(\lambda_{0}, u_{0}\right)=0$. If $f_{u}^{\prime}\left(\lambda_{0}, u_{0}\right) \in L(X, Y)$ is invertible, then there are neighborhoods $\Lambda_{0}$ of $\lambda_{0}, U_{0}$ of $u_{0}$ and a map $g \in C^{1}\left(\Lambda_{0}, X\right)$ that satisfy

$$
f(\lambda, u)=0,(\lambda, u) \in \Lambda_{0} \times U_{0} \quad \Leftrightarrow \quad u=g(\lambda), \text { for every } \lambda \in \Lambda_{0} .
$$

The next result is about locally invertible functions. If $f \in C(U, Y), f$ is locally invertible at $u_{0} \in U$ if there are neighborhoods $U_{0}$ of $u_{0}, V_{0}$ of $v_{0}:=f\left(u_{0}\right)$ and a map $g \in C\left(V_{0}, U_{0}\right)$ such that

$$
g(f(u))=u, \forall u \in U_{0}, \quad \text { and } \quad f(g(v))=v, \forall v \in V_{0} .
$$

Notation: $f^{-1}:=g$.

Theorem 1.3.10 (Local inversion theorem). Let $f \in C^{1}(U, Y)$ be a map such that $f^{\prime}\left(u_{0}\right) \in$ $L(X, Y)$ is invertible. Then $f$ is locally invertible at $u_{0}$, with $f^{-1}$ in $C^{1}\left(V_{0}, U_{0}\right)$. Also

$$
\left(f^{-1}\right)^{\prime}(v)=\left(f^{\prime}(u)\right)^{-1}, \quad \forall v \in V_{0}, \quad v:=f(u) .
$$

Now we establish the definition of critical point of a functional. In case of functionals $I$ defined on an open subset of a Banach space $X$ to $\mathbb{R}$, we denote $X^{\prime}:=L(X, \mathbb{R})$. Recall that if $I$ is differentiable at a point $u, I^{\prime}(u) \in X^{\prime}$. 
Definition 1.3.11 (Critical point of a functional). Let $X$ be a Banach space, $U \subset X$ an open set and let $I: U \rightarrow \mathbb{R}$ be a differentiable functional in $U$. We say that $u \in U$ is a critical point of $I$ if

$$
I^{\prime}(u)=0, \quad \text { i.e., } \quad I^{\prime}(u)[v]=0, \text { for every } v \in X \text {. }
$$

Note that if $Y$ is another Banach space, for a functional of two variables $J: Q \subset X \times Y \rightarrow \mathbb{R}$, $Q$ open in $X \times Y$ and $f$ differentiable in $Q$, an element $(u, v) \in Q$ is a critical point of $J$ if

$$
J_{u}^{\prime}(u, v)=0 \quad \text { and } \quad J_{v}^{\prime}(u, v)=0 .
$$

\subsection{The reduction argument for the Schrödinger-Poisson system}

As it was mentioned in the beginning of this chapter, a fact frequently used in this thesis consists in reducing the Schrödinger-Poisson system to a single equation. In this section we give the key procedure that let us solve a Schrödinger-Poisson system using critical point theory for a functional that depends only on one variable. A similar reduction argument can be employed to deal with the system appearing in Chapter 2. In the literature it is already standard, and it was first introduced in Benci and Fortunato (1998). Specifically, we consider the system

$$
\begin{cases}-\Delta u+u+\phi u=|u|^{p-2} u & \text { in } \mathbb{R}^{3} \\ -\Delta \phi=u^{2} & \text { in } \mathbb{R}^{3}\end{cases}
$$

with $2<p<6$. For a weak solution (from now on in this chapter, we just simply say solution) of the previous system we understand a pair $(u, \phi) \in H^{1}\left(\mathbb{R}^{3}\right) \times D^{1,2}\left(\mathbb{R}^{3}\right)$ that satisfies

$$
\begin{aligned}
\int_{\mathbb{R}^{3}}[\nabla u \cdot \nabla v+(1+\phi) u v] d x & =\int_{\mathbb{R}^{3}}|u|^{p-2} u v d x, \\
\int_{\mathbb{R}^{3}} \nabla \phi \cdot \nabla \psi d x & =\int_{\mathbb{R}^{3}} u^{2} \psi d x,
\end{aligned}
$$

for every pair $(v, \psi) \in H^{1}\left(\mathbb{R}^{3}\right) \times D^{1,2}\left(\mathbb{R}^{3}\right)$. Let us define the functional $F: H^{1}\left(\mathbb{R}^{3}\right) \times D^{1,2}\left(\mathbb{R}^{3}\right) \rightarrow \mathbb{R}$ by

$$
F(u, \phi):=\frac{1}{2} \int_{\mathbb{R}^{3}}\left[|\nabla u|^{2}+(1+\phi) u^{2}\right] d x-\frac{1}{p} \int_{\mathbb{R}^{3}}|u|^{p} d x-\frac{1}{4} \int_{\mathbb{R}^{3}}|\nabla \phi|^{2} d x .
$$

Proposition 1.4.1. The functional $F$ is $C^{1}$ and its critical points are the solutions of (1.4.1).

Proof. Consider the functional

$$
L(u, \phi):=\frac{1}{2} \int_{\mathbb{R}^{3}} \phi u^{2} d x .
$$

First note that the map $L(u, \cdot): D^{1,2}\left(\mathbb{R}^{3}\right) \rightarrow \mathbb{R}$ is linear, and it is continuous because

$$
\int_{\mathbb{R}^{3}} \phi u^{2} d x \leq|\phi|_{6}\left|u^{2}\right|_{6 / 5}=|\phi|_{6}|u|_{12 / 5}^{2} \leq C\|u\|^{2}\|\phi\|_{D}=C_{u}\|\phi\|_{D}
$$

Therefore

$$
L_{\phi}(u, \phi)[\psi]=L(u, \psi)=\frac{1}{2} \int_{\mathbb{R}^{3}} \psi u^{2} d x
$$

On the other hand

$$
\left(L_{u}\right)_{G}(u, \phi)[v]:=\left.\frac{d}{d t} L(u+t v, \phi)\right|_{t=0}=\int_{\mathbb{R}^{3}} \phi u v d x
$$

and since

$$
\int_{\mathbb{R}^{3}} \phi u v d x \leq|\phi|_{6}|u|_{12 / 5}|v|_{12 / 5} \leq C\|u\|\|v\|\|\phi\|_{D}
$$


it follows that

$$
\left(L_{u}\right)_{G}(u, \phi) \in H^{-1} \quad \text { and } \quad\left(L_{u}\right)_{G}(\cdot, \phi): H^{1}\left(\mathbb{R}^{3}\right) \rightarrow H^{-1} \text { is continuous. }
$$

Hence $L_{u}$ exists at $(u, \phi)$ and

$$
L_{u}(u, \phi)=\left(L_{u}\right)_{G}(u, \phi) .
$$

To sum up, $L$ has partial derivatives with respect to $u$ and $\phi$ at any point $(u, \phi)$. Now we want to see that the maps

$$
L_{u}: H^{1}\left(\mathbb{R}^{3}\right) \times D^{1,2}\left(\mathbb{R}^{3}\right) \rightarrow H^{-1} \quad \text { and } \quad L_{\phi}: H^{1}\left(\mathbb{R}^{3}\right) \times D^{1,2}\left(\mathbb{R}^{3}\right) \rightarrow D^{\prime}
$$

are continuous. Assume that $\left(u_{n}, \phi_{n}\right) \rightarrow(u, \phi)$ in $H^{1}\left(\mathbb{R}^{3}\right) \times D^{1,2}\left(\mathbb{R}^{3}\right)$. For the continuity of $L_{u}$, note that if $v \in H^{1}\left(\mathbb{R}^{3}\right)$ with $\|v\| \leq 1$,

$$
\left|\left(L_{u}\left(u_{n}, \phi_{n}\right)-L_{u}(u, \phi)\right)[v]\right| \leq \int_{\mathbb{R}^{3}}\left|\left(\phi_{n} u_{n}-\phi u\right) v\right| d x \leq \int_{\mathbb{R}^{3}}\left|\phi_{n}-\phi\right|\left|u_{n}\right||v| d x+\int_{\mathbb{R}^{3}}|\phi|\left|u_{n}-u\right||v| d x .
$$

Using the Hölder inequality and the Sobolev embeddings

$$
\int_{\mathbb{R}^{3}}|\phi|\left|u_{n}-u\right||v| d x \leq|\phi| 6\left|u_{n}-u\right|_{12 / 5}|v|_{12 / 5} \leq C\|\phi\|_{D}\left\|u_{n}-u\right\|\|v\| \leq C\left\|u_{n}-u\right\| .
$$

On the other hand, since $\left\{u_{n}\right\}$ is bounded in $H^{1}\left(\mathbb{R}^{3}\right)$

$$
\int_{\mathbb{R}^{3}}\left|\phi_{n}-\phi\right|\left|u_{n}\right||v| d x \leq\left|\phi_{n}-\phi\right|_{6}\left|u_{n}\right|_{12 / 5}|v|_{12 / 5} \leq C\left\|\phi_{n}-\phi\right\|_{D}\left\|u_{n}\right\|\|v\| \leq C\left\|\phi_{n}-\phi\right\|_{D} .
$$

Therefore

$$
\left\|L_{u}\left(u_{n}, \phi_{n}\right)-L_{u}(u, \phi)\right\|_{\star} \leq C\left(\left\|\phi_{n}-\phi\right\|_{D}+\left\|u_{n}-u\right\|\right)=o_{n}(1) .
$$

For the continuity of $L_{\phi}$, taking an element $\psi \in D^{1,2}\left(\mathbb{R}^{3}\right)$ with $\|\psi\|_{D} \leq 1$,

$$
\begin{aligned}
\left|\left(L_{\phi}\left(u_{n}, \phi_{n}\right)-L_{\phi}(u, \phi)\right)[\psi]\right| \leq \int_{\mathbb{R}^{3}}|\psi| \mid & u_{n}^{2}-u^{2} \mid d x \\
& \leq\left|u_{n}^{2}-u^{2}\right|_{6 / 5}|\psi|_{6} \leq C\left|u_{n}^{2}-u^{2}\right|_{6 / 5}\|\psi\|_{D} \leq C\left|u_{n}^{2}-u^{2}\right|_{6 / 5} .
\end{aligned}
$$

Since $u_{n} \rightarrow u$ in $H^{1}\left(\mathbb{R}^{3}\right)$, up to a subsequence

$$
\begin{array}{lll}
u_{n} \rightarrow u \text { in } L^{12 / 5}\left(\mathbb{R}^{3}\right) & \Rightarrow & u_{n}(x) \rightarrow u(x) \text { a.e. } x \in \mathbb{R}^{3} \\
u_{n} \rightarrow u \text { in } L^{12 / 5}\left(\mathbb{R}^{3}\right) & \Rightarrow & \left|u_{n}^{2}\right|_{6 / 5}=\left|u_{n}\right|_{12 / 5}^{2} \rightarrow|u|_{12 / 5}^{2}=\left|u^{2}\right|_{6 / 5},
\end{array}
$$

and from Brezis-Lieb lemma $u_{n}^{2} \rightarrow u^{2}$ in $L^{6 / 5}\left(\mathbb{R}^{3}\right)$. Thus

$$
\left\|L_{\phi}\left(u_{n}, \phi_{n}\right)-L_{\phi}(u, \phi)\right\|_{\star}=o_{n}(1)
$$

Because of (1.4.4), (1.4.5) and Proposition 1.3.8, $L$ is $C^{1}$. The fact that the other terms of $F$ are $C^{1}$ is a consequence of the differentiability of the norm (already exposed in this chapter, see Example 1.3.2) and from the Sobolev embeddings. Indeed, for the functional

$$
K(u):=\frac{1}{p} \int_{\mathbb{R}^{3}}|u|^{p} d x,
$$

it is easy to see that

$$
K_{G}^{\prime}(u)[v]=\int_{\mathbb{R}^{3}}|u|^{p-2} u v d x,
$$


and from here it is clear that $K_{G}^{\prime}(u) \in H^{-1}$. Now, if $u_{n} \rightarrow u$ in $H^{1}\left(\mathbb{R}^{3}\right)$, it can be shown that

$$
\int_{\mathbb{R}^{3}}\left|u_{n}\right|^{p-2} u_{n} v d x \rightarrow \int_{\mathbb{R}^{3}}|u|^{p-2} u v d x, \quad \forall v \in H^{1}\left(\mathbb{R}^{3}\right),
$$

arguing as in the proof of Willem (1996, Lemma 8, pg. 118).

For $v \in H^{1}\left(\mathbb{R}^{3}\right), \psi \in D^{1,2}\left(\mathbb{R}^{3}\right)$ the partial derivatives of $F$ are given by

$$
\begin{aligned}
F_{u}^{\prime}(u, \phi)[v] & =\int_{\mathbb{R}^{3}}\left[\nabla u \cdot \nabla v+(1+\phi) u v-|u|^{p-2} u v\right] d x, \\
F_{\phi}^{\prime}(u, \phi)[\psi] & =\frac{1}{2} \int_{\mathbb{R}^{3}}\left(\psi u^{2}-\nabla \phi \cdot \nabla \psi\right) d x .
\end{aligned}
$$

Finally,

$(u, \phi)$ is a critical point of $F \Leftrightarrow F_{u}^{\prime}(u, \phi)=0, F_{\phi}^{\prime}(u, \phi)=0 \Leftrightarrow(u, \phi)$ is a weak solution of (1.4.1), and we conclude.

Note that $F$ is neither bounded from below nor from above. Indeed, when $t \rightarrow \infty$

$$
F(t u, \phi) \leq \frac{t^{2}}{2} \int_{\mathbb{R}^{3}}\left[|\nabla u|^{2}+(1+\phi) u^{2}\right] d x-\frac{t^{p}}{p} \int_{\mathbb{R}^{3}}|u|^{p} d x \rightarrow-\infty,
$$

whereas, if we consider the function $u_{k} \in H^{1}\left(\mathbb{R}^{3}\right)$ for $k \in \mathbb{N}$ defined by (see Rabinowitz 1986, pg. 2)

$$
u_{k}(x):= \begin{cases}\sin k|x|, & 0 \leq|x| \leq \pi \\ 0, & |x|>\pi,\end{cases}
$$

we have

$$
\begin{aligned}
F\left(u_{k}, 0\right) & =\frac{1}{2} \int_{\mathbb{R}^{3}}\left(\left|\nabla u_{k}\right|^{2}+u_{k}^{2}\right) d x-\frac{1}{p} \int_{\mathbb{R}^{3}}\left|u_{k}\right|^{p} d x \\
& \geq \frac{1}{2} \int_{\mathbb{R}^{3}}\left|\nabla u_{k}\right|^{2} d x-\frac{1}{p} \int_{\mathbb{R}^{3}}\left|u_{k}\right|^{p} d x \\
& =\pi k^{2}\left[\int_{0}^{\pi} r^{2} d r+\int_{0}^{\pi} r^{2} \cos 2 k r d r\right]-\frac{4 \pi}{p} \int_{0}^{\pi} r^{2}|\sin k r|^{p} d r \\
& \geq \pi^{2} k^{2}\left(\frac{\pi^{2}}{3}+\frac{1}{2 k^{2}}\right)-\frac{4 \pi^{4}}{3 p} \rightarrow \infty,
\end{aligned}
$$

when $k \rightarrow \infty$. Fortunately, the substitution method of Benci and Fortunato (1998) let us apply the critical point theory. We want to remark here that in this section we are not interested in solving the system (1.4.1), but to find a functional that depends of one variable, whose critical points are the solutions of (1.4.1). With this purpose in mind, we proceed. First we use the following

Proposition 1.4.2. Given $u \in H^{1}\left(\mathbb{R}^{3}\right)$, there is a unique solution $\phi_{u} \in D^{1,2}\left(\mathbb{R}^{3}\right)$ for the problem

$$
-\Delta \phi=u^{2} \text { in } \mathbb{R}^{3},
$$

so that the map $\Phi: H^{1}\left(\mathbb{R}^{3}\right) \rightarrow D^{1,2}\left(\mathbb{R}^{3}\right)$ given by $\Phi[u]:=\phi_{u}$ is well defined and its graph is

$$
G_{\Phi}=\left\{(u, \phi) \in H^{1}\left(\mathbb{R}^{3}\right) \times D^{1,2}\left(\mathbb{R}^{3}\right): F_{\phi}^{\prime}(u, \phi)=0\right\} .
$$

Proof. Let $u \in H^{1}\left(\mathbb{R}^{3}\right)$ be a fixed element; consider the linear transformation $T_{u}: D^{1,2}\left(\mathbb{R}^{3}\right) \rightarrow \mathbb{R}$ given by

$$
T_{u}[\psi]:=\int_{\mathbb{R}^{3}} \psi u^{2} d x
$$


that it is also continuous because of (1.4.2); thus $T \in D^{\prime}$. By Riesz representation theorem, there is a unique element $\phi_{u} \in D^{1,2}\left(\mathbb{R}^{3}\right)$ such that

$$
\int_{\mathbb{R}^{3}} \nabla \phi_{u} \cdot \nabla \psi d x=T[\psi]=\int_{\mathbb{R}^{3}} \psi u^{2} d x, \text { for every } \psi \in D^{1,2}\left(\mathbb{R}^{3}\right),
$$

i.e. $\phi_{u}$ is the only solution of (1.4.6). If $G_{\Phi}$ denotes the graph of $\Phi$, given $(u, \phi) \in H^{1}\left(\mathbb{R}^{3}\right) \times D^{1,2}\left(\mathbb{R}^{3}\right)$

$$
F_{\phi}^{\prime}(u, \phi)=0 \Leftrightarrow \int_{\mathbb{R}^{3}} \nabla \phi \cdot \nabla \psi d x=\int_{\mathbb{R}^{3}} \psi u^{2} d x \Leftrightarrow \phi \text { is a solution of }(1.4 .6) \Leftrightarrow \phi=\phi_{u}=\Phi[u] .
$$

Remark 1.4.3. Recall that from (1.4.2) we have the inequality

$$
\int_{\mathbb{R}^{3}} \psi u^{2} d x \leq C\|u\|^{2}\|\psi\|_{D}, \text { for every } \psi \in D^{1,2}\left(\mathbb{R}^{3}\right) .
$$

Also by (1.4.8), the solution $\phi_{u}$ of (1.4.6) satisfies

$$
\int_{\mathbb{R}^{3}} \nabla \phi_{u} \cdot \nabla \psi d x=\int_{\mathbb{R}^{3}} \psi u^{2} d x, \text { for every } \psi \in D^{1,2}\left(\mathbb{R}^{3}\right) .
$$

Therefore, taking $\psi=\phi_{u}$ we can write

$$
\left\|\phi_{u}\right\|_{D}^{2}=\int_{\mathbb{R}^{3}}\left|\nabla \phi_{u}\right|^{2} d x=\int_{\mathbb{R}^{3}} \phi_{u} u^{2} d x \leq C\|u\|^{2}\left\|\phi_{u}\right\|_{D},
$$

from which

$$
\left\|\phi_{u}\right\|_{D} \leq C\|u\|^{2}
$$

Thus, we obtain

$$
\int_{\mathbb{R}^{3}} \phi_{u} u^{2} d x \leq C\|u\|^{4} .
$$

The last inequality, in the adapted version in the following chapters, will be very useful.

When we work in the whole space, as it is our present case, we can write a simple, explicit formula for $\phi_{u}$. In fact, it can be shown that $1 /(4 \pi|\cdot|)$ is the fundamental solution (see the details about fundamental solutions, for instance, in Kesavan 1989, Section 1.7, pg. 29) to $-\Delta$ in $\mathbb{R}^{3}$, i.e.,

$$
-\Delta\left(\frac{1}{4 \pi|\cdot|}\right)=\delta
$$

where $\delta$ denotes the usual Dirac distribution. Then

$$
-\Delta\left(\frac{1}{4 \pi|\cdot|} \star u^{2}\right)=u^{2} \star \delta=u^{2}
$$

implying that

$$
\phi_{u}(x)=\left(\frac{1}{4 \pi|\cdot|} \star u^{2}\right)(x)=\frac{1}{4 \pi} \int_{\mathbb{R}^{3}} \frac{u^{2}(y)}{|x-y|} d y, \forall x \in \mathbb{R}^{3} .
$$

There is also an expression for the solution to the equation in a ball $B_{R}$. However, a corrector function will be necessary in this situation. In this thesis we consider only the explicit formula for $\phi_{u}$ when the domain is $\mathbb{R}^{N}$.

Proposition 1.4.4.

$$
\Phi \in C^{1}\left(H^{1}\left(\mathbb{R}^{3}\right), D^{1,2}\left(\mathbb{R}^{3}\right)\right) .
$$


Proof. We define the map $J: H^{1}\left(\mathbb{R}^{3}\right) \times D^{1,2}\left(\mathbb{R}^{3}\right) \rightarrow D^{\prime}$ by $J(u, \phi):=F_{\phi}^{\prime}(u, \phi)$ and we claim that

$$
J \in C^{1}\left(H^{1}\left(\mathbb{R}^{3}\right) \times D^{1,2}\left(\mathbb{R}^{3}\right), D^{\prime}\right)
$$

In fact, since

$$
J(u, \phi)[\cdot]=L(u, \cdot)-\frac{1}{2} \int_{\mathbb{R}^{3}} \nabla \phi \cdot \nabla(\cdot) d x,
$$

where $L$ is the $C^{1}$ map used in the proof of the Proposition 1.4.1, (1.4.10) is immediate. Thus we can write

$$
\begin{aligned}
& \left(J_{u}(u, \phi)[v]\right)[\psi]=F_{u \phi}^{\prime \prime}(u, \phi)[v, \psi]=\int_{\mathbb{R}^{3}} u v \psi d x \\
& \left(J_{\phi}(u, \phi)[\nu]\right)[\psi]=F_{\phi \phi}^{\prime \prime}(u, \phi)[\nu, \psi]=-\frac{1}{2} \int_{\mathbb{R}^{3}} \nabla \nu \cdot \nabla \psi d x .
\end{aligned}
$$

Now we show that

$$
J_{\phi}^{\prime}(u, \phi) \in L\left(D^{1,2}\left(\mathbb{R}^{3}\right), D^{\prime}\right) \text { is invertible. }
$$

Indeed, since

$$
J_{\phi}(u, \phi)[\nu]=F_{\phi \phi}^{\prime \prime}(u, \phi)[\nu, \cdot]=-\frac{1}{2} \int_{\mathbb{R}^{3}} \nabla \nu \cdot \nabla(\cdot) d x,
$$

up to a constant factor, $J_{\phi}(u, \phi)$ is the Riesz isomorphism between $D^{1,2}\left(\mathbb{R}^{3}\right)$ and its dual, $D^{\prime}$. Finally, let $u_{0} \in H^{1}\left(\mathbb{R}^{3}\right)$ be fixed, but arbitrary. Because of (1.4.7),

$$
\left(u_{0}, \Phi\left[u_{0}\right]\right) \in G_{\Phi} \quad \Rightarrow \quad J\left(u_{0}, \Phi\left[u_{0}\right]\right)=0 .
$$

Since (1.4.10) holds true and $J_{\phi}^{\prime}\left(u_{0}, \Phi\left[u_{0}\right]\right) \in L\left(D^{1,2}\left(\mathbb{R}^{3}\right), D^{\prime}\right)$ is invertible by (1.4.11), in virtue of the implicit theorem function there exist neighborhoods $U_{0}$ of $u_{0}, V_{0}$ of $\Phi\left[u_{0}\right]$ and a map $g \in$ $C^{1}\left(U_{0}, D^{1,2}\left(\mathbb{R}^{3}\right)\right)$ such that

$$
J(u, \phi)=0 \text { and }(u, \phi) \in U_{0} \times V_{0} \quad \Leftrightarrow \quad \phi=g(u), \forall u \in U_{0} .
$$

But then, for every $u \in U_{0}$,

$$
J(u, g(u))=0 \quad \Rightarrow \quad(u, g(u)) \in G_{\Phi},
$$

from which

$$
g=\left.\Phi\right|_{U_{0}}
$$

In particular $\left.\Phi\right|_{U_{0}} \in C^{1}\left(U_{0}, D^{1,2}\left(\mathbb{R}^{3}\right)\right)$, and by the arbitrariness of $u_{0}$ we conclude.

Let us define the functional $I: H^{1}\left(\mathbb{R}^{3}\right) \rightarrow \mathbb{R}$ by $I(u):=F(u, \Phi[u])$. To obtain the explicit form of $I$, note that

$$
\begin{aligned}
I(u) & =F\left(u, \phi_{u}\right) \\
& =\frac{1}{2} \int_{\mathbb{R}^{3}}\left[|\nabla u|^{2}+\left(1+\phi_{u}\right) u^{2}\right] d x-\frac{1}{p} \int_{\mathbb{R}^{3}}|u|^{p} d x-\frac{1}{4} \int_{\mathbb{R}^{3}}\left|\nabla \phi_{u}\right|^{2} d x \\
& =\frac{1}{2} \int_{\mathbb{R}^{3}}\left(|\nabla u|^{2}+u^{2}\right) d x+\frac{1}{4} \int_{\mathbb{R}^{3}} \phi_{u} u^{2} d x-\frac{1}{p} \int_{\mathbb{R}^{3}}|u|^{p} d x,
\end{aligned}
$$

where we have used the relation (see (1.4.9))

$$
\int_{\mathbb{R}^{3}}\left|\nabla \phi_{u}\right|^{2} d x=\int_{\mathbb{R}^{3}} \phi_{u} u^{2} d x .
$$

Since $F \in C^{1}\left(H^{1}\left(\mathbb{R}^{3}\right) \times D^{1,2}\left(\mathbb{R}^{3}\right), \mathbb{R}\right)$ and $\Phi \in C^{1}\left(H^{1}\left(\mathbb{R}^{3}\right), D^{1,2}\left(\mathbb{R}^{3}\right)\right), I$ is a $C^{1}$ functional in $H^{1}\left(\mathbb{R}^{3}\right)$. 
Furthermore, because of (1.4.7)

$$
\begin{aligned}
I^{\prime}(u) & =F_{u}^{\prime}(u, \Phi[u])+F_{\phi}^{\prime}(u, \Phi[u]) \Phi^{\prime}[u] \\
& =F_{u}^{\prime}(u, \Phi[u]) .
\end{aligned}
$$

Proposition 1.4.5. For $(u, \phi) \in H^{1}\left(\mathbb{R}^{3}\right) \times D^{1,2}\left(\mathbb{R}^{3}\right)$,

$$
(u, \phi) \text { is a critical point of } F \Leftrightarrow u \text { is a critical point of } I \text { and } \phi=\Phi[u]
$$

Proof.

$$
\begin{aligned}
(u, \phi) \text { is a critical point of } F & \Leftrightarrow F_{u}^{\prime}(u, \phi)=0 \text { and } F_{\phi}^{\prime}(u, \phi)=0 \\
& \Leftrightarrow F_{u}^{\prime}(u, \phi)+F_{\phi}^{\prime}(u, \phi) \Phi^{\prime}[u]=0 \text { and } \phi=\Phi[u] \\
& \Leftrightarrow I^{\prime}(u)=0 \text { and } \phi=\Phi[u] \\
& \Leftrightarrow u \text { is a critical point of } I \text { and } \phi=\Phi[u] .
\end{aligned}
$$

Thus the system (1.4.1) has been reduced to the equation

$$
-\Delta u+u+\Phi[u] u=|u|^{p-2} u \text { in } \mathbb{R}^{3},
$$

whose solutions are the critical points of $I$.

\subsection{A brief summary of the theory of Hilbert manifolds}

Here we introduce some basic concepts about Hilbert manifolds that are going to be used in Chapter 2, where we intend to find critical points of a $C^{1}$ functional constrained to a suitable manifold. Our exposition is based on the "outline" explained in Ambrosetti and Malchiodi (2007, Chapter 6, pg. 89).

Before the definition of a Hilbert manifold, we give the notion of diffeomorphism that can be formulated in the more general context of Banach spaces.

Definition 1.5.1. Let $X, Y$ be Banach spaces, $U \subset X, V \subset Y$ open subsets in $X, Y$ respectively. A map $f: U \rightarrow V$ is a $C^{1}$ diffeomorphism if $f$ is $C^{1}$ differentiable, bijective, and its inverse $f^{-1}$ is also $C^{1}$ differentiable.

In this section, occasionally, we are going to use the following notation: If $X, Y$ are Banach spaces, $U \subset X$ is an open subset of $X$ and $f: U \rightarrow Y$ is differentiable at $u \in U$, then $d f(u):=$ $f^{\prime}(u) \in L(X, Y)$.

Definition 1.5.2. Let $X$ be a Hilbert space and $\mathcal{M}$ a topological space. We say that $\mathcal{M}$ is a $C^{1}$ Hilbert manifold modelled on $X$ if it can be covered with an indexed family of open sets in $\mathcal{M}$ $\left\{U_{i}\right\}_{i \in \mathcal{I}}$ such that

(i) for each $i \in \mathcal{I}$, there is a map $\psi_{i}: U_{i} \rightarrow X$ such that $V_{i}:=\psi_{i}\left(U_{i}\right)$ is an open set in $X$; $\psi_{i}$ is a homeomorphism onto its image $V_{i}$,

(ii) if there are two pairs $\left(U_{i}, \psi_{i}\right),\left(U_{j}, \psi_{j}\right)$ with $U_{i} \cap U_{j} \neq \emptyset$, then the map $\psi_{j} \circ \psi_{i}^{-1}: \psi_{i}\left(U_{i} \cap U_{j}\right) \rightarrow$ $\psi_{j}\left(U_{i} \cap U_{j}\right)$ is $C^{1}$ differentiable.

We say that the pair $\left(U_{i}, \psi_{i}\right)$ is a chart of the manifold, the maps $\psi_{j} \circ \psi_{i}^{-1}$ are changes of charts, and the pair $\left(V_{i}, \psi_{i}^{-1}\right)$ is a local parametrization of $\mathcal{M}$.

The Hilbert manifolds that are considered here fall in the following situation: Given a Hilbert space $E, \mathcal{M} \subset E$ has the subspace topology induced by the topology of $E$, and $X \subset E$ is a Hilbert subspace of $E$. 
Remark 1.5.3. Henceforth, we assume that for every point $p \in \mathcal{M}$, there are $\widetilde{U}, \widetilde{V}$ open subsets of $E$ and $\widetilde{\varphi}: \widetilde{V} \rightarrow \widetilde{U}$ a $C^{1}$ diffeomorphism such that

- $p \in \widetilde{U}$,

- defining $U:=\widetilde{U} \cap \mathcal{M}, V:=\widetilde{V} \cap X$ and $\varphi:=\left.\widetilde{\varphi}\right|_{V}$, we have $x:=\varphi^{-1}(p) \in V$ and $U=\varphi(V)$.

Thus the pair $(V, \varphi)$ is a local parametrization of $\mathcal{M}$.

Definition 1.5.4. Given $p \in \mathcal{M}$, the tangent space to $\mathcal{M}$ at $p$ is defined by

$$
T_{p} \mathcal{M}:=d \widetilde{\varphi}(x)[X] .
$$

Recall that $d \widetilde{\varphi}(x) \in L(E, E)$.

With respect to the previous definition, the following facts can be shown (see the details in Ambrosetti and Malchiodi 2007, pg. 90-93):

(i) $T_{p} \mathcal{M}$ is independent of the local parametrization that is chosen.

(ii) $T_{p} \mathcal{M}$ is a Hilbert space homeomorphic to $X$.

(iii) $T_{p} \mathcal{M}$ is the set of tangent vectors at $p$ to $C^{1}$ curves on $\mathcal{M}$.

Using the inner product on $E$, it is possible to define an inner product on each tangent space $T_{p} \mathcal{M}$ that allows us to calculate the length of a smooth curve on $\mathcal{M}$. Under the additional assumption of arcwise connectivity on $\mathcal{M}$, it can be considered as a metric space.

Remark 1.5.5. From now on, when we take into account a Hilber manifold $\mathcal{M}$, it will be understood that $\mathcal{M}$ is arcwise connected and a complete metric space.

Now we explore the notion of differentiable map between manifolds. Let $\mathcal{M}_{i} \subset E_{i}$ be manifolds modelled on the Hilbert spaces $X_{i} \subset E_{i}$, respectively, $i=1,2$. Suppose that there exist an open subset $U_{1} \subset E_{1}$ and a $C^{1}$ map $\tilde{f}: U_{1} \rightarrow E_{2}$ such that

$$
\mathcal{M}_{1} \subset U_{1} \text { and } f=\left.\tilde{f}\right|_{\mathcal{M}_{1}} .
$$

Definition 1.5.6. The differential of $f: \mathcal{M}_{1} \rightarrow \mathcal{M}_{2}$ at $p$ is defined by

$$
d_{\mathcal{M}_{1}} f(p):=\left.d \widetilde{f}(p)\right|_{T_{p} \mathcal{M}_{1}} .
$$

Note that $d \widetilde{f}(p) \in L\left(E_{1}, E_{2}\right)$.

The definition is consistent because it is independent of the map $\widetilde{f}$.

Example 1.5.7. Let $E$ be a Hilbert space and let $I: U \subset E \rightarrow \mathbb{R}$ be a $C^{1}$ functional, with $U$ an open subset of $E$ that contains a manifold $\mathcal{M}$ modelled on the Hilbert subspace $X$ of $E$. Then for a point $p \in \mathcal{M}$,

$$
d_{\mathcal{M}} I(p)=\left.d I(p)\right|_{T_{p} \mathcal{M}} .
$$

Let us denote by $(\cdot, \cdot)$ the inner product on $E$. Since $d I(p) \in E^{\prime}$, by Riesz representation theorem, there exists a unique $\nabla I(p) \in E$ such that

$$
(\nabla I(p), v)=d I(p)[v], \quad \forall v \in E .
$$

$\nabla I(p)$ is called the gradient of $I$ at $p$. Similarly we can define the constrained gradient of $I$ at $p$ on account of the Riesz representation theorem (recall that $T_{p} \mathcal{M}$ is also a Hilbert space),

$$
\left(\nabla_{\mathcal{M}} I(p), v\right)=d_{\mathcal{M}} I(p)[v], \quad \forall v \in T_{p} \mathcal{M}
$$


Thus, by using (1.5.1)

$$
\left(\nabla_{\mathcal{M}} I(p), v\right)=(\nabla I(p), v) \quad \forall v \in T_{p} \mathcal{M} .
$$

Therefore, the constraint gradient can be considered as the projection of the (free) gradient on the tangent space $T_{p} \mathcal{M}$.

The last example is particularly interesting for our work.

Definition 1.5.8. Suppose that $I: E \rightarrow \mathbb{R}$ is a $C^{1}$ functional and $\mathcal{M}$ is a $C^{1}$ manifold modelled on $X \subset E$. The point $z \in \mathcal{M}$ is a constraint critical point of $I$ on $\mathcal{M}$ if

$$
d_{\mathcal{M}} I(z)=0, \quad \text { i.e., } \quad d_{\mathcal{M}} I(z)[v]=0, \forall v \in T_{z} \mathcal{M} .
$$

Thus from (1.5.2) and (1.5.3),

$$
z \text { constrained critical point of } I \text { on } \mathcal{M} \Rightarrow(\nabla I(z), v)=0, \forall v \in T_{z} \mathcal{M} \text {, }
$$

that is to say, if $z$ is constrained critical point of $I$ on $\mathcal{M}$, then $\nabla I(z)$ is orthogonal to $T_{z} \mathcal{M}$.

Example 1.5.9. Assume that $E, \mathcal{M}, X$ and $I \in C^{1}(E, \mathbb{R})$ are as in the previous definition. An element $z \in \mathcal{M}$ is a local constrained minimum of $I$ on $\mathcal{M}$ if there is a neighborhood $\Theta$ of $z$ in $E$ for which

$$
I(z) \leq I(u), \quad \forall u \in \Theta \cap \mathcal{M} .
$$

Let $(V, \varphi)$ be a local parametrization of $\mathcal{M}$ such that $0 \in V$ and $z=\varphi(0)$. In terms of the local parametrization, the fact that $z$ is a local constrained minimum means that, for some $W \subset X$ neighborhood of 0 ,

$$
I(\varphi(0)) \leq I(\varphi(w)) \quad \forall w \in W .
$$

Therefore, $z$ is a local constrained minimum of $I$ on $\mathcal{M}$ if and only if $0 \in X$ is a local minimum of $I \circ \varphi$. Hence

$$
0 \text { is a critical point of the functional } I \circ \varphi: W \subset X \rightarrow \mathbb{R} \text {. }
$$

Using the chain rule, the last statement can be written as

$$
d I(z)[d \varphi(0)[w]]=d(I \circ \varphi)(0)[w]=0, \forall w \in X .
$$

From $\varphi=\left.\widetilde{\varphi}\right|_{V}$ we have

$$
d \varphi(0)[X]=d \widetilde{\varphi}(0)[X]=T_{z} \mathcal{M},
$$

so that

$$
d I(z)[d \varphi(0)[w]]=0, \forall w \in X \quad \Leftrightarrow \quad d I(z)[v]=0, \forall v \in T_{z} \mathcal{M} .
$$

In conclusion, if $z$ is a local constrained minimum of $I$ on $\mathcal{M}$, then $z$ is a constrained critical point of $I$ on $\mathcal{M}$.

When $\mathcal{M} \subset E$ is modelled on a subspace $X \subset E$ of codimension one, $\mathcal{M}$ is denominated a manifold of codimension one. This is the case when we consider

$$
\mathcal{M}:=J^{-1}(0), \quad \text { where } J \in C^{1}(E, \mathbb{R}), \quad \text { and } \nabla J(u) \neq 0, \forall u \in \mathcal{M} .
$$

Indeed, for $p \in \mathcal{M}$, we define

$$
X_{p}:=\{v \in E:(\nabla J(p), v)=0\} .
$$

Recall that $(\cdot, \cdot)$ is the inner product in $E . X_{p}$ is a linear subspace of $E$, and we have the decomposition $E=X_{p} \oplus\langle w\rangle$, where

$$
w:=\frac{\nabla J(p)}{\|\nabla J(p)\|^{2}}
$$


Thus $X_{p}$ is a subspace of codimension one. Now we are going to show that $\mathcal{M}$ is a manifold modelled on $X_{p}$. Let $\psi: E \rightarrow E$ be the map given by

$$
\psi(u):=u-p-(\nabla J(p), u-p) w+J(u) w .
$$

Taking the inner product with $\nabla J(p)$ and using the fact that

$$
(\nabla J(p), w)=\frac{(\nabla J(p), \nabla J(p))}{\|\nabla J(p)\|^{2}}=1,
$$

we have

$$
\begin{aligned}
(\nabla J(p), \psi(u)) & =(\nabla J(p), u-p)-(\nabla J(p), u-p)(\nabla J(p), w)+J(u)(\nabla J(p), w) \\
& =(\nabla J(p), u-p)-(\nabla J(p), u-p)+J(u) \\
& =J(u),
\end{aligned}
$$

from which

$$
\begin{aligned}
\psi(u) \in X_{p} & \Leftrightarrow \quad(\nabla J(p), \psi(u))=0 \\
& \Leftrightarrow \quad J(u)=0 \\
& \Leftrightarrow u \in \mathcal{M} .
\end{aligned}
$$

Note that $\psi(p)=0$ and that $\psi$ is clearly differentiable. Concretely,

$$
\begin{aligned}
d \psi(u)[v] & =v-(\nabla J(p), v) w+(d J(u)[v]) w \\
& =v-(\nabla J(p), v) w+(\nabla J(u), v) w,
\end{aligned}
$$

and thus

$$
d \psi(p)[v]=v-(\nabla J(p), v) w+(\nabla J(p), v) w=v=i d[v] .
$$

Therefore, by the local inversion theorem, there are $\widetilde{U} \subset E$ neighborhood of $p$ and $\widetilde{V} \subset E$ neighborhood of 0 such that $\psi$ is a diffeomorphism between them; denote $\widetilde{\varphi}:=\psi^{-1}$. Defining

$$
U:=\widetilde{U} \cap \mathcal{M}, \quad V:=\widetilde{V} \cap X_{p}, \quad \varphi:=\left.\widetilde{\varphi}\right|_{V}
$$

it is true that

$$
0=\varphi^{-1}(p), \quad \text { and } \quad \varphi(V)=U .
$$

Since all the subspaces $X_{p}$ can be made isomorphic to a common $X \subset E$ Hilbert subspace of codimension one, from the last argument we can claim that $\mathcal{M}$ is a manifold of codimension one modelled on $X$, with local parametrization $(V, \varphi)$ at $p$. Furthermore

$$
d \varphi(0)=d\left(\psi^{-1}\right)(0)=(d \psi(p))^{-1}=i d,
$$

and then

$$
T_{p} \mathcal{M}=d \varphi(0)\left[X_{p}\right]=i d\left[X_{p}\right]=X_{p},
$$

or more explicitly

$$
T_{p} \mathcal{M}=\{v \in E:(\nabla J(p), v)=0\} .
$$

Now let $I \in C^{1}(E, \mathbb{R})$ be a functional. We have seen before that if $z \in \mathcal{M}$ is a constrained critical point of $I$ on $\mathcal{M}$,

$$
(\nabla I(z), v)=0, \quad \forall v \in T_{z} \mathcal{M},
$$

and hence by (1.5.4), there is $\lambda \in \mathbb{R}$ that satisfies

$$
\nabla I(z)=\lambda \nabla J(z) .
$$


On the other hand, recall that the constrained gradient is the projection onto $T_{p} \mathcal{M}$ of the free gradient, so that in our actual case

$$
\nabla_{\mathcal{M}} I(p)=\nabla I(p)-(\nabla I(p), w) w=\nabla I(p)-\frac{(\nabla I(p), \nabla J(p))}{\|\nabla J(p)\|^{2}} \nabla J(p),
$$

and thus, if $z$ is a constrained critical point, $\nabla_{\mathcal{M}} I(z)=0$, from which (1.5.5) holds with

$$
\lambda=\frac{(\nabla I(p), \nabla J(p))}{\|\nabla J(p)\|^{2}} .
$$

We close this section with the notion of natural constraint. Given a functional $I \in C^{1}(E, \mathbb{R})$, we say that a manifold $\mathcal{M}$ is a natural constraint for $I$ if there exists a functional $\widetilde{I} \in C^{1}(E, \mathbb{R})$ such that all the critical points of $\widetilde{I}$ constrained on $\mathcal{M}$ are critical points of $I$, i.e.

$$
z \in \mathcal{M}, \nabla_{\mathcal{M}} \widetilde{I}(z)=0 \quad \Leftrightarrow \quad \nabla I(z)=0 .
$$

The Nehari manifold, that it will be relevant in Chapter 2, is an example of a natural constraint.

Definition 1.5.10. Let $I \in C^{1}(E, \mathbb{R})$ be a functional. The Nehari set associated to $I$ is given by

$$
\mathcal{N}:=\left\{u \in E \backslash\{0\}: I^{\prime}(u)[u]=0\right\} .
$$

Proposition 1.5.11. Assume that $I \in C^{2}(E, \mathbb{R})$ and that the Nehari set $\mathcal{N}$ is nonempty. Furthermore, suppose that

$$
I^{\prime \prime}(u)[u, u] \neq 0, \forall u \in \mathcal{N} .
$$

Then, $\mathcal{N}$ is a manifold (called the Nehari manifold), and it is a natural constraint for $I$.

Proof. Let us define the functional $J$ by $J(u):=I^{\prime}(u)[u]$. Then

(i) $J \in C^{1}(E, \mathbb{R})$

(ii) If $u \in \mathcal{N}$, by (1.5.6)

$$
J^{\prime}(u)[u]=I^{\prime \prime}(u)[u, u]+I^{\prime}(u)[u]=I^{\prime \prime}(u)[u, u] \neq 0 .
$$

Hence $J^{\prime}(u) \neq 0$ for $u \in \mathcal{N}$.

These facts let us conclude that $\mathcal{N}$ is a manifold of codimension one. Assume that $z \in \mathcal{N}$ is a constrained critical point of $I$ on $\mathcal{N}$. Therefore for some $\lambda \in \mathbb{R}$ we have

$$
\nabla I(z)=\lambda \nabla J(z)
$$

But then

$$
0=I^{\prime}(z)[z]=(\nabla I(z), z)=\lambda(\nabla J(z), z)=\lambda J^{\prime}(z)[z], \quad \text { with } J^{\prime}(z)[z] \neq 0,
$$

and thus $\lambda=0$. Thence $\nabla I(z)=0$, i.e., $z$ is a critical point of the (free) functional $I$. On the other hand, if $z \neq 0$ is a critical point of $I$ then

$$
\nabla I(z)=0 \quad \Leftrightarrow \quad I^{\prime}(z)=0 .
$$

In particular, $I^{\prime}(z)[z]=0$, so that $z \in \mathcal{N}$. Also it is clear that $\nabla_{\mathcal{N}} I(z)=0$, and the proof is complete.

Example 1.5.12. Let us illustrate that the Nehari manifold is a natural constraint for the reduced functional described in Section 1.4, but with $p$ more restricted: $4<p<6$. There we studied the 
system (1.4.1), namely

$$
\begin{cases}-\Delta u+u+\phi u=|u|^{p-2} u & \text { in } \mathbb{R}^{3} \\ -\Delta \phi=u^{2} & \text { in } \mathbb{R}^{3} .\end{cases}
$$

Briefly, we saw that the solutions of this system are the critical points of the functional $C^{1}$

$$
F(u, \phi)=\frac{1}{2} \int_{\mathbb{R}^{3}}\left[|\nabla u|^{2}+(1+\phi) u^{2}\right] d x-\frac{1}{p} \int_{\mathbb{R}^{3}}|u|^{p} d x-\frac{1}{4} \int_{\mathbb{R}^{3}}|\nabla \phi|^{2} d x,
$$

defined on $H^{1}\left(\mathbb{R}^{3}\right) \times D^{1,2}\left(\mathbb{R}^{3}\right)$. Also that after fixing $u \in H^{1}\left(\mathbb{R}^{3}\right)$, by Proposition 1.4 .5 , to solve the system means to find the critical points of the reduced functional $I(u):=F\left(u, \phi_{u}\right)$, where $\phi_{u} \in D^{1,2}\left(\mathbb{R}^{3}\right)$ denotes the unique solution of

$$
-\Delta \phi=u^{2} \text { in } \mathbb{R}^{3} .
$$

The explicit form of $I$ was determined to be

$$
I(u)=\frac{1}{2} \int_{\mathbb{R}^{3}}\left(|\nabla u|^{2}+u^{2}\right) d x+\frac{1}{4} \int_{\mathbb{R}^{3}} \phi_{u} u^{2} d x-\frac{1}{p} \int_{\mathbb{R}^{3}}|u|^{p} d x .
$$

Now we start our task. It can be shown that $I \in C^{2}\left(H^{1}\left(\mathbb{R}^{3}\right), \mathbb{R}\right)$, and that for every $v, w \in H^{1}\left(\mathbb{R}^{3}\right)$

$$
\begin{aligned}
I^{\prime}(u)[v] & =\int_{\mathbb{R}^{3}}(\nabla u \cdot \nabla v+u v) d x+\int_{\mathbb{R}^{3}} \phi_{u} u v d x-\int_{\mathbb{R}^{3}}|u|^{p-2} u v d x, \\
I^{\prime \prime}(u)[v, w] & =\int_{\mathbb{R}^{3}}(\nabla v \cdot \nabla w+v w) d x+\int_{\mathbb{R}^{3}}\left(\phi_{u} v w+2 \phi_{u, w} u v\right) d x-(p-1) \int_{\mathbb{R}^{3}}|u|^{p-2} v w d x,
\end{aligned}
$$

where, once $u, w \in H^{1}\left(\mathbb{R}^{3}\right)$ are fixed, $\phi_{u, w}$ is the unique solution to

$$
-\Delta \phi=u w \text { in } \mathbb{R}^{3} \text {. }
$$

Indeed if $\phi$ is a solution, for every $\psi \in D^{1,2}\left(\mathbb{R}^{3}\right)$,

$$
\int_{\mathbb{R}^{3}} \nabla \phi \cdot \nabla \psi d x=\int_{\mathbb{R}^{3}} \psi u w d x, \quad T_{u, w}[\psi]:=\int_{\mathbb{R}^{3}} \psi u w d x .
$$

It is clear that $T_{u, w}$ is linear. Also it is continuous because of (1.4.3). Since $T_{u, w} \in D^{\prime}$, by Riesz representation theorem, there is a unique solution $\phi_{u, w} \in D^{1,2}\left(\mathbb{R}^{3}\right)$. In particular, when $u=w$, we have $T_{u}:=T_{u, u}$, the linear continuous operator used in Section 1.4. The Nehari set for $I$ is defined by the condition

$$
I^{\prime}(u)[u]=0 \quad \Leftrightarrow \quad \int_{\mathbb{R}^{3}}\left(|\nabla u|^{2}+u^{2}\right) d x+\int_{\mathbb{R}^{3}} \phi_{u} u^{2} d x=\int_{\mathbb{R}^{3}}|u|^{p} d x .
$$

We remark that

$$
\mathcal{N} \text { is nonempty. }
$$

In fact, considering $u \in H^{1}\left(\mathbb{R}^{3}\right), u \neq 0$, and taking $t>0$, we have

$$
I(t u)=\frac{t^{2}}{2} \int_{\mathbb{R}^{3}}\left(|\nabla u|^{2}+u^{2}\right) d x+\frac{t^{4}}{4} \int_{\mathbb{R}^{3}} \phi_{u} u^{2} d x-\frac{t^{p}}{p} \int_{\mathbb{R}^{3}}|u|^{p} d x .
$$

Hence one can easily see that

- $I(t u)$ has a strict local minimum at $t=0$

- Since $I(t u) \rightarrow-\infty$ when $t \rightarrow \infty$, there is $T>0$ large enough such that $I(T u)<0$. 
Therefore, for some $t_{u}>0, t_{u} u \in \mathcal{N}$. Now for every $u \in \mathcal{N}$, using (1.5.7)

$$
\begin{aligned}
I^{\prime \prime}(u)[u, u] & =\int_{\mathbb{R}^{3}}\left(|\nabla u|^{2}+u^{2}\right) d x+3 \int_{\mathbb{R}^{3}} \phi_{u} u^{2} d x-(p-1) \int_{\mathbb{R}^{3}}|u|^{p} d x \\
& <3\left[\int_{\mathbb{R}^{3}}\left(|\nabla u|^{2}+u^{2}\right) d x+\int_{\mathbb{R}^{3}} \phi_{u} u^{2} d x\right]-(p-1) \int_{\mathbb{R}^{3}}|u|^{p} d x \\
& =3 \int_{\mathbb{R}^{3}}|u|^{p} d x-(p-1) \int_{\mathbb{R}^{3}}|u|^{p} d x \\
& =-(p-4) \int_{\mathbb{R}^{3}}|u|^{p} d x<0,
\end{aligned}
$$

and (1.5.6) is satisfied. In virtue of Proposition 1.5.11, $\mathcal{N}$ is a natural constraint for $I$.

By the way, from (1.5.7) and the continuous Sobolev embedding

$$
\|u\|^{2} \leq|u|_{p}^{p} \leq C\|u\|^{p}
$$

and thus

$$
0<\frac{1}{C} \leq\|u\|^{p-2},
$$

so that $\mathcal{N}$ is bounded away from zero.

\subsection{The Ljusternick-Schnirelmann category}

The Ljusternick-Schnirelmann theory is a key ingredient in order to obtain results of multiplicity of solutions for some elliptic partial differential equations, relying on the topological properties of a suitable set. We give here some basic elements of this theory, that will be used in the main result of the Chapter 2. The exposition is based on Ambrosetti and Malchiodi (2007, Chapter 9).

Definition 1.6.1. Let $\mathcal{M}$ be a topological space; $A \subset \mathcal{M}$ is contractible if the inclusion $A \hookrightarrow \mathcal{M}$ is homotopic to a constant map defined on $A$ with value in $\mathcal{M}$, i.e., there is $H \in C([0,1] \times A, \mathcal{M})$ such that

$$
H(0, u)=u, \quad \text { and } \quad H(1, u)=p, \quad \forall u \in A ; p \text { is a fixed element of } \mathcal{M}
$$

In this section, we assume that $\mathcal{M}$ is a manifold given by

$$
\mathcal{M}=J^{-1}(0), \quad \text { with } J \in C^{1}(E, \mathbb{R}) \text {. Furthermore, } \quad J^{\prime}(u) \neq 0, \forall u \in \mathcal{M},
$$

where $E$ is a Hilbert space.

Definition 1.6.2. Let $\mathcal{M}$ be a topological space. The Ljusternick-Schnirelmann category (or $L S$ category, or simply the category) of $A$ with respect to $\mathcal{M}$ is defined by

$$
\operatorname{cat}_{\mathcal{M}}(A):= \begin{cases}0, & \text { if } A=\emptyset, \\ k, & \text { if } k \text { is the least natural number } l \text { in the property }(C),\end{cases}
$$

where

(C) There are $A_{1}, \ldots, A_{l}$ closed, contractible subsets in $\mathcal{M}$ with $A \subset A_{1} \cup \cdots \cup A_{l}$.

If $A \neq \emptyset$ and $A$ does not satisfy property $(C)$, we set $\operatorname{cat}_{\mathcal{M}}(A)=\infty$.

A last word about notation: we write $\operatorname{cat} \mathcal{M}:=\operatorname{cat}_{\mathcal{M}}(\mathcal{M})$.

Remark 1.6.3. We now list some immediate consequences of the previous definition. 
1. If $A \subset B$ subsets of $\mathcal{M}$, $\operatorname{cat}_{\mathcal{M}}(A) \leq \operatorname{cat}_{\mathcal{M}}(B)$.

Any covering of $B$ by subsets of $\mathcal{M}$ is a covering of $A$ by subsets of $\mathcal{M}$ and the statement follows.

2. $\operatorname{cat}_{\mathcal{M}}(A)=\operatorname{cat}_{\mathcal{M}}(\bar{A})$.

The item easily follows if $A=\emptyset$ or if $\operatorname{cat}_{\mathcal{M}}(A)=\infty$. Assume that $A \neq \emptyset$, $\operatorname{cat}_{\mathcal{M}}(A)=k$.

Therefore, there are $A_{1}, \ldots, A_{k}$ closed, contractible sets in $\mathcal{M}$ with

$$
A \subset A_{1} \cup \cdots \cup A_{k}
$$

Since $A_{1} \cup \cdots \cup A_{k}$ is closed in $\mathcal{M}$ and it contains $A$,

$$
\bar{A} \subset A_{1} \cup \cdots \cup A_{k},
$$

and thus

$$
\operatorname{cat}_{\mathcal{M}}(\bar{A}) \leq k=\operatorname{cat}_{\mathcal{M}}(A) .
$$

On the other hand by the previous item, since $A \subset \bar{A}$, $\operatorname{cat}_{\mathcal{M}}(A) \leq \operatorname{cat}_{\mathcal{M}}(\bar{A})$.

3. $A \subset \mathcal{M} \subset \mathcal{N}$ with $\mathcal{M}$ closed in $\mathcal{N}$ implies $\operatorname{cat}_{\mathcal{M}}(A) \geq \operatorname{cat}_{\mathcal{N}}(A)$.

Definition 1.6.4. Let $\mathcal{M}$ be a topological space; a deformation of $A \subset \mathcal{M}$ in $\mathcal{M}$ is a map $\eta \in$ $C(A, \mathcal{M})$ homotopic to the inclusion $A \hookrightarrow \mathcal{M}$, i.e., there exists $H \in C([0,1] \times A, \mathcal{M})$ such that

$$
H(0, u)=u, \quad H(1, u)=\eta(u), \quad \forall u \in A .
$$

Through this section, the following topological fact will be used in some arguments (see Munkres 2000, Lemma 18.3, pg. 106).

Lemma 1.6.5 (Pasting lemma). Let $X, Y$ be topological spaces. Assume that $X=A \cup B$, where $A, B$ are closed. Let $f: A \rightarrow Y$ and $g: B \rightarrow Y$ be continuous. If $f(x)=g(x)$, for every $x \in A \cap B$, then we can define a continuous function $h: X \rightarrow Y$ using $f$ and $g$, namely by putting $h(x):=f(x)$, for every $x \in A$, and $h(x):=g(x)$, for every $x \in B$.

Lemma 1.6.6. Let $A, B$ subsets of $\mathcal{M}$.

1. $A \subset B \Rightarrow \operatorname{cat}_{\mathcal{M}}(A) \leq \operatorname{cat}_{\mathcal{M}}(B)$

2. $\operatorname{cat}_{\mathcal{M}}(A \cup B) \leq \operatorname{cat}_{\mathcal{M}}(A)+\operatorname{cat}_{\mathcal{M}}(B)$

3. Let $A$ be closed in $\mathcal{M}, \eta$ a deformation of $A$ in $\mathcal{M}$. Then $\operatorname{cat}_{\mathcal{M}}(A) \leq \operatorname{cat}_{\mathcal{M}}(\overline{\eta(A)})$

Proof. 1. The monotonicity of cat was already proved.

2. If $\operatorname{cat}_{\mathcal{M}}(A)=\infty$ or $\operatorname{cat}_{\mathcal{M}}(B)=\infty$, the result is immediate. Let us assume that $\operatorname{cat}_{\mathcal{M}}(A)=k$ and $\operatorname{cat}_{\mathcal{M}}(B)=l$. If $A \subset A_{1} \cup \cdots \cup A_{k}$ and $B \subset B_{1} \cup \cdots \cup B_{l}$, with $A_{i}, B_{j}$ closed, contractible in $\mathcal{M}$, then

$$
A \cup B \subset\left(A_{1} \cup \cdots \cup A_{k}\right) \cup\left(B_{1} \cup \cdots \cup B_{l}\right),
$$

and the item follows.

3. Again if $\operatorname{cat}_{\mathcal{M}}(\eta(A))=\infty$, we do not have anything to prove. Assume that $\operatorname{cat}_{\mathcal{M}}(\eta(A))=k$, so that there are $B_{1}, \ldots, B_{k}$ closed, contractible subsets in $\mathcal{M}$ such that

$$
\eta(A) \subset B_{1} \cup \cdots \cup B_{k} .
$$

Taking $A_{i}:=\eta^{-1}\left(B_{i}\right), A_{i}$ is closed in $A$, and since $A$ is closed in $\mathcal{M}$, it is also closed in $\mathcal{M}$. Furthermore

$$
A \subset A_{1} \cup \cdots \cup A_{k} .
$$


We claim that $A_{i}$ is contractible in $\mathcal{M}$. Indeed, since $\eta$ is a deformation of $A$ in $\mathcal{M}$ and $B_{i}$ contractible in $\mathcal{M}$, we have, respectively, maps $H \in C([0,1] \times A, \mathcal{M})$ with

$$
H(0, u)=u, \quad H(1, u)=\eta(u), \quad \forall u \in A,
$$

and $H_{i} \in C\left([0,1] \times B_{i}, \mathcal{M}\right)$ such that

$$
H_{i}(0, v)=v, \quad H_{i}(1, v)=p_{i}, \quad \forall v \in B_{i}, p_{i} \text { fixed element in } \mathcal{M} .
$$

We define the map $\widetilde{H}_{i}:[0,1] \times A_{i} \rightarrow \mathcal{M}$ by

$$
\widetilde{H}_{i}(t, u):= \begin{cases}H(2 t, u), & \text { for } 0 \leq t \leq 1 / 2 \\ H_{i}(2 t-1, \eta(u)), & \text { for } 1 / 2 \leq t \leq 1,\end{cases}
$$

for every $u \in A_{i}$. As a consequence of the pasting lemma $\widetilde{H}_{i} \in C([0,1] \times \mathcal{M})$ and it satisfies

$$
\widetilde{H}_{i}(0, u)=u, \quad \widetilde{H}_{i}(1, v)=p_{i}, \quad \forall u \in A_{i} .
$$

Hence $A_{i}$ is contractible in $\mathcal{M}$, from which

$$
\operatorname{cat}_{\mathcal{M}}(A) \leq k=\operatorname{cat}_{\mathcal{M}}(\eta(A))=\operatorname{cat}_{\mathcal{M}}(\overline{\eta(A)})
$$

Now we establish a notion that the manifolds that are considered in this thesis will satisfy. This notion leads to a crucial lemma, giving a property of cat when it is calculated on compact sets of the manifold $\mathcal{M}$.

Definition 1.6.7 (Extension property). Let $\mathcal{M}$ be a metric space. $\mathcal{M}$ satisfies the extension property if for every metric space $\mathcal{Y}$, every subset $S$ closed in $\mathcal{Y}$ and every continuous map $f: S \rightarrow$ $\mathcal{M}$, there are $N$ a neighborhood of $S$ in $\mathcal{Y}$ and a map $\widetilde{f} \in C(N, \mathcal{M})$ such that $\left.\widetilde{f}\right|_{S}=f$.

Before the statement of the mentioned crucial lemma, we give a result about metric spaces; it will be used in the proof (see Munkres 2000, Exercise 2, pg. 177).

Proposition 1.6.8. Let $\mathcal{X}$ be a metric space and $A \subset \mathcal{X}$. The $\varepsilon$-neighborhood of $A$ in $\mathcal{X}$ is defined as

$$
U(A, \varepsilon):=\{x \in \mathcal{X}: d(x, A)<\varepsilon\} .
$$

1. $U(A, \varepsilon)$ is the union of the open balls $B_{d}(a, \varepsilon)$, for $a \in A$. Here

$$
B_{d}(a, \varepsilon):=\{y \in \mathcal{X}: d(a, y)<\varepsilon\}
$$

2. If $A$ is compact and $U$ is an open set in $\mathcal{X}$ containing $A$, there is $\varepsilon>0$ such that the $\varepsilon$ neighborhood $U(A, \varepsilon)$ of $A$ is contained in $U$.

Lemma 1.6.9. Let $\mathcal{M}$ be a metric space which satisfies the extension property and let $A \subset \mathcal{M}$ be a compact subset. Then

(1) $\operatorname{cat}_{\mathcal{M}}(A)<\infty$

(2) There exists $U_{A}$ neighborhood of $A$ in $\mathcal{M}$ such that $\operatorname{cat}_{\mathcal{M}}\left(\bar{U}_{A}\right)=\operatorname{cat}_{\mathcal{M}}(A)$.

Proof. Step 1 For $A \subset \mathcal{M}$ compact with cat $\mathcal{M}(A)=1$, there is $U_{A}$ neighborhood of $A$ in $\mathcal{M}$ such that $\operatorname{cat}_{\mathcal{M}}\left(\bar{U}_{A}\right)=1$. 
Since $\operatorname{cat}_{\mathcal{M}}(A)=1$ we have $A \subset B$, with $B$ closed, contractible in $\mathcal{M}$ and also a homotopy $H_{1}:[0,1] \times B \rightarrow \mathcal{M}$ with

$$
H_{1}(0, u)=u, \quad \text { and } \quad H_{1}(1, u)=p, \quad \text { for every } u \in B ; p \text { fixed element in } \mathcal{M} .
$$

In particular $A$ is contractible, considering $H_{2}:=\left.H_{1}\right|_{[0,1] \times A} \in C([0,1] \times A, \mathcal{M})$ such that

$$
H_{2}(0, u)=u, \quad \text { and } \quad H_{2}(1, u)=p, \quad \text { for every } u \in A .
$$

Note that $\mathcal{Y}:=[0,1] \times \mathcal{M}$ is a metric space, since $\mathcal{M}$ is a metric space. Clearly the set

$$
S:=(\{0\} \times \mathcal{M}) \cup([0,1] \times A) \cup(\{1\} \times \mathcal{M})
$$

is closed in $\mathcal{Y}$. By the pasting lemma the map $H: S \rightarrow \mathcal{M}$ given by

$$
H(t, u):= \begin{cases}u, & \text { for } t=0, u \in \mathcal{M} \\ H_{2}(t, u), & \text { for } t \in[0,1], u \in A \\ p, & \text { for } t=1, u \in \mathcal{M}\end{cases}
$$

satisfies $H \in C(S, \mathcal{M})$. As a consequence of the extension property, there is $N$ neighborhood of $S$ in $\mathcal{Y}$ and a map $\widetilde{H} \in C(N, \mathcal{M})$ such that $\left.\widetilde{H}\right|_{S}=H$. Note that $[0,1] \times A$ is compact, and $[0,1] \times A \subset N$ open in $\mathcal{Y}$. Using the Proposition 1.6.8, there is $U_{A}$ neighborhood of $A$ in $\mathcal{M}$ with $[0,1] \times \bar{U}_{A} \subset N$. Furthermore

$$
\widetilde{H}(0, u)=H(0, u)=u, \quad \text { and } \quad \widetilde{H}(1, u)=H(1, u)=p, \quad \text { for every } u \in \bar{U}_{A}
$$

Thus $\bar{U}_{A}$ is contractible in $\mathcal{M}$ and thence $\operatorname{cat}_{\mathcal{M}}\left(\bar{U}_{A}\right)=1$.

Step 2 Since for every $q \in \mathcal{M}$, $\operatorname{cat}_{\mathcal{M}}(\{q\})=1$, as a particular case of Step 1 there exists a neighborhood $U_{q}$ of $q$ in $\mathcal{M}$ with $\bar{U}_{q}$ contractible in $\mathcal{M}$, so that cat $\mathcal{M}_{\mathcal{M}}\left(\bar{U}_{q}\right)=1$.

Step 3 Proofs of items (1) and (2) of the lemma.

Since $A$ is compact, we can find points $q_{1}, \ldots, q_{k} \in \mathcal{M}$ and corresponding neighborhoods $U_{q_{1}}, \ldots, U_{q_{k}}$ with $\bar{U}_{q_{1}}, \ldots, \bar{U}_{q_{k}}$ contractibles in $\mathcal{M}$, such that

$$
A \subset \bar{U}_{q_{1}} \cup \cdots \cup \bar{U}_{q_{k}},
$$

from which

$$
\operatorname{cat}_{\mathcal{M}}(A) \leq \operatorname{cat}_{\mathcal{M}}\left(\bar{U}_{q_{1}} \cup \cdots \cup \bar{U}_{q_{k}}\right) \leq \operatorname{cat}_{\mathcal{M}}\left(\bar{U}_{q_{1}}\right)+\cdots \operatorname{cat}_{\mathcal{M}}\left(\bar{U}_{q_{k}}\right)=k,
$$

and item (1) of lemma follows.

Now assume that cat $\mathcal{M}(A)=k$. Then there exist $A_{1}, \ldots, A_{k}$ closed, contractible sets in $\mathcal{M}$ with $A \subset A_{1} \cup \cdots \cup A_{k}$. Defining $B_{i}:=A_{i} \cap A$, we have $A \subset B_{1} \cup \cdots \cup B_{k}$, with each $B_{i}$ compact. Using Step 1 , for each $i$ there is $U_{i}$ neighborhood of $B_{i}$ in $\mathcal{M}$ with $\bar{U}_{i}$ contractible in $\mathcal{M}$. Defining

$$
U_{A}:=U_{1} \cup \cdots \cup U_{k},
$$

it is clear that $\bar{U}_{A}:=\bar{U}_{1} \cup \cdots \cup \bar{U}_{k}$ and hence

$$
\operatorname{cat}_{\mathcal{M}}\left(\bar{U}_{A}\right) \leq k=\operatorname{cat}_{\mathcal{M}}(A)
$$

On the other hand, since $A \subset \bar{U}_{A}$, by monotonicity $\operatorname{cat}_{\mathcal{M}}(A) \leq \operatorname{cat}_{\mathcal{M}}\left(\bar{U}_{A}\right)$, and item (2) holds true. 
Definition 1.6.10. Let $\mathcal{M}$ be a manifold of the type (1.6.1). We define

$$
\operatorname{cat}_{k}(\mathcal{M}):=\sup \left\{\operatorname{cat}_{\mathcal{M}}(A): A \subset \mathcal{M}, A \text { compact }\right\} .
$$

Note that if $\mathcal{M}$ is compact, $\operatorname{cat}_{k}(\mathcal{M})=\operatorname{cat} \mathcal{M}$, by the monotonicity property of cat. In this last part of abstract results about the $L S$-category, we consider natural numbers with

$$
m \in \begin{cases}\left\{1, \ldots, \operatorname{cat}_{k}(\mathcal{M})\right\}, & \text { if } \operatorname{cat}_{k}(\mathcal{M})<\infty \\ \mathbb{N}, & \text { if } \operatorname{cat}_{k}(\mathcal{M})=\infty\end{cases}
$$

For $m$ satisfying (1.6.2), we define the collection

$$
\mathscr{C}_{m}:=\left\{A \subset \mathcal{M}: A \text { is compact and } \operatorname{cat}_{\mathcal{M}}(A) \geq m\right\}
$$

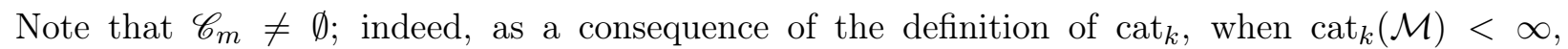
$\operatorname{cat}_{k}(\mathcal{M})=\operatorname{cat}_{\mathcal{M}}(K)$, for some compact subset $K$ in $\mathcal{M}$, and thus $K \in \mathscr{C}_{m}$, for every $m=$ $1, \ldots, \operatorname{cat}_{k}(\mathcal{M})$. When $\operatorname{cat}_{k}(\mathcal{M})=\infty$, for every $m \in \mathbb{N}$, there is $A_{m} \subset \mathcal{M}$ compact such that $m \leq \operatorname{cat}_{\mathcal{M}}\left(A_{m}\right)$, from which $A_{m} \in \mathscr{C}_{m}$.

Now, let us consider $I \in C(\mathcal{M}, \mathbb{R})$. For every $m$ satisfying (1.6.2), we define the minimax levels

$$
c_{m}:=\inf _{A \in \mathscr{C}_{m}} \max _{u \in A} I(u),
$$

which have the following properties:

(i) $c_{1}=\inf _{\mathcal{M}} I$ :

In fact, for every $u \in \mathcal{M}$, $\operatorname{cat}_{\mathcal{M}}(\{u\})=1$, and since $\{u\}$ is compact, we deduce $\{u\} \in \mathscr{C}_{1}$. Thus $c_{1} \leq I(u)$, for every $u \in \mathcal{M}$, and then

$$
c_{1} \leq \inf _{u \in \mathcal{M}} I(u)
$$

follows. On the other hand, $\inf _{u \in \mathcal{M}} I(u) \leq I(v)$, for every $v \in \mathcal{M}$. In particular, for any $v \in A$, with $A \in \mathscr{C}_{m}, \inf _{u \in \mathcal{M}} I(u) \leq \max _{v \in A} I(v)$. Hence

$$
\inf _{u \in \mathcal{M}} I(u) \leq c_{1}
$$

(ii) $c_{1} \leq c_{2} \leq \ldots \leq c_{m-1} \leq c_{m} \leq \ldots$ :

This is immediate from the relation $\mathscr{C}_{m} \subset \mathscr{C}_{m-1}$.

(iii) $c_{m}<\infty$, for every $m$ satisfying (1.6.2):

For any $A \in \mathscr{C}_{m}$,

$$
c_{m} \leq \max _{u \in A} I(u)<\infty,
$$

since $A$ is compact and $I \in C(\mathcal{M}, \mathbb{R})$.

(iv) $\inf _{\mathcal{M}} I>-\infty \Rightarrow c_{m} \in \mathbb{R}$, i.e., $-\infty<c_{1} \leq c_{m}<\infty$ :

Immediate from the hypothesis, because of items (i) - (iii).

Remark 1.6.11. Let $\eta$ be a deformation in $\mathcal{M}$. By the item (3) of Lemma 1.6.6, for any $A \in \mathscr{C}_{m}$,

$$
m \leq \operatorname{cat}_{\mathcal{M}}(A) \leq \operatorname{cat}_{\mathcal{M}}(\eta(A)) .
$$

Furthermore, since $\eta$ is continuous, $\eta(A)$ is compact, and hence $\eta(A) \in \mathscr{C}_{m}$. Summarizing

$$
A \in \mathscr{C}_{m}, \eta \text { deformation in } \mathcal{M} \Rightarrow \eta(A) \in \mathscr{C}_{m}
$$


Now we are going to sketch the proof of the main theorem about the Ljusternick-Schnirelmann theory. For that purpose, we establish some notation, and we state an auxiliary lemma, concerning deformations (see Ambrosetti and Malchiodi 2007, Lemma 7.10, pg. 108 and Lemma 9.12, pg. 151).

- given $a \in \mathbb{R}, \mathcal{M}^{a}:=\{p \in \mathcal{M}: I(p) \leq a\}$, the sublevel associated to $I$ constrained to $\mathcal{M}$ under the level $a$.

- $Z:=\left\{z \in \mathcal{M}: \nabla_{\mathcal{M}} I(z)=0\right\}$, the set of critical points of $I$ on $\mathcal{M}$.

- $\left\{u_{n}\right\} \subset \mathcal{M}$ is a Palais-Smale sequence (PS sequence) on $\mathcal{M}$ for $I$ if $\left\{I\left(u_{n}\right)\right\}$ is bounded and $\nabla_{\mathcal{M}} I\left(u_{n}\right) \rightarrow 0$. It is a $(P S)_{c}$ sequence if $I\left(u_{n}\right) \rightarrow c$ and $\nabla_{\mathcal{M}} I\left(u_{n}\right) \rightarrow 0$. I satifies the $(P S)$ condition on $\mathcal{M}$ if every $P S$ sequence has a convergent subsequence. Similarly, $I$ satifies the $(P S)_{c}$ condition on $\mathcal{M}$ if every $(P S)_{c}$ sequence has a convergent subsequence.

- $Z_{c}:=\{z \in Z: I(z)=c\}$. $c$ is a critical level of $I$ on $\mathcal{M}$ if $Z_{c} \neq \emptyset$. We remark that $Z_{c}$ is compact if $(P S)_{c}$ is satisfied.

Lemma 1.6.12. Let $\mathcal{M}$ be a manifold of the type (1.6.1).

(i) Suppose that $c \in \mathbb{R}$ is not a critical level of $I$ on $\mathcal{M}$ and that $(P S)_{c}$ holds. Then, there exist $\delta>0$ and a deformation $\eta$ in $\mathcal{M}$ such that $\eta\left(\mathcal{M}^{c+\delta}\right) \subset \mathcal{M}^{c-\delta}$.

(ii) Assume that $(P S)_{c}$ holds. For every neighborhood $U$ of $Z_{c}$, there are $\delta>0$ and a deformation $\eta \in \mathcal{M}$ such that

$$
\eta\left(\mathcal{M}^{c+\delta} \backslash U\right) \subset \mathcal{M}^{c-\delta} .
$$

Theorem 1.6.13 (Ljusternick-Schnirelmann theorem). Let $\mathcal{M}$ be a manifold of the type (1.6.1) and $I \in C^{1}(E, \mathbb{R})$ bounded from below on $\mathcal{M}$, satisfying $(P S)$. Then $I$ has at least $\operatorname{cat}_{k}(\mathcal{M})$ critical points on $\mathcal{M}$. More precisely:

(1) any $c_{m}$ is a critical level of $I$ on $\mathcal{M}$.

(2) Suppose that $c:=c_{m}=c_{m+1}=\cdots=c_{m+l}$, for some $l \in \mathbb{N}$. Then $\operatorname{cat}_{\mathcal{M}}\left(Z_{c}\right) \geq l+1$.

Proof. Note that $c_{m} \in \mathbb{R}$, because $I$ is bounded from below on $\mathcal{M}$. We argue by contradiction.

(1) Assume that $Z_{c_{m}}=\emptyset$. Since $I$ is bounded from below on $\mathcal{M}$ and satisfies $(P S)$, by item (i) of Lemma 1.6.12, there are $\delta>0$ and $\eta$ a deformation of $\mathcal{M}$ with

$$
\eta\left(\mathcal{M}^{c_{m}+\delta}\right) \subset \mathcal{M}^{c_{m}-\delta} .
$$

Also, by the definition of $c_{m}$, there exists $A_{m} \in \mathscr{C}_{m}$ such that

$$
I(u) \leq \max _{v \in A_{m}} I(v) \leq c_{m}+\delta, \quad \forall u \in A_{m}
$$

so that $A_{m} \subset \mathcal{M}^{c_{m}+\delta}$. Therefore

$$
\eta\left(A_{m}\right) \subset \mathcal{M}^{c_{m}-\delta} .
$$

But by (1.6.3), $\eta\left(A_{m}\right) \in \mathscr{C}_{m}$, from which, for any $v \in \eta\left(A_{m}\right)$,

$$
c_{m} \leq I(v) \leq \max _{v \in \eta\left(A_{m}\right)} I(v)
$$

in contradiction with (1.6.4).

(2) Let us assume that $\operatorname{cat}_{\mathcal{M}}\left(Z_{c}\right) \leq l$. Since $Z_{c}$ is compact, by Lemma 1.6.9, there is $U$ neighborhood of $Z_{c}$ with

$$
\operatorname{cat}_{\mathcal{M}}(\bar{U})=\operatorname{cat}_{\mathcal{M}}\left(Z_{c}\right) \leq l .
$$


Now by item (ii) of Lemma 1.6.12, there exist $\delta>0$ and $\eta$ a deformation in $\mathcal{M}$ with

$$
\eta\left(\mathcal{M}^{c+\delta} \backslash U\right) \subset \mathcal{M}^{c-\delta}
$$

Since $c=c_{m+l}$, there is $A_{c} \in \mathscr{C}_{m+l}$ such that

$$
I(u) \leq \max _{v \in A_{c}} I(v) \leq c+\delta, \quad \forall u \in A_{c},
$$

and thus

$$
A_{c} \subset \mathcal{M}^{c+\delta}
$$

Now define $B_{c}:=\overline{A_{c} \backslash U}$. Using the monotonicity property of cat,

$$
\bar{A}_{c} \subset \bar{U} \cup B_{c} \Rightarrow \operatorname{cat}_{\mathcal{M}}\left(A_{c}\right)=\operatorname{cat}_{\mathcal{M}}\left(\bar{A}_{c}\right) \leq \operatorname{cat}_{\mathcal{M}}(\bar{U})+\operatorname{cat}_{\mathcal{M}}\left(B_{c}\right),
$$

and thence

$$
\operatorname{cat}_{\mathcal{M}}\left(B_{c}\right) \geq \operatorname{cat}_{\mathcal{M}}\left(A_{c}\right)-\operatorname{cat}_{\mathcal{M}}(\bar{U}) \geq m+l-l=m .
$$

As a consequence, $B_{c} \in \mathscr{C}_{m}$, and by (1.6.3), $\eta\left(B_{c}\right) \in \mathscr{C}_{m}$. In particular, for any $v \in \eta\left(B_{c}\right)$,

$$
c=c_{m} \leq \max _{v \in \eta\left(B_{c}\right)} I(v) .
$$

But on the other hand, it follows from (1.6.5) and (1.6.6) that

$$
\eta\left(B_{c}\right) \subset \mathcal{M}^{c-\delta}
$$

so that $I(v) \leq c-\delta$, a contradiction.

Remark 1.6.14. With respect to the previous theorem and its proof, we want to write explicitly the following facts:

- In Lemma 1.6.12 item (ii) includes item (i), as well as in the Theorem of LjusternickSchnirelmann item (2) includes item (1). Indeed, in the Lemma, if $c$ is not a critical point of $I$ on $\mathcal{M}, U=\emptyset$ is a neighborhood of $Z_{c}=\emptyset$. In the Theorem, item (1) is included in item (2) with $l=0$; when $\operatorname{cat}_{\mathcal{M}}\left(Z_{c}\right) \geq 1$, we have $Z_{c} \neq \emptyset$.

- Item (2) of the Ljusternick-Schnirelmann theorem implies that there are noncountable critical points of I on $\mathcal{M}$ at level $c$. Indeed, since

$$
A \text { discrete set } \Rightarrow \operatorname{cat}_{\mathcal{M}}(A)=1 \text {, }
$$

if item (2) holds true, $Z_{c}$ cannot be countable.

- The proof of the theorem can be done if we have a weaker restriction than $(P S)$; namely, if the minimax levels satisfy

$$
c_{m}<b, \quad \text { for every } m,
$$

then we only need that $(P S)_{c}$ holds for any $c<b$. 


\section{Chapter 2}

\section{Positive semiclassical states for a fractional Schrödinger-Poisson system}

\section{$2.1 \quad$ Introduction}

In the last decades a great attention has been given to the following Schrödinger-Poisson type system

$$
\left\{\begin{array}{l}
-\Delta u+V(x) u+\phi u=|u|^{p-2} u \\
-\Delta \phi=u^{2}
\end{array}\right.
$$

which arises in non relativistic Quantum Mechanics. Such a system is obtained by looking for standing waves solutions in the purely electrostatic case to the Schrödinger-Maxwell system. For a deduction of this system, see e.g. Benci and Fortunato (1998). Here the unknowns are $u$, the modulus of the wave function, and $\phi$ which represents the electrostatic potential. $V$ is a given external potential and $p \geq 2$ a suitable given number.

The system has been studied by many authors, both in bounded and unbounded domains, with different assumptions on the data involved: boundary conditions, potentials, nonlinearities; many different type of solutions have been encountered (minimal energy, sign changing, radial, nonradial...), the behaviour of the solutions (e.g. concentration phenomena) has been studied as well as multiplicity results have been obtained. It is really difficult to give a complete list of references: the reader may see Benci and Fortunato (2014) and the references therein.

However it seems that results relating the number of positive solutions with topological invariants of the "objects" appearing in the problem are few in the literature. We cite the paper Siciliano (2010) where the system is studied in a (smooth and) bounded domain $\Omega \subset \mathbb{R}^{3}$ with $u=\phi=0$ on $\partial \Omega$ and $V$ constant. It is shown, by using variational methods that, whenever $p$ is sufficiently near the critical Sobolev exponent 6 , the number of positive solutions is estimated below by the Ljusternick-Schnirelmann category of the domain $\Omega$.

On the other hand it is known that a particular interest has the semiclassical limit of the Schrödinger-Poisson system (that is when the Plank constant $\hbar$ appearing in the system, see e.g. Benci and Fortunato (1998), tends to zero) especially due to the fact that this limit describes the transition from Quantum to Classical Mechanics. Such a situation is studied e.g. in Ruiz (2005), among many other papers. We cite also Fang and Zhang (2011) which consider the following doubly perturbed system in the whole space $\mathbb{R}^{3}$ :

$$
\left\{\begin{array}{l}
-\varepsilon^{2} \Delta w+V(x) w+\psi w=|w|^{p-2} w \\
-\varepsilon \Delta \psi=w^{2}
\end{array}\right.
$$

Here $V$ is a suitable potential, $4<p<6$, and $\varepsilon$ is a positive parameter proportional to $\hbar$. In this case the authors estimate, whenever $\varepsilon$ tends to zero, the number of positive solutions by the Ljusternick-Schnirelamnn category of the set of minima of the potential $V$, obtaining a result in 
the same spirit of Siciliano (2010).

Recently, especially after the formulation of the Fractional Quantum Mechanics, the derivation of the Fractional Schrödinger equation given by N. Laskin in Laskin (2000a,b, 2002), and the notion of fractional harmonic extension of a function studied in the pioneering paper Caffarelli and Silvestre (2007), equations involving fractional operators are receiving a great attention. Indeed pseudodifferential operators appear in many problems in Physics and Chemistry, see e.g. Metzler and Klafter (2000, 2004); but also in obstacle problems Milakis and Silvestre (2008); Silvestre (2007), optimization and finance Cont and Tankov (2004), conformal geometry and minimal surfaces Caffarelli and Valdinoci (2011); Caffarelli et al. (2010); Chang and del Mar González (2011), etc.

Motivated by the previous discussion, we investigate in this chapter the existence of positive solutions for the following doubly singularly perturbed fractional Schrödinger-Poisson system in $\mathbb{R}^{N}$ :

$$
\left\{\begin{array}{l}
\varepsilon^{2 s}(-\Delta)^{s} w+V(x) w+\psi w=f(w) \\
\varepsilon^{\theta}(-\Delta)^{\alpha / 2} \psi=\gamma_{\alpha} w^{2}
\end{array}\right.
$$

where $\gamma_{\alpha}:=\frac{\pi^{N / 2} 2^{\alpha} \Gamma(\alpha / 2)}{\Gamma(N / 2-\alpha / 2)}$ is a constant ( $\Gamma$ is the Euler function). By a positive solution of $\left(P_{\varepsilon}\right)$ we mean a pair $(w, \psi)$ where $w$ is positive. To the best of our knowledge, there are only few recent papers dealing with a system like $\left(P_{\varepsilon}\right)$ : in Zhang (2015) the author deals with $\varepsilon=1$ proving under suitable assumptions on $f$ the existence of infinitely many (but possibly sign changing) solutions by means of the Fountain Theorem. A similar system is studied in Wei (2015) and the existence of infinitely many (again, possibly sign changing) solutions is obtained by means of the Symmetric Mountain Pass Theorem.

In this chapter we assume that

(H1) $s \in(0,1), \theta \in(0, \alpha), \max \{\alpha, 2 s\}<N<\min \{4 s, 2 s+\alpha\}$.

These technical conditions lead to the following allowed values for the parameters $s, \alpha$ and $N$ in our study:

\begin{tabular}{|l|l|c|}
\hline$N=1$ & $0<\alpha<\frac{1}{2}$ & $\frac{1-\alpha}{2}<s<\frac{1}{2}$ \\
\cline { 2 - 3 } & $\frac{1}{2} \leq \alpha<1$ & $\frac{1}{4}<s<\frac{1}{2}$ \\
\hline \multirow{2}{*}{$N=2$} & $0<\alpha<1$ & $\frac{2-\alpha}{2}<s<1$ \\
\cline { 2 - 3 } & $1 \leq \alpha<2$ & $\frac{1}{2}<s<1$ \\
\hline \multirow{2}{*}{$N=3$} & $1<\alpha<\frac{3}{2}$ & $\frac{3-\alpha}{2}<s<1$ \\
\cline { 2 - 3 } & $\frac{3}{2} \leq \alpha<3$ & $\frac{3}{4}<s<1$ \\
\hline
\end{tabular}

Moreover the potential $V$ and the nonlinearity $f$ satisfy the assumptions listed below:

(V1) $V: \mathbb{R}^{N} \rightarrow \mathbb{R}$ is a continuous function and

$$
0<\min _{\mathbb{R}^{N}} V:=V_{0}<V_{\infty}:=\liminf _{|x| \rightarrow+\infty} V \in\left(V_{0},+\infty\right]
$$

with $M:=\left\{x \in \mathbb{R}^{N}: V(x)=V_{0}\right\}$ smooth and bounded;

(f1) $f: \mathbb{R} \rightarrow \mathbb{R}$ is a function of class $C^{1}$ and $f(t)=0$ for $t \leq 0$;

(f2) $\lim _{t \rightarrow 0} f(t) / t=0$; 
(f3) there is $q_{0} \in\left(3,2_{s}^{*}-1\right)$ such that $\lim _{t \rightarrow \infty} f(t) / t^{q_{0}}=0$, where $2_{s}^{*}:=2 N /(N-2 s)$;

(f4) there is $K>4$ such that $0<K F(t):=K \int_{0}^{t} f(\tau) d \tau \leq t f(t)$ for all $t>0$;

(f5) for every $t>0, \frac{d}{d t}\left(\frac{f(t)}{t^{3}}\right)>0$.

The assumptions on the nonlinearity $f$ are quite standard in order to work with variational methods, use the Nehari manifold and the Palais-Smale condition. The assumption (V1) will be fundamental in order to estimate the number of positive solutions and also to recover some compactness.

We recall, once for all, that a $C^{1}$ functional $\mathcal{J}$, defined on a smooth manifold $\mathfrak{M}$, is said to satisfy the Palais-Smale condition at level $c \in \mathbb{R}\left((P S)_{c}\right.$ for brevity) if every sequence $\left\{u_{n}\right\} \subset \mathfrak{M}$ such that

$$
\mathcal{J}\left(u_{n}\right) \rightarrow c \quad \text { and } \quad \mathcal{J}^{\prime}\left(u_{n}\right) \rightarrow 0
$$

has a convergent subsequence. A sequence $\left\{u_{n}\right\}$ satisfying (2.1.1) is also named a $(P S)_{c}$ sequence.

Our result is the following

Theorem 2.1.1. Under the above assumptions (H1), (V1), (f1)-(f5), there exists an $\varepsilon^{*}>0$ such that for every $\varepsilon \in\left(0, \varepsilon^{*}\right]$ problem $\left(P_{\varepsilon}\right)$ possesses at least cat $M$ positive solutions.

Moreover if cat $M>1$, then (for suitably small $\varepsilon$ ) there exist at least cat $M+1$ positive solutions.

Hereafter, given a topological pair $(X, Y)$, cat $_{X}(Y)$ is the Ljusternick-Schnirelmann category of $Y$ in $X$, and, if $X=Y$ this is just denoted with cat $X$.

The proof of Theorem 2.1.1 is carried out by adapting some ideas of Benci, Cerami and Passaseo Benci and Cerami (1991); Benci et al. (1991) and using the Ljusternick-Schnirelmann Theory. We mention that these ideas and techniques have been extensively used to attack also other type of problems, and indeed similar results are obtained for other equations and operators, like the Schrödinger operator Cingolani and Lazzo (1997, 2000), the $p$-laplacian Alves and Figueiredo (2006); Alves and Soares (2010), the biharmonic operator Alves and Figueiredo (2013), $p \& q-$ laplacian, fractional laplacian (Figueiredo and Siciliano, 2016; Figueiredo et al., 2015), magnetic laplacian (Alves and Figueiredo, 2014a,b) or quasilinear operators Alves and Figueiredo (2015); Alves et al. (2009, 2012).

The plan of the chapter is the following. In Section 2.2 we recall some basic facts, we present some preliminaries and the variational setting for the problem. Section 2.3 is devoted to prove some compactness properties; as a byproduct we prove the existence of a ground state solution for our problem, that is a solution having minimal energy. In Section 2.4 we introduce the barycenter map, we show some of its properties and prove, by means of the Ljusternick-Schnirelmann Theory, Theorem 2.1.1.

Notations. In the chapter we will denote with $|\cdot|_{p}$ the usual $L^{p}$ norm in $\mathbb{R}^{N}$; we denote with $B_{r}(x)$ the closed ball in $\mathbb{R}^{N}$ centered in $x$ with radius $r>0$, with $B_{r}^{c}(x)$ its complementary; if $x=0$ we simply write $B_{r}$; moreover the letters $C, C_{1}, C_{2}, \ldots$ will denote generic positive constants (whose value may change from line to line). Other notations will be introduced whenever we need.

\subsection{Preliminaries}

\subsubsection{Some well known facts}

Before to introduce the variational setting of our problem, we recall some basic facts concerning the fractional Sobolev spaces and their embeddings. 
Given $\beta \in(0,1)$, the fractional Laplacian $(-\Delta)^{\beta}$ is the pseudodifferential operator which can be defined via the Fourier transform

$$
\mathcal{F}\left((-\Delta)^{\beta} u\right)=|\cdot|^{2 \beta} \mathcal{F} u,
$$

or, if $u$ has sufficient regularity, by

$$
(-\Delta)^{\beta} u(z)=-\frac{C_{N, \beta}}{2} \int_{\mathbb{R}^{N}} \frac{u(z+y)+u(z-y)-2 u(z)}{|y|^{N+2 \beta}} d y, \quad z \in \mathbb{R}^{N},
$$

where $C_{N, \beta}$ is a suitable normalization constant.

For $s \in(0,1)$ let

$$
H^{s}\left(\mathbb{R}^{N}\right)=\left\{u \in L^{2}\left(\mathbb{R}^{N}\right):(-\Delta)^{s / 2} u \in L^{2}\left(\mathbb{R}^{N}\right)\right\}
$$

be the Hilbert space with scalar product and (squared) norm given by

$$
(u, v)=\int_{\mathbb{R}^{N}}(-\Delta)^{s / 2} u(-\Delta)^{s / 2} v+\int_{\mathbb{R}^{N}} u v, \quad\|u\|^{2}=\left|(-\Delta)^{s / 2} u\right|_{2}^{2}+|u|_{2}^{2} .
$$

It is known that $H^{s}\left(\mathbb{R}^{N}\right) \hookrightarrow L^{p}\left(\mathbb{R}^{N}\right), p \in\left[2,2_{s}^{*}\right]$ with $2_{s}^{*}:=2 N /(N-2 s)$. Moreover the embedding of $H^{s}(\Omega)$ is compact if $\Omega \subset \mathbb{R}^{N}$ is bounded and $p \neq 2_{s}^{*}$.

We will consider also the homogeneous Sobolev spaces $\dot{H}^{\alpha / 2}\left(\mathbb{R}^{N}\right)$ defined as the completion of $C_{c}^{\infty}\left(\mathbb{R}^{N}\right)$ with respect to the norm $\left|(-\Delta)^{\alpha / 4} u\right|_{2}$. This is a Hilbert space with scalar product and (squared) norm

$$
(u, v)_{\dot{H}^{\alpha / 2}}=\int_{\mathbb{R}^{N}}(-\Delta)^{\alpha / 4} u(-\Delta)^{\alpha / 4} v, \quad\|u\|_{\dot{H}^{\alpha / 2}}^{2}=\left|(-\Delta)^{\alpha / 4} u\right|_{2}^{2} .
$$

It is well known that $\dot{H}^{\alpha / 2}\left(\mathbb{R}^{N}\right) \hookrightarrow L^{2_{\alpha / 2}^{*}}\left(\mathbb{R}^{N}\right), 2_{\alpha / 2}^{*}=2 N /(N-\alpha)$. For more general facts about the fractional Laplacian we refer the reader to the beautiful paper Nezza et al. (2012).

We recall here another fact that will be frequently used:

$$
\forall \xi>0 \exists M_{\xi}>0: \quad \int_{\mathbb{R}^{N}} f(u) u \leq \xi \int_{\mathbb{R}^{N}} u^{2}+M_{\xi} \int_{\mathbb{R}^{N}}|u|^{q_{0}+1}, \quad \forall u \in H^{s}\left(\mathbb{R}^{N}\right) .
$$

This simply follows by (f1) - (f3).

\subsubsection{The variational setting}

It is easily seen that, just performing the change of variables $u(x):=w(\varepsilon x), \phi(x):=\psi(\varepsilon x)$, problem $\left(P_{\varepsilon}\right)$ can be rewritten as

$$
\left\{\begin{array}{l}
(-\Delta)^{s} u+V(\varepsilon x) u+\phi(x) u=f(u) \\
(-\Delta)^{\alpha / 2} \phi=\varepsilon^{\alpha-\theta} \gamma_{\alpha} u^{2},
\end{array}\right.
$$

to which we will refer from now on.

A usual "reduction" argument can be used to deal with a single equation involving just $u$. Indeed for every $u \in H^{s}\left(\mathbb{R}^{N}\right)$ the second equation in $\left(P_{\varepsilon}^{*}\right)$ is uniquely solved. Actually, for future reference, we will prove a slightly more general fact.

Let us fix two functions $u, w \in H^{s}\left(\mathbb{R}^{N}\right)$ and consider the problem

$$
\left\{\begin{array}{l}
(-\Delta)^{\alpha / 2} \phi=\varepsilon^{\alpha-\theta} \gamma_{\alpha} u w \\
\phi \in \dot{H}^{\alpha / 2}\left(\mathbb{R}^{N}\right)
\end{array}\right.
$$


whose weak solution is a function $\tilde{\phi} \in \dot{H}^{\alpha / 2}\left(\mathbb{R}^{N}\right)$ such that

$$
\forall v \in \dot{H}^{\alpha / 2}\left(\mathbb{R}^{N}\right): \quad \int_{\mathbb{R}^{N}}(-\Delta)^{\alpha / 4} \tilde{\phi}(-\Delta)^{\alpha / 4} v=\varepsilon^{\alpha-\theta} \gamma_{\alpha} \int_{\mathbb{R}^{N}} u w v .
$$

For every $v \in \dot{H}^{\alpha / 2}\left(\mathbb{R}^{N}\right)$, by the Hölder inequality and the continuous embeddings, we have

$$
\left|\int_{\mathbb{R}^{N}} u w v\right| \leq|u|_{\frac{4 N}{N+\alpha}}|w|_{\frac{4 N}{N+\alpha}}|v|_{2_{\alpha / 2}^{*}} \leq C\|u\|\|w\|\|v\|_{\dot{H}^{\alpha / 2}}
$$

deducing that the map

$$
T_{u, w}: v \in \dot{H}^{\alpha / 2}\left(\mathbb{R}^{N}\right) \longmapsto \int_{\mathbb{R}^{N}} u w v \in \mathbb{R}
$$

is linear and continuous: then there exists a unique solution $\phi_{\varepsilon, u, w} \in \dot{H}^{\alpha / 2}\left(\mathbb{R}^{N}\right)$ to $\left(Q_{\varepsilon}\right)$. Moreover this solution has the representation by means of the Riesz kernel $\mathcal{K}_{\alpha}(x)=\gamma_{\alpha}^{-1}|x|^{\alpha-N}$, hence

$$
\phi_{\varepsilon, u, w}=\varepsilon^{\alpha-\theta} \frac{1}{|\cdot|^{N-\alpha}} \star(u w) .
$$

Furthermore

$$
\left\|\phi_{\varepsilon, u, w}\right\|_{\dot{H}^{\alpha / 2}}=\varepsilon^{\alpha-\theta}\left\|T_{u, w}\right\|_{\mathcal{L}\left(\dot{H}^{\alpha / 2} ; \mathbb{R}\right)} \leq \varepsilon^{\alpha-\theta} C\|u\|\|w\|
$$

and then, for $\zeta, \eta \in H^{s}\left(\mathbb{R}^{N}\right)$

$$
\int_{\mathbb{R}^{N}} \phi_{\varepsilon, u, w} \zeta \eta \leq\left|\phi_{\varepsilon, u, w}\right|_{2_{\alpha / 2}^{*}}|\zeta|_{\frac{4 N}{N+\alpha}}|\eta|_{\frac{4 N}{N+\alpha}} \leq \varepsilon^{\alpha-\theta} C_{\mathrm{e}}\|u\|\|w\|\|\zeta\|\|\eta\|
$$

where $C_{\mathrm{e}}$ is a suitable embedding constant. Although its value is not important, we will refer to this constant later on.

A particular case of the previous situation is when $u=w$. In this case we simplify the notation and write

- $T_{u}(v):=T_{u, u}(v)=\int_{\mathbb{R}^{N}} u^{2} v$, and

- $\phi_{\varepsilon, u}$ for the unique solution of the second equation in $\left(P_{\varepsilon}^{*}\right)$ for fixed $u \in H^{s}\left(\mathbb{R}^{N}\right)$. Then

$$
\left\|\phi_{\varepsilon, u}\right\|_{\dot{H}^{\alpha / 2}} \leq \varepsilon^{\alpha-\theta} C\|u\|^{2}
$$

and the map

$$
u \in H^{s}\left(\mathbb{R}^{N}\right) \longmapsto \phi_{\varepsilon, u} \in \dot{H}^{\alpha / 2}\left(\mathbb{R}^{N}\right)
$$

is bounded.

Observe also that

$$
u_{n}^{2} \longrightarrow u^{2} \text { in } L^{\frac{2 N}{N+\alpha}}\left(\mathbb{R}^{N}\right) \Longrightarrow T_{u_{n}} \longrightarrow T_{u} \text { as operators } \Longrightarrow \phi_{\varepsilon, u_{n}} \longrightarrow \phi_{\varepsilon, u} \text { in } \dot{H}^{\alpha / 2}\left(\mathbb{R}^{N}\right)
$$

For convenience let us define the map (well defined by (2.2.3))

$$
A: u \in H^{s}\left(\mathbb{R}^{N}\right) \longmapsto \int_{\mathbb{R}^{N}} \phi_{\varepsilon, u} u^{2} \in \mathbb{R} .
$$

Then

$$
|A(u)| \leq \varepsilon^{\alpha-\theta} C_{\mathrm{e}}\|u\|^{4}
$$

(where $C_{\mathrm{e}}$ is the same constant in (2.2.3)). Some relevant properties of $\phi_{\varepsilon, u}$ and $A$ are listed below. Although these properties are known to be true, we are not able to find them explicitly in the literature; so we prefer to give a proof here. 
Lemma 2.2.1. The following propositions hold.

(i) For every $u \in H^{s}\left(\mathbb{R}^{N}\right): \phi_{\varepsilon, u} \geq 0$;

(ii) for every $u \in H^{s}\left(\mathbb{R}^{N}\right), t \in \mathbb{R}: \phi_{\varepsilon, t u}=t^{2} \phi_{\varepsilon, u}$;

(iii) if $u_{n} \rightarrow u$ in $H^{s}\left(\mathbb{R}^{N}\right)$ then $\phi_{\varepsilon, u_{n}} \rightarrow \phi_{\varepsilon, u}$ in $\dot{H}^{\alpha / 2}\left(\mathbb{R}^{N}\right)$;

(iv) $A$ is of class $C^{2}$ and for every $u, v, w \in H^{s}\left(\mathbb{R}^{N}\right)$

$$
A^{\prime}(u)[v]=4 \int_{\mathbb{R}^{N}} \phi_{\varepsilon, u} u v, \quad A^{\prime \prime}(u)[v, w]=4 \int_{\mathbb{R}^{N}} \phi_{\varepsilon, u} v w+8 \int_{\mathbb{R}^{N}} \phi_{\varepsilon, u, w} u v,
$$

$(v)$ if $u_{n} \rightarrow u$ in $L^{r}\left(\mathbb{R}^{N}\right)$, with $2 \leq r<2_{s}^{*}$, then $A\left(u_{n}\right) \rightarrow A(u)$;

$(v i)$ if $u_{n} \rightarrow u$ in $H^{s}\left(\mathbb{R}^{N}\right)$ then $A\left(u_{n}-u\right)=A\left(u_{n}\right)-A(u)+o_{n}(1)$.

Proof. Items $(i)$ and $(i i)$ follow directly by the definition of $\phi_{\varepsilon, u}$.

To prove $\left(\right.$ iii), let $v \in C_{c}^{\infty}\left(\mathbb{R}^{N}\right)$; we have

$$
\begin{aligned}
\int_{\mathbb{R}^{N}}(-\Delta)^{\alpha / 4}\left(\phi_{\varepsilon, u_{n}}-\phi_{\varepsilon, u}\right)(-\Delta)^{\alpha / 4} v & =\int_{\mathbb{R}^{N}}\left(u_{n}^{2}-u^{2}\right) v \\
& \leq|v|_{\infty}\left(\int_{\operatorname{supp} v}\left(u_{n}-u\right)^{2}\right)^{1 / 2}\left(\int_{\operatorname{supp} v}\left(u_{n}+u\right)^{2}\right)^{1 / 2} \\
& \rightarrow 0 .
\end{aligned}
$$

The conclusion then follows by density.

The proof of $(i v)$ is straightforward: we refer the reader to Fang and Zhang (2011).

To show $(v)$, recall that $2<\frac{4 N}{N+\alpha}<2_{s}^{*}$. Since by assumption $\left|u_{n}^{2}\right|_{\frac{2 N}{N+\alpha}} \rightarrow\left|u^{2}\right|_{\frac{2 N}{N+\alpha}}$ and $u_{n}^{2} \rightarrow u^{2}$ a.e. in $\mathbb{R}^{N}$, using the Brezis-Lieb Lemma, $u_{n}^{2} \rightarrow u^{2}$ in $L^{\frac{2 N}{N+\alpha}}\left(\mathbb{R}^{N}\right)$. But then using (2.2.4) we get $\phi_{\varepsilon, u_{n}} \rightarrow \phi_{\varepsilon, u}$ in $L^{2_{\alpha / 2}^{*}}\left(\mathbb{R}^{N}\right)$. Consequently

$$
\begin{aligned}
\left|A\left(u_{n}\right)-A(u)\right| & \leq \int_{\mathbb{R}^{N}}\left|\phi_{\varepsilon, u_{n}} u_{n}^{2}-\phi_{\varepsilon, u} u^{2}\right| \leq \int_{\mathbb{R}^{N}}\left|\left(\phi_{\varepsilon, u_{n}}-\phi_{\varepsilon, u}\right) u_{n}^{2}\right|+\int_{\mathbb{R}^{N}}\left|\phi_{\varepsilon, u}\left(u_{n}^{2}-u^{2}\right)\right| \\
& \leq\left|\phi_{\varepsilon, u_{n}}-\phi_{\varepsilon, u}\right|_{2_{\alpha / 2}^{*}}\left|u_{n}^{2}\right|_{\frac{2 N}{N+\alpha}}+\left|\phi_{\varepsilon, u}\right|_{2_{\alpha / 2}^{*}}\left|u_{n}^{2}-u^{2}\right|_{\frac{2 N}{N+\alpha}}
\end{aligned}
$$

from which we conclude.

To prove $(v i)$, for the sake of simplicity we drop the factor $\varepsilon^{\alpha-\theta}$ in the expression of $\phi_{\varepsilon, u, w}$. Defining

$$
\begin{gathered}
\sigma:=\int_{\mathbb{R}^{N}} \int_{\mathbb{R}^{N}} \frac{u^{2}(y) u^{2}(x)}{|x-y|^{N-\alpha}} d y d x \\
\sigma_{n}^{1}:=\int_{\mathbb{R}^{N}} \int_{\mathbb{R}^{N}} \frac{u_{n}^{2}(y) u^{2}(x)}{|x-y|^{N-\alpha}} d y d x, \quad \sigma_{n}^{2}:=\int_{\mathbb{R}^{N}} \int_{\mathbb{R}^{N}} \frac{u_{n}(y) u(y) u_{n}(x) u(x)}{|x-y|^{N-\alpha}} d y d x \\
\sigma_{n}^{3}:=\int_{\mathbb{R}^{N}} \int_{\mathbb{R}^{N}} \frac{u_{n}^{2}(y) u_{n}(x) u(x)}{|x-y|^{N-\alpha}} d y d x, \quad \sigma_{n}^{4}:=\int_{\mathbb{R}^{N}} \int_{\mathbb{R}^{N}} \frac{u_{n}(y) u(y) u^{2}(x)}{|x-y|^{N-\alpha}} d y d x,
\end{gathered}
$$

it is easy to check that

$$
A\left(u_{n}-u\right)-A\left(u_{n}\right)+A(u)=2 \sigma+2 \sigma_{n}^{1}+4 \sigma_{n}^{2}-4 \sigma_{n}^{3}-4 \sigma_{n}^{4} .
$$


Now we claim that, whenever $u_{n} \rightarrow u$ in $H^{s}\left(\mathbb{R}^{N}\right)$,

$$
\lim _{n \rightarrow \infty} \sigma_{n}^{i}=\sigma, \quad i=1,2,3,4
$$

which readily gives the conclusion.

We prove here only the cases $i=1,2$ since the proof of the other cases is very similar. Recall that

$$
\phi_{\varepsilon, u}(x)=\int_{\mathbb{R}^{N}} \frac{u^{2}(y)}{|x-y|^{N-\alpha}} d y, \quad \phi_{\varepsilon, u_{n}}(x)=\int_{\mathbb{R}^{N}} \frac{u_{n}^{2}(y)}{|x-y|^{N-\alpha}} d y .
$$

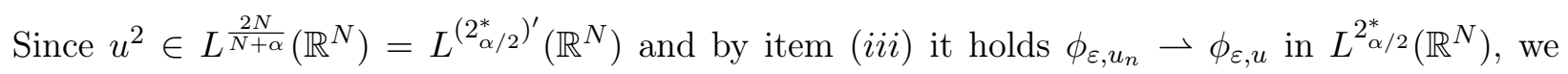
conclude that

$$
\sigma_{n}^{1}=\int_{\mathbb{R}^{N}} \phi_{\varepsilon, u_{n}} u^{2} \rightarrow \int_{\mathbb{R}^{N}} \phi_{\varepsilon, u} u^{2}=\sigma
$$

and the claim is true for $i=1$.

For $i=2$ recall that

$$
\phi_{\varepsilon, u_{n}, u}(x)=\int_{\mathbb{R}^{N}} \frac{u_{n}(y) u(y)}{|x-y|^{N-\alpha}} d y .
$$

First we show that $\phi_{\varepsilon, u_{n}, u} \rightarrow \phi_{\varepsilon, u}$ a.e. in $\mathbb{R}^{N}$. Given $\xi>0$ and choosing $R>1 / \xi, \frac{N}{2 s}<p<\frac{N}{N-\alpha}$ and $\frac{N}{N-\alpha}<q$ (so that $2 p^{\prime}, 2 q^{\prime} \in\left(2,2_{s}^{*}\right)$ ), we have, for large $n$ :

$$
\begin{aligned}
& \left|\phi_{\varepsilon, u_{n}, u}(x)-\phi_{\varepsilon, u}(x)\right| \leq\left|u_{n}-u\right|_{L^{2 p^{\prime}}\left(B_{R}(x)\right)}|u|_{L^{2 p^{\prime}}\left(B_{R}(x)\right)}\left(\int_{|y-x|<R} \frac{d y}{|x-y|^{p(N-\alpha)}}\right)^{1 / p} \\
& +\left|u_{n}-u\right|_{L^{2 q^{\prime}\left(B_{R}^{c}(x)\right)}}|u|_{L^{2 q^{\prime}\left(B_{R}^{c}(x)\right)}}\left(\int_{|y-x| \geq R} \frac{d y}{|x-y|^{q(N-\alpha)}}\right)^{1 / q} \\
& \leq C_{1} \xi+C_{2} \xi^{N-\alpha-N / q}
\end{aligned}
$$

concluding the pointwise convergence. Moreover by the Sobolev embedding and using (2.2.2),

$$
\left|\phi_{\varepsilon, u_{n}, u} u_{n}\right|_{2} \leq\left|\phi_{\varepsilon, u_{n}, u}\right|_{2_{\alpha / 2}^{*}}\left|u_{n}\right|_{2 N / \alpha} \leq C_{1}\left\|u_{n}\right\|^{2}\|u\| \leq C_{2}
$$

and therefore, up to subsequence, $\phi_{\varepsilon, u_{n}, u} u_{n} \rightarrow \phi_{\varepsilon, u} u$ in $L^{2}\left(\mathbb{R}^{N}\right)$, by Kavian (1993, Lemma 4.8). Since $u \in L^{2}\left(\mathbb{R}^{N}\right)$

$$
\sigma_{n}^{2}=\int_{\mathbb{R}^{N}} \phi_{\varepsilon, u_{n}, u} u_{n} u \rightarrow \int_{\mathbb{R}^{N}} \phi_{\varepsilon, u} u^{2}=\sigma
$$

and the claim is proved for $i=2$.

We introduce now the variational setting for our problem. Let us define the Hilbert space

$$
W_{\varepsilon}:=\left\{u \in H^{s}\left(\mathbb{R}^{N}\right): \int_{\mathbb{R}^{N}} V(\varepsilon x) u^{2}<\infty\right\}
$$

endowed with scalar product and (squared) norm given by

$$
(u, v)_{\varepsilon}:=\int_{\mathbb{R}^{N}}(-\Delta)^{s / 2} u(-\Delta)^{s / 2} v+\int_{\mathbb{R}^{N}} V(\varepsilon x) u v
$$

and

$$
\|u\|_{\varepsilon}^{2}:=\int_{\mathbb{R}^{N}}\left|(-\Delta)^{s / 2} u\right|^{2}+\int_{\mathbb{R}^{N}} V(\varepsilon x) u^{2}
$$


Then it is standard to see that the critical points of the $C^{2}$ functional (see Lemma 2.2.1 (iv))

$$
I_{\varepsilon}(u):=\frac{1}{2} \int_{\mathbb{R}^{N}}\left|(-\Delta)^{s / 2} u\right|^{2}+\frac{1}{2} \int_{\mathbb{R}^{N}} V(\varepsilon x) u^{2}+\frac{1}{4} \int_{\mathbb{R}^{N}} \phi_{\varepsilon, u} u^{2}-\int_{\mathbb{R}^{N}} F(u),
$$

on $W_{\varepsilon}$ are weak solutions of problem $\left(P_{\varepsilon}^{*}\right)$.

By defining

$$
\mathcal{N}_{\varepsilon}:=\left\{u \in W_{\varepsilon} \backslash\{0\}: J_{\varepsilon}(u)=0\right\}
$$

where

$$
J_{\varepsilon}(u):=I_{\varepsilon}^{\prime}(u)[u]=\|u\|_{\varepsilon}^{2}+\int_{\mathbb{R}^{N}} \phi_{\varepsilon, u} u^{2}-\int_{\mathbb{R}^{N}} f(u) u,
$$

we have, by standard arguments:

Lemma 2.2.2. For every $u \in \mathcal{N}_{\varepsilon}, J_{\varepsilon}^{\prime}(u)[u]<0$ and there are positive constants $h_{\varepsilon}, k_{\varepsilon}$ such that $\|u\|_{\varepsilon} \geq h_{\varepsilon}, I_{\varepsilon}(u) \geq k_{\varepsilon}$. Furthermore, $\mathcal{N}_{\varepsilon}$ is diffeomorphic to the set

$$
\mathcal{S}_{\varepsilon}:=\left\{u \in W_{\varepsilon}:\|u\|_{\varepsilon}=1\right\} \backslash\left\{u \in W_{\varepsilon}: u \leq 0 \text { a.e. }\right\} \text {. }
$$

$\mathcal{N}_{\varepsilon}$ is the Nehari manifold associated to $I_{\varepsilon}$. By the assumptions on $f$, the functional $I_{\varepsilon}$ has the Mountain Pass geometry. This is standard but we give the easy proof for completeness.

$(\mathrm{MP} 1) I_{\varepsilon}(0)=0$

(MP2) since, for every $\xi>0$ there exists $M_{\xi}>0$ such that $F(u) \leq \xi u^{2}+M_{\xi}|u|^{q_{0}+1}$, we have

$$
\begin{aligned}
I_{\varepsilon}(u) & \geq \frac{1}{2}\|u\|_{\varepsilon}^{2}-\int_{\mathbb{R}^{N}} F(u) \\
& \geq \frac{1}{2}\|u\|_{\varepsilon}^{2}-\xi C_{1}\|u\|_{\varepsilon}^{2}-M_{\xi} C_{2}\|u\|_{\varepsilon}^{q_{0}+1}
\end{aligned}
$$

and we conclude $I_{\varepsilon}$ has a strict local minimum at $u=0$;

(MP3) finally, since (f4) implies $F(t) \geq C t^{K}$ for large $t>0$, with $C>0, K>4$ (and less then $q_{0}+1$ ), fixed $v \in C_{c}^{\infty}\left(\mathbb{R}^{N}\right), v>0$ we have

$$
\begin{aligned}
I_{\varepsilon}(t v) & =\frac{t^{2}}{2}\|v\|_{\varepsilon}^{2}+\frac{t^{4}}{4} \int_{\mathbb{R}^{N}} \phi_{\varepsilon, v} v^{2}-\int_{\mathbb{R}^{N}} F(t v) \\
& \leq \frac{t^{2}}{2}\|v\|_{\varepsilon}^{2}+\frac{t^{4}}{4} \int_{\mathbb{R}^{N}} \phi_{\varepsilon, v} v^{2}-C t^{K} \int_{\mathbb{R}^{N}} v^{K}
\end{aligned}
$$

concluding that the functional is negative for suitable large $t$.

Then denoting with

$$
c_{\varepsilon}:=\inf _{\gamma \in \mathcal{H}} \sup _{\varepsilon \in[0,1]} I_{\varepsilon}(\gamma(t)), \quad \mathcal{H}_{\varepsilon}=\left\{\gamma \in C\left([0,1], W_{\varepsilon}\right): \gamma(0)=0, I_{\varepsilon}(\gamma(1))<0\right\}
$$

the Mountain Pass level, and with

$$
m_{\varepsilon}:=\inf _{u \in \mathcal{N}_{\varepsilon}} I_{\varepsilon}(u)
$$

the ground state level, it holds, in a standard way, that

$$
c_{\varepsilon}=m_{\varepsilon}=\inf _{u \in W \varepsilon \backslash\{0\}} \sup _{t \geq 0} I_{\varepsilon}(t u) .
$$

It is known that for "perturbed" problems a major role is played by the problem at infinity that we now introduce. 


\subsubsection{The problem at "infinity"}

Let us consider the "limit" problem (the autonomous problem) associated to $\left(P_{\varepsilon}^{*}\right)$, that is

$$
\left\{\begin{array}{l}
(-\Delta)^{s} u+\mu u=f(u) \\
u \in H^{s}\left(\mathbb{R}^{N}\right)
\end{array}\right.
$$

where $\mu>0$ is a constant. The solutions are critical points of the functional

$$
E_{\mu}(u)=\frac{1}{2} \int_{\mathbb{R}^{N}}\left|(-\Delta)^{s / 2} u\right|^{2}+\frac{\mu}{2} \int_{\mathbb{R}^{N}} u^{2}-\int_{\mathbb{R}^{N}} F(u) .
$$

in $H^{s}\left(\mathbb{R}^{N}\right)$. Denoting with $H_{\mu}^{s}\left(\mathbb{R}^{N}\right)$ simply the space $H^{s}\left(\mathbb{R}^{N}\right)$ endowed with the (equivalent squared) norm

$$
\|u\|_{H_{\mu}^{s}}^{2}:=\left|(-\Delta)^{s / 2} u\right|_{2}^{2}+\mu|u|_{2}^{2},
$$

by the assumptions of the nonlinearity $f$, it is easy to see that the functional $E_{\mu}$ has the Mountain Pass geometry with Mountain Pass level

$$
c_{\mu}^{\infty}:=\inf _{\gamma \in \mathcal{H}_{\mu}} \sup _{t \in[0,1]} E_{\mu}(\gamma(t)), \quad \mathcal{H}_{\mu}:=\left\{\gamma \in C\left([0,1], H_{\mu}^{s}\left(\mathbb{R}^{N}\right)\right): \gamma(0)=0, E_{\mu}(\gamma(1))<0\right\} .
$$

Introducing the set

$$
\mathcal{M}_{\mu}:=\left\{u \in H^{s}\left(\mathbb{R}^{N}\right) \backslash\{0\}:\|u\|_{H_{\mu}^{s}}^{2}=\int_{\mathbb{R}^{N}} f(u) u\right\}
$$

it is standard to see that

- $\mathcal{M}_{\mu}$ has a structure of differentiable manifold (said the Nehari manifold associated to $E_{\mu}$ ),

- $\mathcal{M}_{\mu}$ is bounded away from zero and radially homeomorphic to the set

$$
\mathcal{S}_{\mu}=\left\{u \in H_{\mu}^{s}\left(\mathbb{R}^{N}\right):\|u\|_{H_{\mu}^{s}}=1\right\} \backslash\left\{u \in H_{\mu}^{s}\left(\mathbb{R}^{N}\right): u \leq 0 \text { a.e. }\right\} .
$$

- the mountain pass value $c_{\mu}^{\infty}$ coincides with the ground state level

$$
m_{\mu}^{\infty}:=\inf _{u \in \mathcal{M}_{\mu}} E_{\mu}(u)>0
$$

The symbol " $\infty$ " in the notations is just to recall we are dealing with the limit problem. In the sequel we will mainly deal with $\mu=V_{0}$ and $\mu=V_{\infty}$ (whenever this last one is finite). Of course the inequality

$$
m_{\varepsilon} \geq m_{V_{0}}^{\infty}
$$

holds.

\subsection{Compactness properties for $I_{\varepsilon}, E_{\mu}$ : existence of a ground state solution}

We begin by showing the boundedness of the Palais-Smale sequences for $E_{\mu}$ in $H_{\mu}^{s}\left(\mathbb{R}^{N}\right)$ and $I_{\varepsilon}$ in $W_{\varepsilon}$. Let $\left\{u_{n}\right\} \subset H_{\mu}^{s}\left(\mathbb{R}^{N}\right)$ be a Palais-Smale sequence for $E_{\mu}$, that is, $\left|E_{\mu}\left(u_{n}\right)\right| \leq C$ and $E_{\mu}^{\prime}\left(u_{n}\right) \rightarrow 0$. Then, for large $n$,

$$
\begin{aligned}
C+\left\|u_{n}\right\|_{H_{\mu}^{s}}>E_{\mu}\left(u_{n}\right)-\frac{1}{K} E_{\mu}^{\prime}\left(u_{n}\right)\left[u_{n}\right] & =\left(\frac{1}{2}-\frac{1}{K}\right)\left\|u_{n}\right\|_{H_{\mu}^{s}}^{2}+\frac{1}{K} \int_{\mathbb{R}^{N}}\left(f\left(u_{n}\right) u_{n}-K F\left(u_{n}\right)\right) \\
& \geq\left(\frac{1}{2}-\frac{1}{K}\right)\left\|u_{n}\right\|_{H_{\mu}^{s}}^{2},
\end{aligned}
$$


and thus $\left\{u_{n}\right\}$ is bounded. Similarly we conclude for $I_{\varepsilon}$, using that

$$
\begin{aligned}
I_{\varepsilon}\left(u_{n}\right)-\frac{1}{K} I_{\varepsilon}^{\prime}\left(u_{n}\right)\left[u_{n}\right] & =\left(\frac{1}{2}-\frac{1}{K}\right)\left\|u_{n}\right\|_{\varepsilon}^{2}+\left(\frac{1}{4}-\frac{1}{K}\right) \int_{\mathbb{R}^{N}} \phi_{\varepsilon, u_{n}} u_{n}^{2}+\frac{1}{K} \int_{\mathbb{R}^{N}}\left(f\left(u_{n}\right) u_{n}-K F\left(u_{n}\right)\right) \\
& \geq\left(\frac{1}{2}-\frac{1}{K}\right)\left\|u_{n}\right\|_{\varepsilon}^{2} .
\end{aligned}
$$

In order to prove compactness, some preliminary work is needed. Let us recall the following Lions type lemma, whose proof can be found in d'Avenia et al. (2015, Lemma 2.3).

Lemma 2.3.1. If $\left\{u_{n}\right\}$ is bounded in $H^{s}\left(\mathbb{R}^{N}\right)$ and for some $R>0$ and $2 \leq r<2_{s}^{*}$ we have

$$
\sup _{x \in \mathbb{R}^{N}} \int_{B_{R}(x)}\left|u_{n}\right|^{r} \rightarrow 0 \quad \text { as } \quad n \rightarrow \infty,
$$

then $u_{n} \rightarrow 0$ in $L^{p}\left(\mathbb{R}^{N}\right)$ for $2<p<2_{s}^{*}$.

Then we can prove the following

Lemma 2.3.2. Let $\left\{u_{n}\right\} \subset W_{\varepsilon}$ be bounded and such that $I_{\varepsilon}^{\prime}\left(u_{n}\right) \rightarrow 0$. Then we have either

a) $u_{n} \rightarrow 0$ in $W_{\varepsilon}$, or

b) there exist a sequence $\left\{y_{n}\right\} \subset \mathbb{R}^{N}$ and constants $R, c>0$ such that

$$
\liminf _{n \rightarrow+\infty} \int_{B_{R}\left(y_{n}\right)} u_{n}^{2} \geq c>0 .
$$

Proof. Suppose that b) does not occur. Using Lemma 2.3.1 it follows

$$
u_{n} \rightarrow 0 \text { in } L^{p}\left(\mathbb{R}^{N}\right) \text { for } p \in\left(2,2_{s}^{*}\right) .
$$

Using (2.2.1), the boundedness of $\left\{u_{n}\right\}$ in $L^{2}\left(\mathbb{R}^{N}\right)$ and the fact that $u_{n} \rightarrow 0$ in $L^{q_{0}+1}\left(\mathbb{R}^{N}\right)$, we conclude that

$$
\int_{\mathbb{R}^{N}} f\left(u_{n}\right) u_{n} \rightarrow 0 .
$$

Finally, since

$$
\left\|u_{n}\right\|_{\varepsilon}^{2}-\int_{\mathbb{R}^{N}} f\left(u_{n}\right) u_{n} \leq\left\|u_{n}\right\|_{\varepsilon}^{2}+\int_{\mathbb{R}^{N}} \phi_{\varepsilon, u_{n}} u_{n}^{2}-\int_{\mathbb{R}^{N}} f\left(u_{n}\right) u_{n}=I_{\varepsilon}^{\prime}\left(u_{n}\right)\left[u_{n}\right]=o_{n}(1),
$$

it follows that $u_{n} \rightarrow 0$ in $W_{\varepsilon}$.

In the rest of the chapter we assume, without loss of generality, that $0 \in M$, that is, $V(0)=V_{0}$.

Lemma 2.3.3. Assume that $V_{\infty}<\infty$ and let $\left\{v_{n}\right\} \subset W_{\varepsilon}$ be a $(P S)_{d}$ sequence for $I_{\varepsilon}$ such that $v_{n} \rightarrow 0$ in $W_{\varepsilon}$. Then

$$
v_{n} \not \supset 0 \text { in } W_{\varepsilon} \Longrightarrow d \geq m_{V_{\infty}}^{\infty}
$$

Proof. Observe, preliminarly, that by condition (V1) it follows that

$$
\forall \xi>0 \exists \widetilde{R}=\widetilde{R}_{\xi}>0: \quad V(\varepsilon x)>V_{\infty}-\xi, \quad \forall x \notin B_{\widetilde{R}} .
$$

Let $\left\{t_{n}\right\} \subset(0,+\infty)$ be such that $\left\{t_{n} v_{n}\right\} \subset \mathcal{M}_{V_{\infty}}$. We start by showing the following

Claim: The sequence $\left\{t_{n}\right\}$ satisfies $\lim \sup _{n \rightarrow \infty} t_{n} \leq 1$. 
Supposing by contradiction that the claim does not hold, there exists $\delta>0$ and a subsequence still denoted by $\left\{t_{n}\right\}$, such that

$$
t_{n} \geq 1+\delta \text { for all } n \in \mathbb{N} \text {. }
$$

Since $\left\{v_{n}\right\}$ is a bounded $(P S)_{d}$ sequence for $I_{\varepsilon}, I_{\varepsilon}^{\prime}\left(v_{n}\right)\left[v_{n}\right]=o_{n}(1)$, that is,

$$
\left\|v_{n}\right\|_{\varepsilon}^{2}+\int_{\mathbb{R}^{N}} \phi_{\varepsilon, v_{n}} v_{n}^{2}=\int_{\mathbb{R}^{N}} f\left(v_{n}\right) v_{n}+o_{n}(1) .
$$

Moreover, since $\left\{t_{n} v_{n}\right\} \subset \mathcal{M}_{V_{\infty}}$, we get

$$
\left\|t_{n} v_{n}\right\|_{H_{V_{\infty}}^{s}}^{2}=\int_{\mathbb{R}^{N}} f\left(t_{n} v_{n}\right) t_{n} v_{n}
$$

These equalities imply that

$$
\int_{\mathbb{R}^{N}}\left(\frac{f\left(t_{n} v_{n}\right)}{t_{n}}-f\left(v_{n}\right)\right) v_{n}=\int_{\mathbb{R}^{N}}\left(V_{\infty}-V(\varepsilon x)\right) v_{n}^{2}-\int_{\mathbb{R}^{N}} \phi_{\varepsilon, v_{n}} v_{n}^{2}+o_{n}(1),
$$

and thus

$$
\int_{\mathbb{R}^{N}}\left(\frac{f\left(t_{n} v_{n}\right)}{t_{n}}-f\left(v_{n}\right)\right) v_{n} \leq \int_{\mathbb{R}^{N}}\left(V_{\infty}-V(\varepsilon x)\right) v_{n}^{2}+o_{n}(1) .
$$

Using (2.3.1), the fact that $v_{n} \rightarrow 0$ in $L^{2}\left(B_{\widetilde{R}}\right)$ and that $\left\{v_{n}\right\}$ is bounded in $W_{\varepsilon}$, let us say by some constant $C>0$, we deduce by $(2.3 .3)$

$$
\forall \xi>0: \int_{\mathbb{R}^{N}}\left(\frac{f\left(t_{n} v_{n}\right)}{t_{n}}-f\left(v_{n}\right)\right) v_{n} \leq \xi C+o_{n}(1) .
$$

Since $v_{n} \nrightarrow \nrightarrow 0$ in $W_{\varepsilon}$, we may invoke Lemma 2.3.2 to obtain $\left\{y_{n}\right\} \subset \mathbb{R}^{N}$ and $R, c>0$ such that

$$
\int_{B_{R}\left(y_{n}\right)} v_{n}^{2} \geq c
$$

Defining $\check{v}_{n}:=v_{n}\left(\cdot+y_{n}\right)$, we may suppose that, up to a subsequence,

$$
\check{v}_{n} \rightarrow \check{v} \text { in } H^{s}\left(\mathbb{R}^{N}\right)
$$

and, in view of (2.3.5), there exists a subset $\Omega \subset \mathbb{R}^{N}$ with positive measure such that $\check{v}>0$ in $\Omega$. By (f5) and (2.3.2), (2.3.4) becomes, for large $n$,

$$
0 \leq \int_{\Omega}\left(\frac{f\left((1+\delta) \check{v}_{n}\right)}{1+\delta}-f\left(\check{v}_{n}\right)\right) \check{v}_{n} \leq \xi C+o_{n}(1) .
$$

Now passing to the limit and applying Fatou's Lemma, it follows that, for every $\xi>0$

$$
0<\int_{\Omega}\left[\frac{f((1+\delta) \check{v})}{(1+\delta) \check{v}}-\frac{f(\check{v})}{\check{v}}\right] \check{v}^{2} \leq \xi C,
$$

which is absurd and proves the claim.

Now we distinguish two cases.

Case 1: $\limsup _{n \rightarrow \infty} t_{n}=1$.

Up to a subsequence we can assume that $t_{n} \rightarrow 1$. We have,

$$
d+o_{n}(1)=I_{\varepsilon}\left(v_{n}\right) \geq m_{V_{\infty}}^{\infty}+I_{\varepsilon}\left(v_{n}\right)-E_{V_{\infty}}\left(t_{n} v_{n}\right) .
$$


Moreover,

$$
\begin{aligned}
I_{\varepsilon}\left(v_{n}\right)-E_{V_{\infty}}\left(t_{n} v_{n}\right)= & \frac{\left(1-t_{n}^{2}\right)}{2} \int_{\mathbb{R}^{N}}\left|(-\Delta)^{s / 2} v_{n}\right|^{2}+\frac{1}{2} \int_{\mathbb{R}^{N}}\left(V(\varepsilon x)-t_{n}^{2} V_{\infty}\right) v_{n}^{2} \\
& +\frac{1}{4} \int_{\mathbb{R}^{n}} \phi_{\varepsilon, v_{n}} v_{n}^{2}+\int_{\mathbb{R}^{N}}\left(F\left(t_{n} v_{n}\right)-F\left(v_{n}\right)\right),
\end{aligned}
$$

and due to the boundedness of $\left\{v_{n}\right\}$ we get, for every $\xi>0$,

$$
I_{\varepsilon}\left(v_{n}\right)-E_{V_{\infty}}\left(t_{n} v_{n}\right) \geq o_{n}(1)-C \xi+\int_{\mathbb{R}^{N}}\left(F\left(t_{n} v_{n}\right)-F\left(v_{n}\right)\right),
$$

where we have used again (2.3.1). By the Mean Value Theorem, $\int_{\mathbb{R}^{N}}\left(F\left(t_{n} v_{n}\right)-F\left(v_{n}\right)\right)=o_{n}(1)$, therefore (2.3.6) becomes

$$
d+o_{n}(1) \geq m_{V_{\infty}}^{\infty}-C \xi+o_{n}(1),
$$

and taking the limit in $n$, by the arbitrariness of $\xi$, we deduce $d \geq m_{V_{\infty}}^{\infty}$.

Case 2: $\limsup _{n \rightarrow \infty} t_{n}=t_{0}<1$.

We can assume $t_{n} \rightarrow t_{0}$ and $t_{n}<1$. Since $t \mapsto \frac{1}{4} f(t) t-F(t)$ is increasing in $(0, \infty)$,

$$
\begin{aligned}
m_{V_{\infty}}^{\infty} \leq E_{V_{\infty}}\left(t_{n} v_{n}\right) & =\int_{\mathbb{R}^{N}}\left(\frac{1}{2} f\left(t_{n} v_{n}\right) t_{n} v_{n}-F\left(t_{n} v_{n}\right)\right) \\
& =\int_{\mathbb{R}^{N}} \frac{1}{4} f\left(t_{n} v_{n}\right) t_{n} v_{n}+\int_{\mathbb{R}^{N}}\left(\frac{1}{4} f\left(t_{n} v_{n}\right) t_{n} v_{n}-F\left(t_{n} v_{n}\right)\right) \\
& =\frac{1}{4}\left\|t_{n} v_{n}\right\|_{H_{V_{\infty}}^{s}}^{2}+\int_{\mathbb{R}^{N}}\left(\frac{1}{4} f\left(t_{n} v_{n}\right) t_{n} v_{n}-F\left(t_{n} v_{n}\right)\right) \\
& \leq \frac{1}{4}\left\|t_{n} v_{n}\right\|_{H_{V_{\infty}}^{s}}^{2}+\int_{\mathbb{R}^{N}}\left(\frac{1}{4} f\left(v_{n}\right) v_{n}-F\left(v_{n}\right)\right) .
\end{aligned}
$$

But

$$
\left\|t_{n} v_{n}\right\|_{H_{V_{\infty}}^{s}}^{2} \leq \int_{\mathbb{R}^{N}}\left|(-\Delta)^{s / 2} v_{n}\right|^{2}+\int_{\mathbb{R}^{N}} t_{n}^{2} V_{\infty} v_{n}^{2}
$$

Again by (2.3.1), given $\xi>0$,

$$
t_{n}^{2} V_{\infty}-\xi<V_{\infty}-\xi<V(\varepsilon x) \quad \text { for } x \notin B_{\widetilde{R}}
$$

and hence

$$
\begin{aligned}
\int_{\mathbb{R}^{N}} t_{n}^{2} V_{\infty} v_{n}^{2} & \leq \int_{B_{\widetilde{R}}} V_{\infty} v_{n}^{2}+\int_{|x| \geq \widetilde{R}} V(\varepsilon x) v_{n}^{2}+\int_{|x| \geq \widetilde{R}} \xi v_{n}^{2} \\
& \leq o_{n}(1)+\int_{\mathbb{R}^{N}} V(\varepsilon x) v_{n}^{2}+C \xi .
\end{aligned}
$$

From this and (2.3.8) we have

$$
\left\|t_{n} v_{n}\right\|_{H_{V_{\infty}}^{s}}^{2} \leq\left\|v_{n}\right\|_{\varepsilon}^{2}+C \xi+o_{n}(1)
$$


Therefore, using (2.3.7)

$$
\begin{aligned}
m_{V_{\infty}}^{\infty} & \leq \frac{1}{4}\left\|v_{n}\right\|_{\varepsilon}^{2}+\int_{\mathbb{R}^{N}}\left(\frac{1}{4} f\left(v_{n}\right) v_{n}-F\left(v_{n}\right)\right)+C \xi+o_{n}(1) \\
& =I_{\varepsilon}\left(v_{n}\right)-\frac{1}{4} I_{\varepsilon}^{\prime}\left(v_{n}\right)\left[v_{n}\right]+C \xi+o_{n}(1) \\
& =d+C \xi+o_{n}(1),
\end{aligned}
$$

concluding the proof.

Proposition 2.3.4. The functional $I_{\varepsilon}$ in $W_{\varepsilon}$ satisfies the $(P S)_{c}$ condition

1. at any level $c<m_{V_{\infty}}^{\infty}$, if $V_{\infty}<\infty$,

2. at any level $c \in \mathbb{R}$, if $V_{\infty}=\infty$.

Proof. Let $\left\{u_{n}\right\} \subset W_{\varepsilon}$ be such that $I_{\varepsilon}\left(u_{n}\right) \rightarrow c$ and $I_{\varepsilon}^{\prime}\left(u_{n}\right) \rightarrow 0$. We have already seen that $\left\{u_{n}\right\}$ is bounded in $W_{\varepsilon}$. Thus there exists $u \in W_{\varepsilon}$ such that, up to a subsequence, $u_{n} \rightarrow u$ in $W_{\varepsilon}$. Note that $I_{\varepsilon}^{\prime}(u)=0$, since by Lemma 2.2.1 (iv), we have for every $w \in W_{\varepsilon}$

$$
\left(u_{n}, w\right)_{\varepsilon} \rightarrow(u, w)_{\varepsilon}, \quad A^{\prime}\left(u_{n}\right)[w] \rightarrow A^{\prime}(u)[w] \quad \text { and } \int_{\mathbb{R}^{N}} f\left(u_{n}\right) w \rightarrow \int_{\mathbb{R}^{N}} f(u) w .
$$

Defining $v_{n}:=u_{n}-u$, we have that $\int_{\mathbb{R}^{N}} F\left(v_{n}\right)=\int_{\mathbb{R}^{N}} F\left(u_{n}\right)-\int_{\mathbb{R}^{N}} F(u)+o_{n}(1)$ (see Alves et al. (2004)) and by Lemma 2.2.1 (vi), we have $A\left(v_{n}\right)=A\left(u_{n}\right)-A(u)+o_{n}(1)$; hence arguing as in Alves and Figueiredo (2006), we obtain also

$$
I_{\varepsilon}^{\prime}\left(v_{n}\right) \rightarrow 0
$$

Moreover

$$
I_{\varepsilon}\left(v_{n}\right)=I_{\varepsilon}\left(u_{n}\right)-I_{\varepsilon}(u)+o_{n}(1)=c-I_{\varepsilon}(u)+o_{n}(1)=: d+o_{n}(1)
$$

and (2.3.9) and (2.3.10) show that $\left\{v_{n}\right\}$ is a $(P S)_{d}$ sequence. By (f4),

$$
\begin{aligned}
I_{\varepsilon}(u)=I_{\varepsilon}(u)-\frac{1}{4} I_{\varepsilon}^{\prime}(u)[u]=\frac{1}{4}\|u\|_{\varepsilon}^{2}+\int_{\mathbb{R}^{N}}\left(\frac{1}{4} f(u) u-F(u)\right) & \\
& \geq \frac{1}{4} \int_{\mathbb{R}^{N}}(f(u) u-4 F(u)) \geq 0
\end{aligned}
$$

and then coming back in (2.3.10) we have

$$
d \leq c
$$

Then,

1. if $V_{\infty}<\infty$, and $c<m_{V_{\infty}}^{\infty}$, by (2.3.11) we obtain

$$
d \leq c<m_{V_{\infty}}^{\infty} .
$$

It follows from Lemma 2.3.3 that $v_{n} \rightarrow 0$, that is $u_{n} \rightarrow u$ in $W_{\varepsilon}$.

2. If $V_{\infty}=\infty$, by the compact imbedding $W_{\varepsilon} \hookrightarrow \hookrightarrow L^{r}\left(\mathbb{R}^{N}\right), 2 \leq r<2_{s}^{*}$, up to a subsequence, $v_{n} \rightarrow 0$ in $L^{r}\left(\mathbb{R}^{N}\right)$ and since $I_{\varepsilon}^{\prime}\left(v_{n}\right) \rightarrow 0$, we have

$$
I_{\varepsilon}^{\prime}\left(v_{n}\right)\left[v_{n}\right]=\left\|v_{n}\right\|_{\varepsilon}^{2}+\int_{\mathbb{R}^{N}} \phi_{\varepsilon, v_{n}} v_{n}^{2}-\int_{\mathbb{R}^{N}} f\left(v_{n}\right) v_{n}=o_{n}(1)
$$

By Lemma 2.2.1 $(v)$,

$$
A\left(v_{n}\right)=\int_{\mathbb{R}^{N}} \phi_{\varepsilon, v_{n}} v_{n}^{2}=o_{n}(1),
$$


and since by (2.2.1) it holds again $\int_{\mathbb{R}^{N}} f\left(v_{n}\right) v_{n}=o_{n}(1)$, we have by $(2.3 .12)\left\|v_{n}\right\|_{\varepsilon}^{2}=o_{n}(1)$, that is $u_{n} \rightarrow u$ in $W_{\varepsilon}$.

The proof is thereby complete.

As a consequence it is standard to prove that

Proposition 2.3.5. The functional $I_{\varepsilon}$ restricted to $\mathcal{N}_{\varepsilon}$ satisfies the $(P S)_{c}$ condition

1. at any level $c<m_{V_{\infty}}^{\infty}$, if $V_{\infty}<\infty$,

2. at any level $c \in \mathbb{R}$, if $V_{\infty}=\infty$.

Moreover, the constrained critical points of the functional $I_{\varepsilon}$ on $\mathcal{N}_{\varepsilon}$ are critical points of $I_{\varepsilon}$ in $W_{\varepsilon}$, hence solutions of $\left(P_{\varepsilon}^{*}\right)$.

Let us recall the following result (see Figueiredo and Siciliano 2016, Lemma 6) concerning problem $\left(A_{\mu}\right)$.

Lemma 2.3.6 (Ground state for the autonomous problem). Let $\left\{u_{n}\right\} \subset \mathcal{M}_{\mu}$ be a sequence satisfying $E_{\mu}\left(u_{n}\right) \rightarrow m_{\mu}^{\infty}$. Then, up to subsequences the following alternative holds:

a) $\left\{u_{n}\right\}$ strongly converges in $H^{s}\left(\mathbb{R}^{N}\right)$;

b) there exists a sequence $\left\{\tilde{y}_{n}\right\} \subset \mathbb{R}^{N}$ such that $u_{n}\left(\cdot+\tilde{y}_{n}\right)$ strongly converges in $H^{s}\left(\mathbb{R}^{N}\right)$.

In particular, there exists a minimizer $\mathfrak{w}_{\mu}>0$ for $m_{\mu}^{\infty}$.

Now we can prove the existence of a ground state for our problem. Assumption (H1) is tacitly assumed.

Theorem 2.3.7. Suppose that $f$ verifies (f1)-(f5) and $V$ verifies (V1). Then there exists a ground state solution $\mathfrak{u}_{\varepsilon} \in W_{\varepsilon}$ of $\left(P_{\varepsilon}^{*}\right)$,

1. for every $\varepsilon \in(0, \bar{\varepsilon}]$, for some $\bar{\varepsilon}>0$, if $V_{\infty}<\infty$;

2. for every $\varepsilon>0$, if $V_{\infty}=\infty$.

Proof. Since the functional $I_{\varepsilon}$ has the geometry of the Mountain Pass Theorem in $W_{\varepsilon}$ there exists $\left\{u_{n}\right\} \subset W_{\varepsilon}$ satisfying

$$
I_{\varepsilon}\left(u_{n}\right) \rightarrow c_{\varepsilon} \text { and } I_{\varepsilon}^{\prime}\left(u_{n}\right) \rightarrow 0
$$

1. If $V_{\infty}<\infty$, in virtue of Proposition 2.3.4, we have only to show that $c_{\varepsilon}<m_{V_{\infty}}^{\infty}$ for every positive $\varepsilon$ smaller than a certain $\bar{\varepsilon}$.

Let $\mu \in\left(V_{0}, V_{\infty}\right)$, so that

$$
m_{V_{0}}^{\infty}<m_{\mu}^{\infty}<m_{V_{\infty}}^{\infty}
$$

For $r>0$ let $\eta_{r}$ be a smooth cut-off function in $\mathbb{R}^{N}$ which equals 1 on $B_{r}$ and with support in $B_{2 r}$. Let $w_{r}:=\eta_{r} \mathfrak{w}_{\mu}$ and $s_{r}>0$ such that $s_{r} w_{r} \in \mathcal{M}_{\mu}$. If it were, for every $r>0: E_{\mu}\left(s_{r} w_{r}\right) \geq m_{V_{\infty}}^{\infty}$, since $w_{r} \rightarrow \mathfrak{w}_{\mu}$ in $H^{s}\left(\mathbb{R}^{N}\right)$ for $r \rightarrow+\infty$, we would have $s_{r} \rightarrow 1$ and then

$$
m_{V_{\infty}}^{\infty} \leq \liminf _{r \rightarrow+\infty} E_{\mu}\left(s_{r} w_{r}\right)=E_{\mu}\left(\mathfrak{w}_{\mu}\right)=m_{\mu}^{\infty}
$$

which contradicts (2.3.13). This means that there exists $\bar{r}>0$ such that $\omega:=s_{\bar{r}} w_{\bar{r}} \in \mathcal{M}_{\mu}$ satisfies

$$
E_{\mu}(\omega)<m_{V_{\infty}}^{\infty} .
$$


Given $\varepsilon>0$, let $t_{\varepsilon}>0$ the number such that $t_{\varepsilon} \omega \in \mathcal{N}_{\varepsilon}$. Therefore

$$
t_{\varepsilon}^{2}\|\omega\|_{\varepsilon}^{2}+t_{\varepsilon}^{4} \int_{\mathbb{R}^{N}} \phi_{\varepsilon, \omega} \omega^{2}=t_{\varepsilon} \int_{\mathbb{R}^{N}} f\left(t_{\varepsilon} \omega\right) \omega
$$

implying that

$$
\frac{\|\omega\|_{\varepsilon}^{2}}{t_{\varepsilon}^{2}}+\int_{\mathbb{R}^{N}} \phi_{\varepsilon, \omega} \omega^{2} \geq \int_{B_{\bar{r}}} \frac{f\left(t_{\varepsilon} \omega\right)}{\left(t_{\varepsilon} \omega\right)^{3}} \omega^{4}
$$

Now we claim that there exists $T>0$ such that $\lim _{\sup _{\varepsilon \rightarrow 0^{+}}} t_{\varepsilon} \leq T$. If by contradiction there exists $\varepsilon_{n} \rightarrow 0^{+}$with $t_{\varepsilon_{n}} \rightarrow \infty$, then by (2.3.15) and (f5) we have

$$
\frac{\|\omega\|_{\varepsilon_{n}}^{2}}{t_{\varepsilon_{n}}^{2}}+\int_{\mathbb{R}^{N}} \phi_{\varepsilon_{n}, \omega} \omega^{2} \geq \frac{f\left(t_{\varepsilon_{n}} \omega(\bar{x})\right)}{\left(t_{\varepsilon_{n}} \omega(\bar{x})\right)^{3}} \int_{B_{\bar{r}}} \omega^{4}
$$

where $\omega(\bar{x}):=\min _{B_{\bar{r}}} \omega(x)>0$. The absurd is achieved by passing to the limit in $n$, since by (f5) the right hand side of (2.3.16) tends to $\infty$, while the left hand side tends to 0 .

Then there exists $\varepsilon_{1}>0$ such that

$$
\forall \varepsilon \in\left(0, \varepsilon_{1}\right]: \quad t_{\varepsilon} \in(0, T] .
$$

Condition (V1) implies also that there exists some $\varepsilon_{2}>0$ such that

$$
\forall \varepsilon \in\left(0, \varepsilon_{2}\right]: \quad V(\varepsilon x) \leq \frac{V_{0}+\mu}{2}, \text { for all } x \in \operatorname{supp} \omega .
$$

Finally let

$$
\varepsilon_{3}:=\left(\frac{\left(\mu-V_{0}\right)|\omega|_{2}^{2}}{C_{\mathrm{e}} T^{2}\|\omega\|^{4}}\right)^{1 /(\alpha-\theta)},
$$

where $C_{\mathrm{e}}$ is the same constant appearing in (2.2.5), hence in particular

$$
\forall \varepsilon \in\left(0, \varepsilon_{3}\right]: \quad \int_{\mathbb{R}^{N}} \phi_{\varepsilon, \omega} \omega^{2} \leq \varepsilon^{\alpha-\theta} C_{\mathrm{e}}\|\omega\|^{4} \quad \text { and } \quad T^{2} \varepsilon^{\alpha-\theta} C_{\mathrm{e}}\|\omega\|^{4} \leq\left(\mu-V_{0}\right) \int_{\mathbb{R}^{N}} \omega^{2} .
$$

Let $\bar{\varepsilon}:=\min \left\{\varepsilon_{1}, \varepsilon_{2}, \varepsilon_{3}\right\}$. By using (2.3.17)-(2.3.18) we have, for every $\varepsilon \in(0, \bar{\varepsilon}]$ :

$$
\int_{\mathbb{R}^{N}} V(\varepsilon x) \omega^{2}+\frac{t_{\varepsilon}^{2}}{2} \int_{\mathbb{R}^{N}} \phi_{\varepsilon, \omega} \omega^{2} \leq \frac{V_{0}+\mu}{2}|\omega|_{2}^{2}+\frac{1}{2} T^{2} \varepsilon^{\alpha-\theta} C_{\mathrm{e}}\|\omega\|^{4} \leq \mu \int_{\mathbb{R}^{N}} \omega^{2},
$$

from which we infer $I_{\varepsilon}\left(t_{\varepsilon} \omega\right) \leq E_{\mu}\left(t_{\varepsilon} \omega\right)$. Then by (2.2.6) and (2.3.14),

$$
c_{\varepsilon} \leq I_{\varepsilon}\left(t_{\varepsilon} \omega\right) \leq E_{\mu}\left(t_{\varepsilon} \omega\right) \leq E_{\mu}(\omega)<m_{V_{\infty}}^{\infty} .
$$

which concludes the proof in this case.

2. If $V_{\infty}=\infty$, by Proposition 2.3.4, $\left\{u_{n}\right\}$ strongly converges to some $\mathfrak{u}_{\varepsilon}$ in $H^{s}\left(\mathbb{R}^{N}\right)$, which satisfies

$$
I_{\varepsilon}\left(\mathfrak{u}_{\varepsilon}\right)=c_{\varepsilon} \text { and } I_{\varepsilon}^{\prime}\left(\mathfrak{u}_{\varepsilon}\right)=0 .
$$

and $\mathfrak{u}_{\varepsilon}$ is the ground state we were looking for.

\subsection{Proof of Theorem 2.1.1}

In this section we introduce the barycenter map in order to study the "topological complexity" of suitable sublevels of the functional $I_{\varepsilon}$ in the Nehari manifold. Let us start with the following

Proposition 2.4.1. Let $\varepsilon_{n} \rightarrow 0^{+}$and $u_{n} \in \mathcal{N}_{\varepsilon_{n}}$ be such that $I_{\varepsilon_{n}}\left(u_{n}\right) \rightarrow m_{V_{0}}^{\infty}$. Then there exists a 
sequence $\left\{\tilde{y}_{n}\right\} \subset \mathbb{R}^{N}$ such that $u_{n}\left(\cdot+\tilde{y}_{n}\right)$ has a convergent subsequence in $H^{s}\left(\mathbb{R}^{N}\right)$. Moreover, up to a subsequence, $y_{n}:=\varepsilon_{n} \tilde{y}_{n} \rightarrow y \in M$.

Recall that $M$ is the set where $V$ achieves the minimum $V_{0}$.

Proof. We begin by showing that $\left\{u_{n}\right\}$ is bounded in $H_{V_{0}}^{s}\left(\mathbb{R}^{N}\right)$. By assumptions, $I_{\varepsilon_{n}}^{\prime}\left(u_{n}\right)\left[u_{n}\right]=0$ and $I_{\varepsilon_{n}}\left(u_{n}\right) \rightarrow m_{V_{0}}^{\infty}$ write as

$$
\left\|u_{n}\right\|_{\varepsilon_{n}}^{2}+\int_{\mathbb{R}^{N}} \phi_{\varepsilon_{n}, u_{n}} u_{n}^{2}=\int_{\mathbb{R}^{N}} f\left(u_{n}\right) u_{n}
$$

and

$$
\frac{1}{2}\left\|u_{n}\right\|_{\varepsilon_{n}}^{2}+\frac{1}{4} \int_{\mathbb{R}^{N}} \phi_{\varepsilon_{n}, u_{n}} u_{n}^{2}-\int_{\mathbb{R}^{N}} F\left(u_{n}\right)=m_{V_{0}}^{\infty}+o_{n}(1)
$$

which combined together give

$$
\frac{1}{4} \int_{\mathbb{R}^{N}} f\left(u_{n}\right) u_{n}-\int_{\mathbb{R}^{N}} F\left(u_{n}\right)=\frac{1}{4}\left(\left\|u_{n}\right\|_{\varepsilon_{n}}^{2}+\int_{\mathbb{R}^{N}} \phi_{\varepsilon_{n}, u_{n}} u_{n}^{2}\right)-\int_{\mathbb{R}^{N}} F\left(u_{n}\right) \leq m_{V_{0}}^{\infty}+o_{n}(1) .
$$

Using (f4) we get

$$
0 \leq\left(\frac{1}{4}-\frac{1}{K}\right) \int_{\mathbb{R}^{N}} f\left(u_{n}\right) u_{n} \leq m_{V_{0}}^{\infty}+o_{n}(1),
$$

and therefore, coming back to (2.4.1), for some positive constant $C_{1}$ (independent on $n$ )

$$
\left\|u_{n}\right\|_{H_{V_{0}}^{s}} \leq\left\|u_{n}\right\|_{\varepsilon_{n}} \leq C_{1} .
$$

We prove the following

Claim: there exists $\left\{\tilde{y}_{n}\right\} \subset \mathbb{R}^{N}$ and $R, c>0$ such that $\liminf _{n \rightarrow \infty} \int_{B_{R}\left(\tilde{y}_{n}\right)} u_{n}^{2} \geq c>0$.

Indeed, if it were not the case then

$$
\lim _{n \rightarrow \infty} \sup _{y \in \mathbb{R}^{N}} \int_{B_{R}(y)} u_{n}^{2}=0, \quad \text { for every } R>0 .
$$

By Lemma 2.3.1, $u_{n} \rightarrow 0$ in $L^{p}\left(\mathbb{R}^{N}\right)$, for $2<p<2_{s}^{*}$ and then

$$
\int_{\mathbb{R}^{N}} f\left(u_{n}\right) u_{n} \rightarrow 0
$$

Therefore $\left\|u_{n}\right\|_{\varepsilon_{n}}^{2}+\int_{\mathbb{R}^{N}} \phi_{\varepsilon_{n}, u_{n}} u_{n}^{2}=o_{n}(1)$, and also from

$$
0 \leq \int_{\mathbb{R}^{N}} F\left(u_{n}\right) \leq \frac{1}{K} \int_{\mathbb{R}^{N}} f\left(u_{n}\right) u_{n}
$$

we have $\int_{\mathbb{R}^{N}} F\left(u_{n}\right)=o_{n}(1)$. But then $\lim _{n \rightarrow \infty} I_{\varepsilon_{n}}\left(u_{n}\right)=m_{V_{0}}^{\infty}=0$ which is a contradiction and proves our claim.

Then the sequence $v_{n}:=u_{n}\left(\cdot+\tilde{y}_{n}\right)$ is also bounded in $H^{s}\left(\mathbb{R}^{N}\right)$ and

$$
v_{n} \rightarrow v \not \equiv 0 \quad \text { in } \quad H^{s}\left(\mathbb{R}^{N}\right)
$$

since

$$
\int_{B_{R}} v^{2}=\liminf _{n \rightarrow \infty} \int_{B_{R}} v_{n}^{2}=\liminf _{n \rightarrow \infty} \int_{B_{R}\left(\tilde{y}_{n}\right)} u_{n}^{2} \geq c>0
$$

by the claim. 
Let now $t_{n}>0$ be such that $\tilde{v}_{n}:=t_{n} v_{n} \in \mathcal{M}_{V_{0}}$; the next step is to prove that

$$
E_{V_{0}}\left(\tilde{v}_{n}\right) \rightarrow m_{V_{0}}^{\infty}
$$

For this, note that

$$
\begin{aligned}
m_{V_{0}}^{\infty} & \leq E_{V_{0}}\left(\tilde{v}_{n}\right) \\
& =\frac{1}{2}\left\|\tilde{v}_{n}\right\|_{V_{0}}^{2}-\int_{\mathbb{R}^{N}} F\left(\tilde{v}_{n}\right) \\
& =\frac{t_{n}^{2}}{2} \int_{\mathbb{R}^{N}}\left[\left|(-\Delta)^{s / 2} u_{n}\left(x+\tilde{y}_{n}\right)\right|^{2}+V_{0} u_{n}^{2}\left(x+\tilde{y}_{n}\right)\right] d x-\int_{\mathbb{R}^{N}} F\left(t_{n} u_{n}\left(x+\tilde{y}_{n}\right)\right) d x \\
& =\frac{t_{n}^{2}}{2} \int_{\mathbb{R}^{N}}\left|(-\Delta)^{s / 2} u_{n}(z)\right|^{2} d z+\frac{t_{n}^{2}}{2} \int_{\mathbb{R}^{N}} V_{0} u_{n}^{2}(z) d z-\int_{\mathbb{R}^{N}} F\left(t_{n} u_{n}(z)\right) d z \\
& \leq \frac{t_{n}^{2}}{2} \int_{\mathbb{R}^{N}}\left|(-\Delta)^{s / 2} u_{n}\right|^{2}+\frac{t_{n}^{2}}{2} \int_{\mathbb{R}^{N}} V\left(\varepsilon_{n} z\right) u_{n}^{2}+\frac{t_{n}^{4}}{4} \int_{\mathbb{R}^{N}} \phi_{\varepsilon_{n}, u_{n}} u_{n}^{2}-\int_{\mathbb{R}^{N}} F\left(t_{n} u_{n}\right) \\
& =I_{\varepsilon_{n}}\left(t_{n} u_{n}\right)
\end{aligned}
$$

and then

$$
m_{V_{0}}^{\infty} \leq E_{V_{0}}\left(\tilde{v}_{n}\right) \leq I_{\varepsilon_{n}}\left(t_{n} u_{n}\right) \leq I_{\varepsilon_{n}}\left(u_{n}\right)=m_{V_{0}}^{\infty}+o_{n}(1)
$$

which proves (2.4.4).

We can prove now that $v_{n} \rightarrow v$ in $H^{s}\left(\mathbb{R}^{N}\right)$. As in the first part of the proof (where we proved the boundedness of $\left\{u_{n}\right\}$ in $\left.H_{V_{0}}^{s}\left(\mathbb{R}^{N}\right)\right)$, it is easy to see that

$$
\left\{\tilde{v}_{n}\right\} \subset \mathcal{M}_{V_{0}} \text { and } \quad E_{V_{0}}\left(\tilde{v}_{n}\right) \rightarrow m_{V_{0}}^{\infty} \Longrightarrow\left\|\tilde{v}_{n}\right\|_{H_{V_{0}}^{s}} \leq C,
$$

and an analogous claim as before holds for the sequence $\left\{\tilde{v}_{n}\right\}$. Then $\tilde{v}_{n} \rightarrow \bar{v}$ in $H_{V_{0}}^{s}\left(\mathbb{R}^{N}\right)$. Since $\left\|v_{n}\right\|_{H_{V_{0}}^{s}} \nrightarrow 0$, there exists $\delta>0$ such that

$$
0<\delta \leq\left\|v_{n}\right\|_{H_{V_{0}}^{s}}
$$

This implies

$$
0<t_{n} \delta \leq\left\|t_{n} v_{n}\right\|_{H_{V_{0}}^{s}}=\left\|\tilde{v}_{n}\right\|_{H_{V_{0}}^{s}} \leq C
$$

showing that, up to subsequence, $t_{n} \rightarrow t_{0} \geq 0$. If now $t_{0}=0$ using (2.4.2) we derive

$$
0 \leq\left\|\tilde{v}_{n}\right\|_{H_{V_{0}}^{s}}=t_{n}\left\|v_{n}\right\|_{H_{V_{0}}^{s}} \leq t_{n} C_{1} \rightarrow 0
$$

so that $\tilde{v}_{n} \rightarrow 0$ in $H_{V_{0}}^{s}\left(\mathbb{R}^{N}\right)$. From this and (2.4.4) it follows $m_{V_{0}}^{\infty}=0$ which is absurd. So $t_{0}>0$. Then $t_{n} v_{n} \rightarrow t_{0} \bar{v}=: \tilde{v}$ in $H^{s}\left(\mathbb{R}^{N}\right)$ and by (2.4.5) $\tilde{v} \not \equiv 0$. By Lemma 2.3.6 applied to $\left\{\tilde{v}_{n}\right\}$ we get $\tilde{v}_{n} \rightarrow \tilde{v}$ in $H^{s}\left(\mathbb{R}^{N}\right)$ and then $v_{n} \rightarrow \bar{v}$. By (2.4.3) we deduce $v_{n} \rightarrow v$ and the first part of the proposition is proved.

We proceed to prove the second part. We first state that $\left\{y_{n}\right\}$ is bounded in $\mathbb{R}^{N}$ (here $y_{n}=\varepsilon_{n} \tilde{y}_{n}$ with $\tilde{y}_{n}$ given in the above claim). Assume the contrary; then

1. if $V_{\infty}<\infty$, since $\tilde{v}_{n} \rightarrow \tilde{v}$ in $H^{s}\left(\mathbb{R}^{N}\right)$ and $V_{0}<V_{\infty}$, we have 


$$
\begin{aligned}
m_{V_{0}}^{\infty} & =\frac{1}{2}\|\tilde{v}\|_{H_{V_{0}}^{s}}^{2}-\int_{\mathbb{R}^{N}} F(\tilde{v})<\frac{1}{2}\|\tilde{v}\|_{H_{V_{\infty}}^{s}}^{2}-\int_{\mathbb{R}^{N}} F(\tilde{v}) \\
& \leq \liminf _{n \rightarrow \infty} \frac{1}{2} \int_{\mathbb{R}^{N}}\left|(-\Delta)^{s / 2} \tilde{v}_{n}\right|^{2}+\lim _{n \rightarrow \infty}\left(\frac{1}{2} \int_{\mathbb{R}^{N}} V\left(\varepsilon_{n} x+y_{n}\right) \tilde{v}_{n}^{2}(x) d x-\int_{\mathbb{R}^{N}} F\left(\tilde{v}_{n}\right)\right) \\
& =\liminf _{n \rightarrow \infty}\left(\frac{t_{n}^{2}}{2} \int_{\mathbb{R}^{N}}\left|(-\Delta)^{s / 2} u_{n}\right|^{2}+\frac{t_{n}^{2}}{2} \int_{\mathbb{R}^{N}} V\left(\varepsilon_{n} z\right) u_{n}^{2}-\int_{\mathbb{R}^{N}} F\left(t_{n} u_{n}\right)\right) \\
& \leq \liminf _{n \rightarrow \infty}\left(\frac{1}{2}\left\|t_{n} u_{n}\right\|_{\varepsilon_{n}}^{2}-\int_{\mathbb{R}^{N}} F\left(t_{n} u_{n}\right)+\frac{t_{n}^{4}}{4} \int_{\mathbb{R}^{N}} \phi_{\varepsilon_{n}, u_{n}} u_{n}^{2}\right)
\end{aligned}
$$

from which

$$
m_{V_{0}}^{\infty}<\liminf _{n \rightarrow \infty} I_{\varepsilon_{n}}\left(t_{n} u_{n}\right) \leq \liminf _{n \rightarrow \infty} I_{\varepsilon_{n}}\left(u_{n}\right)=m_{V_{0}}^{\infty}
$$

which is a contradiction.

2. If $V_{\infty}=\infty$, we have

$$
\begin{aligned}
\int_{\mathbb{R}^{N}} V\left(\varepsilon_{n} x+y_{n}\right) v_{n}^{2}(x) d x \leq & \int_{\mathbb{R}^{N}}\left|(-\Delta)^{s / 2} v_{n}(x)\right|^{2} d x+\int_{\mathbb{R}^{N}} V\left(\varepsilon_{n} x+y_{n}\right) v_{n}^{2}(x) d x \\
& +\int_{\mathbb{R}^{N}} \phi_{\varepsilon_{n}, v_{n}}(x) v_{n}^{2}(x) d x \\
& =\int_{\mathbb{R}^{N}} f\left(v_{n}(x)\right) v_{n}(x) d x
\end{aligned}
$$

and by the Fatou's Lemma we obtain the absurd

$$
\infty=\liminf _{n \rightarrow \infty} \int_{\mathbb{R}^{N}} f\left(v_{n}\right) v_{n}=\int_{\mathbb{R}^{N}} f(v) v .
$$

Then $\left\{y_{n}\right\}$ has to be bounded and we can assume $y_{n} \rightarrow y \in \mathbb{R}^{N}$. If $y \notin M$ then $V_{0}<V(y)$, and similarly to the computation made in case 1 . above (simply replace $V_{\infty}$ with $V(y)$ ) we have a contradiction. Hence $y \in M$ and the proof is thereby complete.

For $\delta>0$ (later on it will be fixed conveniently) let $\eta$ be a smooth nonincreasing cut-off function defined in $[0, \infty)$ such that

$$
\eta(t)= \begin{cases}1 & \text { if } 0 \leq t \leq \delta / 2 \\ 0 & \text { if } t \geq \delta\end{cases}
$$

Let $\mathfrak{w}_{V_{0}}$ be a ground state solution given in Lemma 2.3.6 of problem $\left(A_{\mu}\right)$ with $\mu=V_{0}$ and for any $y \in M$, let us define

$$
\Psi_{\varepsilon, y}(x):=\eta(|\varepsilon x-y|) \mathfrak{w}_{V_{0}}\left(\frac{\varepsilon x-y}{\varepsilon}\right) .
$$

Let $t_{\varepsilon}>0$ verifying $\max _{t \geq 0} I_{\varepsilon}\left(t \Psi_{\varepsilon, y}\right)=I_{\varepsilon}\left(t_{\varepsilon} \Psi_{\varepsilon, y}\right)$, so that $t_{\varepsilon} \Psi_{\varepsilon, y} \in \mathcal{N}_{\varepsilon}$, and let

$$
\Phi_{\varepsilon}: y \in M \mapsto t_{\varepsilon} \Psi_{\varepsilon, y} \in \mathcal{N}_{\varepsilon} .
$$

By construction, $\Phi_{\varepsilon}(y)$ has compact support for any $y \in M$ and it is easy to see that $\Phi_{\varepsilon}$ is a continuous map.

The next result will help us to define a map from $M$ to a suitable sublevel in the Nehari manifold.

Lemma 2.4.2. The function $\Phi_{\varepsilon}$ satisfies

$$
\lim _{\varepsilon \rightarrow 0^{+}} I_{\varepsilon}\left(\Phi_{\varepsilon}(y)\right)=m_{V_{0}}^{\infty} \text {, uniformly in } y \in M \text {. }
$$


Proof. Suppose by contradiction that the lemma is false. Then there exist $\delta_{0}>0,\left\{y_{n}\right\} \subset M$ and $\varepsilon_{n} \rightarrow 0^{+}$such that

$$
\left|I_{\varepsilon_{n}}\left(\Phi_{\varepsilon_{n}}\left(y_{n}\right)\right)-m_{V_{0}}^{\infty}\right| \geq \delta_{0} .
$$

Using Lebesgue's Theorem, we have

$$
\begin{aligned}
\lim _{n \rightarrow \infty}\left\|\Psi_{\varepsilon_{n}, y_{n}}\right\|_{\varepsilon_{n}}^{2} & =\left\|\mathfrak{w}_{V_{0}}\right\|_{H_{V_{0}}^{s}}^{2}, \\
\lim _{n \rightarrow \infty} \int_{\mathbb{R}^{N}} F\left(\Psi_{\varepsilon_{n}, y_{n}}\right) & =\int_{\mathbb{R}^{N}} F\left(\mathfrak{w}_{V_{0}}\right), \\
\lim _{n \rightarrow \infty}\left\|\Psi_{\varepsilon_{n}, y_{n}}\right\|_{H_{V_{0}}^{s}}^{2} & =\left\|\mathfrak{w}_{V_{0}}\right\|_{H_{V_{0}}^{s}}^{2} .
\end{aligned}
$$

This last convergence implies that $\left\{\left\|\Psi_{\varepsilon_{n}, y_{n}}\right\|\right\}$ is bounded. From (2.2.3)

$$
\int_{\mathbb{R}^{N}} \phi_{\varepsilon_{n}, \Psi_{\varepsilon_{n}, y_{n}}} \Psi_{\varepsilon_{n}, y_{n}}^{2} \leq \varepsilon_{n}^{\alpha-\theta} C_{\mathrm{e}}\left\|\Psi_{\varepsilon_{n}, y_{n}}\right\|^{4}
$$

and then

$$
\lim _{n \rightarrow \infty} \int_{\mathbb{R}^{N}} \phi_{\varepsilon_{n}, \Psi_{\varepsilon_{n}, y_{n}}} \Psi_{\varepsilon_{n}, y_{n}}^{2}=0 .
$$

Remembering that $t_{\varepsilon_{n}} \Psi_{\varepsilon_{n}, y} \in \mathcal{N}_{\varepsilon_{n}}$ (see few lines before the Lemma), the condition

$$
I_{\varepsilon_{n}}^{\prime}\left(t_{\varepsilon_{n}} \Psi_{\varepsilon_{n}, y_{n}}\right)\left[t_{\varepsilon_{n}} \Psi_{\varepsilon_{n}, y_{n}}\right]=0
$$

means

$$
\left\|\Psi_{\varepsilon_{n}, y_{n}}\right\|_{\varepsilon_{n}}^{2}+t_{\varepsilon_{n}}^{2} \int_{\mathbb{R}^{N}} \phi_{\varepsilon_{n}, \Psi_{\varepsilon_{n}, y_{n}}} \Psi_{\varepsilon_{n}, y_{n}}^{2}=\int_{\mathbb{R}^{N}} \frac{f\left(t_{\varepsilon_{n}} \Psi_{\varepsilon_{n}, y_{n}}\right)}{t_{\varepsilon_{n}}} \Psi_{\varepsilon_{n}, y_{n}} .
$$

We now prove the following

Claim: $\lim _{n \rightarrow+\infty} t_{\varepsilon_{n}}=1$.

We begin by showing the boundedness of $\left\{t_{\varepsilon_{n}}\right\}$. Since $\varepsilon_{n} \rightarrow 0^{+}$, we can assume $\delta / 2<\delta /\left(2 \varepsilon_{n}\right)$ and then from (2.4.9), using (f5) and making the change of variable $z:=\left(\varepsilon_{n} x-y_{n}\right) / \varepsilon_{n}$, we get

$$
\frac{\left\|\Psi_{\varepsilon_{n}, y_{n}}\right\|_{\varepsilon_{n}}^{2}}{t_{\varepsilon_{n}}^{2}}+\int_{\mathbb{R}^{N}} \phi_{\varepsilon_{n}, \Psi_{\varepsilon_{n}, y_{n}}} \Psi_{\varepsilon_{n}, y_{n}}^{2} \geq \frac{f\left(t_{\varepsilon_{n}} \mathfrak{w}_{V_{0}}(\bar{z})\right)}{\left(t_{\varepsilon_{n}} \mathfrak{w}_{V_{0}}(\bar{z})\right)^{3}} \int_{B_{\delta / 2}} \mathfrak{w}_{V_{0}}^{4}(z),
$$

where $\mathfrak{w}_{V_{0}}(\bar{z}):=\min _{B_{\delta / 2}} \mathfrak{w}_{V_{0}}(z)>0$. If $\left\{t_{\varepsilon_{n}}\right\}$ were unbounded, passing to the limit in $n$ in (2.4.10), the left hand side would tend to 0 (due to $(2.4 .7)$ and (2.4.8)), the right hand side to $+\infty$ (due to (f4)). So we can assume that $t_{\varepsilon_{n}} \rightarrow t_{0} \geq 0$.

Given $\xi>0$, by $(2.2 .1)$, there exists $M_{\xi}>0$ such that

$$
\int_{\mathbb{R}^{N}} \frac{f\left(t_{\varepsilon_{n}} \Psi_{\varepsilon_{n}, y_{n}}\right)}{t_{\varepsilon_{n}}} \Psi_{\varepsilon_{n}, y_{n}} \leq \xi \int_{\mathbb{R}^{N}} \Psi_{\varepsilon_{n}, y_{n}}^{2}+M_{\xi} t_{\varepsilon_{n}}^{q-1} \int_{\mathbb{R}^{N}} \Psi_{\varepsilon_{n}, y_{n}}^{q+1}
$$

Since $\left\{\Psi_{\varepsilon_{n}, y_{n}}\right\}$ is bounded in $H^{s}\left(\mathbb{R}^{N}\right)$, if $t_{0}=0$, from (2.4.11) we deduce

$$
\lim _{n \rightarrow \infty} \int_{\mathbb{R}^{N}} \frac{f\left(t_{\varepsilon_{n}} \Psi_{\varepsilon_{n}, y_{n}}\right)}{t_{\varepsilon_{n}}} \Psi_{\varepsilon_{n}, y_{n}}=0
$$

which joint with (2.4.8) and (2.4.9) led to $\lim _{n \rightarrow \infty}\left\|\Psi_{\varepsilon_{n}, y_{n}}\right\|_{\varepsilon_{n}}^{2}=0$ contradicting (2.4.7). Then $t_{\varepsilon_{n}} \rightarrow t_{0}>0$. Now taking the limit in $n$ in (2.4.9) we arrive at

$$
\left\|\mathfrak{w}_{V_{0}}\right\|_{H_{V_{0}}^{s}}^{2}=\int_{\mathbb{R}^{N}} \frac{f\left(t_{0} \mathfrak{w}_{V_{0}}\right)}{t_{0}} \mathfrak{w}_{V_{0}}
$$


and since $\mathfrak{w}_{V_{0}} \in \mathcal{M}_{V_{0}}$, it has to be $t_{0}=1$, which proves the claim.

Finally, note that

$$
\begin{aligned}
I_{\varepsilon_{n}}\left(\Phi_{\varepsilon_{n}}\left(y_{n}\right)\right) & =\frac{t_{\varepsilon_{n}}^{2}}{2} \int_{\mathbb{R}^{N}}\left|(-\Delta)^{s / 2} \Psi_{\varepsilon_{n}, y_{n}}\right|^{2}+\frac{t_{\varepsilon_{n}}^{2}}{2} \int_{\mathbb{R}^{N}} V\left(\varepsilon_{n} x\right) \Psi_{\varepsilon_{n}, y_{n}}^{2} \\
& +\frac{t_{\varepsilon_{n}}^{4}}{4} \int_{\mathbb{R}^{N}} \phi_{\varepsilon_{n}, \Psi_{\varepsilon_{n}, y_{n}}} \Psi_{\varepsilon_{n}, y_{n}}^{2}-\int_{\mathbb{R}^{N}} F\left(t_{\varepsilon_{n}} \Psi_{\varepsilon_{n}, y_{n}}\right) .
\end{aligned}
$$

and then (by using the claim) $\lim _{n \rightarrow \infty} I_{\varepsilon_{n}}\left(\Phi_{\varepsilon_{n}}\left(y_{n}\right)\right)=E_{V_{0}}\left(\mathfrak{w}_{V_{0}}\right)=m_{V_{0}}^{\infty}$, which contradicts (2.4.6). Thus the Lemma holds.

The remaining part of the chapter mainly follows the arguments of Figueiredo and Siciliano (2016).

By Lemma 2.4.2, $h(\varepsilon):=\left|I_{\varepsilon}\left(\Phi_{\varepsilon}(y)\right)-m_{V_{0}}^{\infty}\right|=o(1)$ for $\varepsilon \rightarrow 0^{+}$uniformly in $y$, and then $I_{\varepsilon}\left(\Phi_{\varepsilon}(y)\right)-m_{V_{0}}^{\infty} \leq h(\varepsilon)$. In particular the sublevel set in the Nehari manifold

$$
\mathcal{N}_{\varepsilon}^{m_{V_{0}}^{\infty}+h(\varepsilon)}:=\left\{u \in \mathcal{N}_{\varepsilon}: I_{\varepsilon}(u) \leq m_{V_{0}}^{\infty}+h(\varepsilon)\right\}
$$

is not empty, since for sufficiently small $\varepsilon$,

$$
\forall y \in M: \Phi_{\varepsilon}(y) \in \mathcal{N}_{\varepsilon}^{m_{V_{0}}^{\infty}+h(\varepsilon)} .
$$

From now on we fix a $\delta>0$ in such a way that $M$ and

$$
M_{2 \delta}:=\left\{x \in \mathbb{R}^{N}: d(x, M) \leq 2 \delta\right\}
$$

are homotopically equivalent ( $d$ denotes the euclidean distance). Take a $\rho=\rho(\delta)>0$ such that $M_{2 \delta} \subset B_{\rho}$ and $\chi: \mathbb{R}^{N} \rightarrow \mathbb{R}^{N}$ be defined as follows

$$
\chi(x)= \begin{cases}x & \text { if }|x| \leq \rho \\ \rho \frac{x}{|x|} & \text { if }|x| \geq \rho .\end{cases}
$$

Define the barycenter map $\beta_{\varepsilon}$

$$
\beta_{\varepsilon}(u):=\frac{\int_{\mathbb{R}^{N}} \chi(\varepsilon x) u^{2}(x)}{\int_{\mathbb{R}^{N}} u^{2}(x)} \in \mathbb{R}^{N}
$$

for all $u \in W_{\varepsilon}$ with compact support.

We will take advantage of the following results (see Figueiredo and Siciliano 2016, Lemma 8 and 9).

Lemma 2.4.3. The function $\beta_{\varepsilon}$ satisfies

$$
\lim _{\varepsilon \rightarrow 0^{+}} \beta_{\varepsilon}\left(\Phi_{\varepsilon}(y)\right)=y, \quad \text { uniformly in } y \in M \text {. }
$$

Lemma 2.4.4. We have

$$
\lim _{\varepsilon \rightarrow 0^{+}} \sup _{\substack{m \in \mathcal{N}_{\varepsilon} \\ V_{0}}} \inf _{y \in M_{\delta}}\left|\beta_{\varepsilon}(u)-y\right|=0 .
$$


In virtue of Lemma 2.4.4, there exists $\varepsilon^{*}>0$ such that

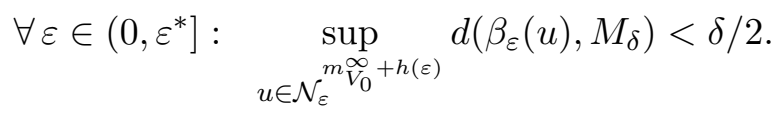

Define now

$$
M^{+}:=\left\{x \in \mathbb{R}^{N}: d(x, M) \leq 3 \delta / 2\right\}
$$

so that $M$ and $M^{+}$are homotopically equivalent.

Now, reducing $\varepsilon^{*}>0$ if necessary, we can assume that Lemma 2.4.3, Lemma 2.4.4 and (2.4.12) hold. Then by standard arguments the composed map

$$
M \stackrel{\Phi_{\varepsilon}}{\longrightarrow} \mathcal{N}_{\varepsilon}^{m_{V_{0}}^{\infty}+h(\varepsilon)} \stackrel{\beta_{\varepsilon}}{\longrightarrow} M^{+} \quad \text { is homotopic to the inclusion map. }
$$

In case $V_{\infty}<\infty$, we eventually reduce $\varepsilon^{*}$ in such a way that also the Palais-Smale condition is satisfied in the interval $\left(m_{V_{0}}^{\infty}, m_{V_{0}}^{\infty}+h(\varepsilon)\right)$, see Proposition 2.3.5. By well known properties of the category, it is

$$
\operatorname{cat}\left(\mathcal{N}_{\varepsilon}^{m_{V_{0}}^{\infty}+h(\varepsilon)}\right) \geq \operatorname{cat}_{M^{+}}(M)
$$

and the Ljusternik-Schnirelmann theory ensures the existence of at least $\operatorname{cat}_{M^{+}}(M)=\operatorname{cat}(M)$ constraint critical points of $I_{\varepsilon}$ on $\mathcal{N}_{\varepsilon}$, hence, by Proposition 2.3.5, solutions of our problem.

If $M$ is not contractible in itself, then the existence of another critical point of $I_{\varepsilon}$ on $\mathcal{N}_{\varepsilon}$ follows from some ideas in Benci et al. (1991). We recall here the main steps for completeness.

The goal is to exhibit a subset $\mathcal{A} \subset \mathcal{N}_{\varepsilon}$ such that

i) $\mathcal{A}$ is not contractible in $\mathcal{N}_{\varepsilon}^{m_{V_{0}}^{\infty}+h(\varepsilon)}$,

ii) $\mathcal{A}$ is contractible in $\mathcal{N}_{\varepsilon}^{\bar{c}}=\left\{u \in \mathcal{N}_{\varepsilon}: I_{\varepsilon}(u) \leq \bar{c}\right\}$, for some $\bar{c}>m_{V_{0}}^{\infty}+h(\varepsilon)$.

This would imply, since the Palais-Smale holds, that there is a critical level between $m_{V_{0}}^{\infty}+h(\varepsilon)$ and $\bar{c}$.

First note that when $M$ is not contractible and bounded the compact set $\mathcal{A}:=\Phi_{\varepsilon}(M)$ can not be contractible in $\mathcal{N}_{\varepsilon}^{m_{V_{0}}^{\infty}+h(\varepsilon)}$, proving i).

Let us denote, for $u \in W_{\varepsilon} \backslash\{0\}$, with $t_{\varepsilon}(u)>0$ the unique positive number such that $t_{\varepsilon}(u) u \in \mathcal{N}_{\varepsilon}$. Choose a function $u^{*} \in W_{\varepsilon}$ be such that $u^{*} \geq 0, I_{\varepsilon}\left(t_{\varepsilon}\left(u^{*}\right) u^{*}\right)>m_{V_{0}}^{\infty}+h(\varepsilon)$ and consider the compact and contractible cone

$$
\mathfrak{C}:=\left\{t u^{*}+(1-t) u: t \in[0,1], u \in \mathcal{A}\right\}
$$

Observe that, since the functions in $\mathfrak{C}$ have to be positive on a set of nonzero measure, it is $0 \notin \mathfrak{C}$. Now we project this cone on $\mathcal{N}_{\varepsilon}$ : let

$$
t_{\varepsilon}(\mathfrak{C}):=\left\{t_{\varepsilon}(w) w: w \in \mathfrak{C}\right\} \subset \mathcal{N}_{\varepsilon}
$$

and set

$$
\bar{c}:=\max _{t_{\varepsilon}(\mathfrak{C})} I_{\varepsilon}>m_{V_{0}}^{\infty}+h(\varepsilon)
$$

(indeed the maximum is achieved being $t_{\varepsilon}(\mathfrak{C})$ compact). Of course $\mathcal{A} \subset t_{\varepsilon}(\mathfrak{C}) \subset \mathcal{N}_{\varepsilon}$ and $t_{\varepsilon}(\mathfrak{C})$ is contractible in $\mathcal{N}_{\varepsilon}^{\bar{c}}$ : we deduce ii).

Then there is a critical level for $I_{\varepsilon}$ greater than $m_{V_{0}}^{\infty}+h(\varepsilon)$, hence different from the previous ones we have found.

The fact that the solutions we have found are positive, follows from the fact that all the above 
procedure can be applied to the functional

$$
I_{\varepsilon}^{+}:=\frac{1}{2} \int_{\mathbb{R}^{N}}\left|(-\Delta)^{s / 2} u\right|^{2}+\frac{1}{2} \int_{\mathbb{R}^{N}} V(\varepsilon x) u^{2}+\frac{1}{4} \int_{\mathbb{R}^{N}} \phi_{\varepsilon, u} u^{2}-\int_{\mathbb{R}^{N}} F\left(u^{+}\right),
$$

with $u^{+}=\max \{u, 0\}$. The proof of Theorem 2.1.1 is complete. 


\section{Chapter 3}

\section{Positive solutions to the Schrödinger-Poisson system under asymptotically cubic nonlinearity}

\subsection{Introduction}

Elliptic systems involving the Schrödinger and the Maxwell equations have attracted a lot of interest in mathematical physics in the last decades, indeed they appear in many physical situations, especially in nonrelativistic Quantum Mechanics. Many authors have studied this intriguing problem, which takes into account the interaction of a nonrelativistic particle with its own electromagnetic field, that it is almost impossible to give a complete list of references on the topic. The huge literature existing deals mainly with the equations settled in the whole space $\mathbb{R}^{N}$, while only few works study the case of bounded domain where boundary condition may even prevent the existence of solutions, and different type of solutions have been found (radial, nonradial, sign-changing, high-energy, etc.).

We cite here the pioneering paper of Benci and Fortunato (Benci and Fortunato, 1998) since they first gave a deduction of the equations which describe the interaction of the matter field with the electromagnetic field in the framework of Abelian Gauge Theories, in place of the usual and classical Hartree and Thomas-Fermi-von Weizsäcker Theory of atoms and molecules (see e.g. Benguria et al. 1981; Catto and Lions 1992).

Without entering in details in the physical and mathematical derivation of the equations, the search of stationary solutions

$$
\psi(x, t)=u(x) e^{-i \omega t} \in \mathbb{C}, \quad u(x), \omega \in \mathbb{R},
$$

of the so called Schrödinger-Maxwell system in the purely electrostatic case, i.e.

$$
\phi(x, t)=\phi(x), \quad \mathbf{A}(x, t)=\mathbf{0},
$$

(where $(\phi, \mathbf{A})$ is the gauge potential of the electromagnetic field) leads to the following model problem, known as Schrödinger-Poisson system:

$$
\begin{cases}-\Delta u+\omega u+\phi u=|u|^{p-2} u & \text { in } \Omega \\ -\Delta \phi=u^{2} & \text { in } \Omega\end{cases}
$$

in the unknowns $u$ and $\phi$. Here the nonlinearity describes the interaction between many particles. We recall that the physically interesting case is $p=8 / 3$ but other values of $p$ are admissible from a mathematical point of view, and even more general nonlinearities which are not of power type are admissible.

Since we are not interested in the value of $\omega$, we will set $\omega=1$ in the following; everything also 
work for $\omega>0$.

In this chapter we are interested in the existence of positive solutions for Schrödinger-Poisson like systems under an asymptotically cubic nonlinearity, which has never been considered before. In this way the terms $\phi u$ and the nonlinearity are "comparable" at infinity, in the sense that $\phi u$ is 3-homogeneous hence "behaves like the nonlinearity" as it will be specified later (see Section 3.2) so that in our case the terms $f(u)$ and $\phi u$ in the equation will be in competition and thus the geometry of the energy functional becomes delicate.

The Schrödinger-Poisson system addressed here is of type

$$
\begin{cases}-\Delta u+u+\lambda \phi u=f(u) & \text { in } \mathbb{R}^{3} \\ -\Delta \phi=u^{2} & \text { in } \mathbb{R}^{3} .\end{cases}
$$

We have decided to put a suitable parameter $\lambda>0$ since it has a role in some bifurcation results, as well as some existence or nonexistence results depends on this parameter joint with some assumptions on the nonlinearity $f$, see e.g. Ruiz (2006); Ruiz and Siciliano (2008), and this will be exactly our case.

More specifically we are interested in a Schrödinger-Poisson problem on suitable domains $\Omega$ of type

$$
\begin{cases}-\Delta u+u+\lambda \phi u=f(u) & \text { in } \Omega \\ -\Delta \phi=\widetilde{u}^{2} & \text { in } \mathbb{R}^{3} \\ u=0 & \text { on } \partial \Omega\end{cases}
$$

where, hereafter, given a function $v \in H_{0}^{1}(\Omega)$ we denote with $\widetilde{v}$ its trivial extension to the whole $\mathbb{R}^{3}$, i.e.

$$
\widetilde{v}(x)=v(x) \text { if } x \in \Omega, \quad \widetilde{v}(x)=0 \quad \text { if } x \in \mathbb{R}^{3} \backslash \Omega \text {. }
$$

Evidently $\widetilde{v} \in H^{1}\left(\mathbb{R}^{3}\right)$.

It is worth to point out that problems like (3.1.2) have already appeared in the literature. For example in Alves et al. (2017, Section 3) it is considered the case $\Omega=B_{R}$, where the authors are interested in sign changing solutions, under the "super cubic" condition $\lim _{t \rightarrow \infty} F(t) / t^{4}=+\infty$. Similar problems are considered in Ianni (2013) where it is proved the existence of sign changing solutions which change sign an arbitrary number of times.

Our assumptions on $f$ are the following

(f1) $f \in C(\mathbb{R}, \mathbb{R})$;

(f2) $f(t)=0$ for $t \leq 0$;

(f3) $\lim _{t \rightarrow 0} f(t) / t=0$;

(f4) there is $s>0$ such that $\lim _{t \rightarrow \infty} f(t) / t^{3}=1 / s$ and $f(t) / t^{3}<1 / s$ for all $t>0$;

(f5) the function $t \mapsto f(t) / t^{3}$ is strictly increasing on $(0,+\infty)$;

(f6) setting $F(t):=\int_{0}^{t} f(\tau) d \tau$, it holds

$$
\lim _{t \rightarrow \infty}[f(t) t-4 F(t)]=\infty .
$$

Given $s>0$, a concrete nonlinearity satisfying (f1)-(f6) is the function defined by

$$
f(t):= \begin{cases}\frac{t^{5}}{1+s t^{2}} & \text { if } t \geq 0 \\ 0 & \text { if } t \leq 0 .\end{cases}
$$


Actually we will consider the case $s=1$, without any loss of generality. Indeed it is easily seen that, if $f$ satisfies (f1)-(f6) also the function $t \mapsto s f(t)$ does.

We will prove the following results

Theorem 3.1.1. Under our assumptions on $f$, for any $\lambda, R$ small, problem (3.1.2) with $\Omega=\mathbb{R}^{3} \backslash B_{R}$ has a positive solution in $H_{0, \text { rad }}^{1}\left(\mathbb{R}^{3} \backslash B_{R}\right)$.

Theorem 3.1.2. Under our assumptions on $f$, for any $\lambda, R>0$, problem (3.1.2) with $\Omega=B_{R}$ has a positive radial solution in $H_{0, r a d}^{1}\left(B_{R}\right)$.

Of course the radial symmetry in Theorem 3.1.2 is not really used in the proof, hence a similar existence result holds for a (possible nonradial) domain. We decided to state the Theorem in the radial case in view of possible future applications to least energy nodal solutions to the SchrödingerPoisson system with an asymptotically cubic nonlinearity, in the same spirit of Maia et al. (2015).

Our approach is variational. As we have seen in Chapter 1, the solutions are critical points of the following functional

$$
I_{\Omega}(u)=\frac{1}{2} \int_{\Omega}|\nabla u|^{2} d x+\frac{1}{2} \int_{\Omega} u^{2} d x+\frac{\lambda}{4} \int_{\Omega} \phi_{\widetilde{u}} u^{2} d x-\int_{\Omega} F(u) d x, \quad u \in H_{0}^{1}(\Omega)
$$

with $\Omega=\mathbb{R}^{3} \backslash B_{R}$ or $\Omega=B_{R}$ (in this chapter $B_{R}$ denotes the ball in $\mathbb{R}^{3}$ with center at the origin and radius $R$ ), where $\phi_{\widetilde{u}}$ is the unique solution of the second equation of the system (see Chapter 1). The main difficulty is exactly due to the fact that we are dealing with an asymptotically cubic nonlinearity, in particular the Ambrosetti-Rabinowitz condition is not available.

Now some remarks on the assumptions are in order.

(1) First of all, condition (f2) is very natural since we will be interested in positive solutions: it will guarantee that any nontrivial solution has to be positive. Indeed if $v$ is a nontrivial solution and $v^{-}$denotes its negative part, by multiplying the first equation of (3.1.1) by $v^{-}$and hence integrating on $\Omega$ we get

$$
\int_{\Omega}\left|\nabla\left(v^{-}\right)\right|^{2} d x+\int_{\Omega}\left(v^{-}\right)^{2} d x+\lambda \int_{\Omega} \phi_{\widetilde{v}}\left(v^{-}\right)^{2} d x=0
$$

and then $v \geq 0$. Then

$$
\Delta(-v)-(-v)-\lambda \phi_{\widetilde{v}}(-v) \geq 0 .
$$

Now we recall a maximum principle (see e.g. Protter and Weinberger 1999, Theorem 6, pg. 64), whose statement here is conveniently adapted to our situation.

Theorem 3.1.3 (Maximum principle). Let $u$ satisfy

$$
(\Delta+h)(u) \geq 0
$$

with $h \leq 0$ and bounded. If $u$ attains a nonnegative maximum $M$ at an interior point of $\Omega$, then $u=M$.

Defining $h:=-1-\lambda \phi_{\widetilde{v}}$, it is clear that $h<0$ and $|h| \leq 1+\lambda \phi_{\widetilde{v}}(0)$. If it were $v(\bar{x})=0$ for some point $\bar{x} \in \Omega$ we would have $v=0$ in $\Omega$, by the maximum principle, in contradiction with the fact that $v$ is not identically zero. Therefore $v>0$ in $\Omega$.

(2) Secondly, the name "asymptotically cubic" is given due to condition (f4). Observe that under this condition there is a "competition" between the terms $\phi u$ and the nonlinearity in the equation since $\phi u$ is, roughly speaking of order 3. Moreover $f$ cannot satisfy the so called AmbrosettiRabinowitz condition (for the Schrödinger-Poisson system), i.e.

$$
\exists \theta>4: 0<\theta F(t) \leq t f(t),
$$

since this last one gives $f(t) \geq C t^{\theta-1}$ for $t$ large. 
In particular Theorem 3.1.2 complete the study made in Pisani and Siciliano (2008) where a nonlinerity satisfying the Ambrosetti-Rabinowitz condition was considered.

(3) From (f5) one deduce that $t \mapsto f(t) t-4 F(t)$ is strictly increasing on $(0, \infty)$ and then, since in zero is zero, it has to be $f(t) t-4 F(t)>0$ for every $t>0$. The short proof is given in Liu (2010, Lemma 2.3) but we write it here for completeness.

Let us define $H(t):=t f(t)-4 F(t)$ and $0<s<t$. Then

$$
\begin{aligned}
H(t)-H(s) & =4\left[\frac{1}{4}(f(t) t-f(s) s)-(F(t)-F(s))\right] \\
& =4\left[\int_{0}^{t} \frac{f(t)}{t^{3}} \tau^{3} d \tau-\int_{0}^{s} \frac{f(s)}{s^{3}} \tau^{3} d \tau-\int_{s}^{t} \frac{f(\tau)}{\tau^{3}} \tau^{3} d \tau\right] \\
& =4\left[\int_{s}^{t}\left(\frac{f(t)}{t^{3}}-\frac{f(\tau)}{\tau^{3}}\right) \tau^{3} d \tau+\int_{0}^{s}\left(\frac{f(t)}{t^{3}}-\frac{f(s)}{s^{3}}\right) \tau^{3} d \tau\right]>0
\end{aligned}
$$

so that $H(s)<H(t)$.

Moreover hypothesis (f5) is useful whenever one want to use the method of the Nehari manifold. More specifically, any $u \neq 0$ can be projected at most once on the so called Nehari manifold associated to the problem, i.e.

$$
\mathcal{N}:=\left\{u \in H_{0}^{1}(\Omega) \backslash\{0\}: I_{\Omega}^{\prime}(u)[u]=0 \Leftrightarrow \int_{\Omega}|\nabla u|^{2} d x+\int_{\Omega} u^{2} d x+\lambda \int_{\Omega} \phi_{\widetilde{u}} u^{2} d x=\int_{\Omega} f(u) u d x\right\} .
$$

Indeed, for $u \neq 0$ let us define for convenience, $\gamma_{u}(t):=I_{\Omega}(t u)$; then

$$
\gamma_{u}^{\prime}(1):=\left.\frac{d}{d t} I_{\Omega}(t u)\right|_{t=1}=I_{\Omega}^{\prime}(u)[u]
$$

Now let $u \in \mathcal{N}$ : for $t \in(0,1)$ by (f5) we have

$$
t \gamma_{u}^{\prime}(t)=I_{\Omega}^{\prime}(t u)[t u]>t^{4}\left(\|u\|^{2}+\lambda \int_{\Omega} \phi_{\widetilde{u}} u^{2} d x-\int_{\Omega} \frac{f(t u)}{t^{3}} u d x\right)>t^{4} \gamma_{u}^{\prime}(1)=0
$$

for $t>1$ we have

$$
t \gamma_{u}^{\prime}(t)=I_{\Omega}^{\prime}(t u)[t u]<t^{4}\left(\|u\|^{2}+\lambda \int_{\Omega} \phi_{\widetilde{u}} u^{2} d x-\int_{\Omega} \frac{f(t u)}{t^{3}} u d x\right)<t^{4} \gamma_{u}^{\prime}(1)=0 .
$$

Observe also that if $u \in \mathcal{N}$ then the fibering maps $\gamma_{u}$ achieves the maximum value on $t=1$, i.e.

$$
I_{\Omega}(t u)<I_{\Omega}(u) \text { for every } t \geq 0, t \neq 1 .
$$

To this aim define the function

$$
\xi(t):=\left(\frac{t^{2}}{2}-\frac{t^{4}}{4}\right)\left[|\nabla u|^{2}+u^{2}\right]+\frac{t^{4}}{4} f(u) u-F(t u), \quad t \geq 0 .
$$

In the set $\left\{x \in \mathbb{R}^{3}: u(x) \neq 0\right\}$ which has positive measure, for any $t>0$

$$
\xi^{\prime}(t)=t\left(1-t^{2}\right)\left[|\nabla u|^{2}+u^{2}\right]+t^{3} u^{4}\left[\frac{f(u)}{u^{3}}-\frac{f(t u)}{t^{3} u^{3}}\right],
$$

and thus, by (f5), $\xi(t)<\xi(1)$, for every $t \geq 0, t \neq 1$. Then, after integration on $\Omega$ and using that 
$\gamma_{u}^{\prime}(1)=0$, we have

$$
\begin{aligned}
I_{\Omega}(t u) & =\left(\frac{t^{2}}{2}-\frac{t^{4}}{4}\right) \int_{\Omega}\left[|\nabla u|^{2}+u^{2}\right] d x+\int_{\Omega}\left[\frac{t^{4}}{4} f(u) u-F(t u)\right] d x \\
& <\frac{1}{4} \int_{\Omega}\left[|\nabla u|^{2}+u^{2}\right] d x+\int_{\Omega}\left[\frac{1}{4} f(u) u-F(u)\right] d x \\
& =I_{\Omega}(u) .
\end{aligned}
$$

(4) Finally note that in particular from (f1), (f3) and (f4), given $\varepsilon>0$ and $q \in(4,6)$, there exists a constant $M_{\varepsilon}>0$ such that

$$
|f(t)| \leq \varepsilon|t|+M_{\varepsilon}|t|^{q-1} \quad \text { and } \quad|F(t)| \leq \frac{\varepsilon}{2} t^{2}+\frac{M_{\varepsilon}}{q}|t|^{q}, \quad \forall t \in \mathbb{R}
$$

\subsection{Few preliminaries}

As it is standard by now, when dealing with Schrödinger-Poisson systems a useful approach consists in rewriting problem (3.1.1) as a single equation involving a nonlocal term. For a detailed explanation about this reduction argument, see Section 1.4 in Chapter 1. We remark here the facts that we need. Indeed for every fixed $u \in H^{1}\left(\mathbb{R}^{3}\right)$, by the Riesz representation theorem, there is a unique solution $\phi_{u} \in D^{1,2}\left(\mathbb{R}^{3}\right)$ to the second equation in (3.1.1). Moreover

(a1) for every $x \in \mathbb{R}^{3}, \phi_{u}(x)$ has the integral representation

$$
\phi_{u}(x)=\int_{\mathbb{R}^{3}} \frac{u^{2}(y)}{|x-y|} d y .
$$

In particular under the dilation $u \mapsto t u, \phi_{u}$ changes into $t^{2} \phi_{u}$. In this sense one says that the term $\phi u$ appearing in the equation is homogeneous of degree 3 . Clearly from this representation, $\phi_{u}>0$ and if $u$ is radial then $\phi_{u}$ is also radial and we have (with some abuse of notation) the Newton formula

$$
\phi_{u}(r)=\frac{1}{r} \int_{0}^{\infty} u^{2}(s) s \min \{s, r\} d s
$$

(a2) there are constant $C_{0}, C_{1}>0$ such that

$$
\left\|\phi_{u}\right\|_{D} \leq C_{0}\|u\|^{2}, \quad \int_{\mathbb{R}^{3}} \phi_{u} u^{2} d x \leq C_{1}\|u\|^{4} .
$$

(a3) if $u_{n} \rightarrow u$ in $H_{r a d}^{1}\left(\mathbb{R}^{3}\right)$ then

$$
\phi_{u_{n}} \rightarrow \phi_{u} \text { in } D^{1,2}\left(\mathbb{R}^{3}\right) \quad \text { and } \quad \int_{\mathbb{R}^{3}} \phi_{u_{n}} u_{n}^{2} \rightarrow \int_{\mathbb{R}^{3}} \phi_{u} u^{2} .
$$

For these properties also you can see e.g. Ruiz (2006).

A similar reduction procedure can be made in a domain $\Omega$ for the problem (3.1.2), and then we are reduce to solve the equation

$$
-\Delta u+u+\lambda \phi_{\widetilde{u}} u=f(u), \quad u \in H_{0}^{1}(\Omega) .
$$

Throughout this chapter, given a function $u \in H_{0}^{1}(\Omega), \widetilde{u}$ will denote the trivial extension of $u$ to the whole space $\mathbb{R}^{3}$, i.e., $\widetilde{u}(x)=u(x)$ for $x \in \Omega$ and $\widetilde{u}(x)=0$ for $x$ outside $\Omega$. In particular the 
identity

$$
\left.\phi_{\widetilde{u}}\right|_{\Omega}=\int_{\Omega} \frac{u^{2}(y)}{|\cdot-y|} d y
$$

holds.

We begin by recalling a result on the whole $\mathbb{R}^{3}$. This will have a major role in all our analysis. In the paper Azzollini et al. (2010) it was studied the problem

$$
\begin{cases}-\Delta u+\lambda \phi u=g(u) & \text { in } \mathbb{R}^{3} \\ -\Delta \phi=\lambda u^{2} & \text { in } \mathbb{R}^{3}\end{cases}
$$

under the following assumptions on $g$ :

(g1) $g \in C(\mathbb{R}, \mathbb{R})$,

(g2) $-\infty<\liminf \operatorname{lo}_{t \rightarrow 0^{+}} g(t) / t \leq \lim \sup _{t \rightarrow 0^{+}} g(t) / t=-m<0$,

(g3) $-\infty \leq \lim \sup _{t \rightarrow \infty} g(t) / t^{5} \leq 0$,

(g4) there exists $t_{0}>0$ such that $G\left(t_{0}\right):=\int_{0}^{t_{0}} g(t) d t>0$.

In other words $g$ is a general nonlinearity satisfying the Berestycki-Lions assumptions. The authors prove that there is a $\lambda_{0}>0$ such that for every $\lambda \in\left(0, \lambda_{0}\right)$ the problem (3.2.1) has a nontrivial positive and radial solution.

Our problem (3.1.1) can be written as (3.2.1) just renaming the nonlinearity and the potential $\phi$; then it is easily seen that we have the following

Lemma 3.2.1. Under condition (f1)-(f6) there is a $\lambda_{0}>0$ such that for every $\lambda \in\left(0, \lambda_{0}\right)$ problem (3.1.1) has a positive and radial solution $\mathfrak{u}$.

The smallness of $\lambda$ is a necessary condition in order to have a solution for problem (3.1.1). In our case, that is in presence of a nonlinearity which is not of power type, this can be seen in the following way. Let $u_{0} \in H^{1}\left(\mathbb{R}^{3}\right)$ be a positive solution of (3.1.1) with $\phi_{0}:=\phi_{u_{0}} \in D^{1,2}\left(\mathbb{R}^{3}\right)$. Multiplying the first equation of (3.1.1) by $u_{0}$ and integrating on $\mathbb{R}^{3}$ we obtain

$$
0<\left\|u_{0}\right\|^{2}=\int_{\mathbb{R}^{3}}\left(\frac{f\left(u_{0}\right)}{u_{0}^{3}}-\lambda \frac{\phi_{0}}{u_{0}^{2}}\right) u_{0}^{4} d x<\int_{\mathbb{R}^{3}}\left(1-\lambda \frac{\phi_{0}}{u_{0}^{2}}\right) u_{0}^{4} d x=\int_{\mathbb{R}^{3}}\left(u_{0}^{4}-\lambda \phi_{0} u_{0}^{2}\right) d x
$$

so that

$$
\lambda<\frac{\int_{\mathbb{R}^{3}} u_{0}^{4} d x}{\int_{\mathbb{R}^{3}} \phi_{0} u_{0}^{2} d x} .
$$

We define the set made by scalar multiple of solutions, precisely

$$
\mathcal{S}:=\left\{\left\|\phi_{u}\right\|_{D}^{-1 / 2} u \in H^{1}\left(\mathbb{R}^{3}\right) \backslash\{0\}: u \text { solves }(3.1 .1)\right\} \neq \emptyset .
$$

Now if $v \in \mathcal{S}$ then $v=\left\|\phi_{u}\right\|_{D}^{-1 / 2} u$ for some solution $u$ and $\left\|\phi_{v}\right\|_{D}=1$. Using (a2) we have

$$
1=\left\|\phi_{v}\right\|_{D}^{2}=\int_{\mathbb{R}^{3}}\left|\nabla \phi_{v}\right|^{2} d x=\int_{\mathbb{R}^{3}} \phi_{v} v^{2} d x \leq C_{1}\|v\|^{4}
$$


then $K_{1}:=\inf _{v \in \mathcal{S}}\|v\|^{4}>0$. Moreovar recalling (3.2.3),

$$
\lambda<\frac{\left\|\phi_{u}\right\|_{D}^{-2} \int_{\mathbb{R}^{3}} u^{4} d x}{\left\|\phi_{u}\right\|_{D}^{-2} \int_{\mathbb{R}^{3}} \phi_{u} u^{2} d x}=\frac{\int_{\mathbb{R}^{3}} v^{4} d x}{\int_{\mathbb{R}^{3}} \phi_{v} v^{2} d x}=\int_{\mathbb{R}^{3}} v^{4} d x \leq C_{\text {opt }}\|v\|^{4}
$$

where $C_{\text {opt }}>0$ is the optimal constant of the embedding $H^{1}\left(\mathbb{R}^{3}\right) \hookrightarrow L^{4}\left(\mathbb{R}^{3}\right)$. But then $\lambda / C_{\text {opt }} \leq K_{1}$ i.e. $\lambda \leq K_{1} C_{\text {opt }}$.

If $\mathfrak{u}$ is the solution given in Lemma 3.2.1, setting with abuse of notation $\mathfrak{u}(x)=\mathfrak{u}(r), \phi_{\mathfrak{u}}(x)=$ $\phi_{\mathfrak{u}}(r)$ with $r=|x|$, recalling (3.2.2) we have also

$$
\operatorname{meas}\left\{r>0: \mathfrak{u}^{2}(r)-\lambda \phi_{\mathfrak{u}}(r)>0\right\}>0
$$

and

$$
\mathcal{R}:=\sup \left\{r>0: \mathfrak{u}^{2}(r)-\lambda \phi_{\mathfrak{u}}(r)>0\right\} \in(0,+\infty]
$$

\subsection{A positive radial solution in the complementary of a ball}

We decide to consider the problem in the complementary of a ball since we think it may be useful in other context.

For every $R>0$ let us consider the following problem

$$
\begin{cases}-\Delta u+u+\lambda \phi u=f(u) & \text { in } \mathbb{R}^{3} \backslash B_{R}, \\ -\Delta \phi=\widetilde{u}^{2} & \text { in } \mathbb{R}^{3}, \\ u=0 & \text { on } \partial\left(\mathbb{R}^{3} \backslash B_{R}\right)\end{cases}
$$

under our assumptions on $f$.

Let us observe that a similar system has been considered in Alves et al. (2017, Section 3) however with different hypotheses on $f$.

Let us define the $C^{1}$ functional $I_{\mathbb{R}^{3} \backslash B_{R}}: H_{0, \text { rad }}^{1}\left(\mathbb{R}^{3} \backslash B_{R}\right) \rightarrow \mathbb{R}$,

$$
I_{\mathbb{R}^{3} \backslash B_{R}}(u):=\frac{1}{2} \int_{\mathbb{R}^{3} \backslash B_{R}}\left(|\nabla u|^{2}+u^{2}\right) d x+\frac{\lambda}{4} \int_{\mathbb{R}^{3} \backslash B_{R}} \phi_{\widetilde{u}} u^{2} d x-\int_{\mathbb{R}^{3} \backslash B_{R}} F(u) d x,
$$

whose critical points are easily seen to be weak solutions of (3.3.1). Then we are reduced to prove it has a critical point.

Theorem 3.1.1 will be proved by using the Mountain Pass Theorem, see Ambrosetti and Rabinowitz (1973).

\subsubsection{Mountain Pass geometry of $I_{\mathbb{R}^{3} \backslash B_{R}}$}

Let $\mathfrak{u}$ be the positive radial solution given in Lemma 3.2.1 and $\mathcal{R}$ be defined in (3.2.4). Our first purpose is to prove that $I_{\mathbb{R}^{3} \backslash B_{R}}$ has the Mountain Pass geometry.

Lemma 3.3.1. For any $0<R<\mathcal{R}$, assume that (f1),(f3) and (f4) are satisfied. Then, there exist positive numbers $b, \rho$ and $e \in H_{0, \text { rad }}^{1}\left(\mathbb{R}^{3} \backslash B_{R}\right)$ such that

(i) $I_{\mathbb{R}^{3} \backslash B_{R}}(u) \geq b$, for every $u \in H_{0, \text { rad }}^{1}\left(\mathbb{R}^{3} \backslash B_{R}\right)$ such that $\|u\|_{\mathbb{R}^{3} \backslash B_{R}}=\rho$;

(ii) $\|e\|_{\mathbb{R}^{3} \backslash B_{R}}>\rho$ and $I_{\mathbb{R}^{3} \backslash B_{R}}(e)<0$. 
Proof. To prove (i), fixed $\varepsilon \in(0,1)$, for any $u \in H_{0, \text { rad }}^{1}\left(\mathbb{R}^{3} \backslash B_{R}\right)$ we have

$$
\begin{aligned}
I_{\mathbb{R}^{3} \backslash B_{R}}(u) & =\frac{1}{2}\|u\|_{\mathbb{R}^{3} \backslash B_{R}}^{2}+\frac{\lambda}{4} \int_{\mathbb{R}^{3} \backslash B_{R}} \phi_{\widetilde{u}} u^{2} d x-\int_{\mathbb{R}^{3} \backslash B_{R}} F(u) d x \\
& \geq \frac{1}{2}\|u\|_{\mathbb{R}^{3} \backslash B_{R}}^{2}-\int_{\mathbb{R}^{3} \backslash B_{R}}\left(\frac{\varepsilon}{2} u^{2}+\frac{M_{\varepsilon}}{q}|u|^{q}\right) d x \\
& \geq \frac{1-\varepsilon}{2}\|u\|_{\mathbb{R}^{3} \backslash B_{R}}^{2}-\frac{M_{\varepsilon}}{q} \int_{\mathbb{R}^{3} \backslash B_{R}}|u|^{q} d x \\
& \geq \frac{1-\varepsilon}{2}\|u\|_{\mathbb{R}^{3} \backslash B_{R}}^{2}-M_{\varepsilon}\|u\|_{\mathbb{R}^{3} \backslash B_{R}}^{q},
\end{aligned}
$$

by the Sobolev embedding $H_{0, \text { rad }}^{1}\left(\mathbb{R}^{3} \backslash B_{R}\right) \hookrightarrow L^{q}\left(\mathbb{R}^{3} \backslash B_{R}\right)$. Taking $\rho:=\|u\|_{\mathbb{R}^{3} \backslash B_{R}}$ sufficiently small such that

$$
I_{\mathbb{R}^{3} \backslash B_{R}}(u)=b:=\frac{1-\varepsilon}{2} \rho^{2}-M_{\varepsilon} \rho^{q}>0
$$

we conclude the proof.

Let us pass to show (ii). We follows some ideas of Stuart and Zhou (1999). The function $e$ will be constructed starting from the solution $\mathfrak{u}$ (see Lemma 3.2.1). Since $0<R<\mathcal{R}$, there are $r_{1}, r_{4}$ real numbers with $0<R<r_{1}<r_{4}<\mathcal{R}$ such that

$$
\mathfrak{u}^{2}(r)-\lambda \phi_{\mathfrak{u}}(r)>0, \text { for } r_{1} \leq r \leq r_{4} .
$$

Given $0<r<\rho$, we use the notation $A_{r, \rho}$ for the annulus $B_{\rho} \backslash \bar{B}_{r}$. Let $\delta>0$ be such that $\int_{A_{r_{1}, r_{4}}}\left(\mathfrak{u}^{2}-\lambda \phi_{\mathfrak{u}}\right) \mathfrak{u}^{2} d x>3 / 2 \delta$. We can find numbers $r_{2}, r_{3}>0$ that depend on $R$ such that $r_{1}<r_{2}<r_{3}<r_{4}$ and

$$
\int_{A_{r_{2}, r_{3}}}\left(\mathfrak{u}^{2}-\lambda \phi_{\mathfrak{u}}\right) \mathfrak{u}^{2} d x>\delta, \quad \text { and }, \quad \int_{A_{r_{i}, r_{i+1}}}\left(\mathfrak{u}^{2}+\lambda \phi_{\mathfrak{u}}\right) \mathfrak{u}^{2} d x<\frac{\delta}{4}, \text { for } i=1,3
$$

Let $\nu, \eta \in C^{\infty}\left(\mathbb{R}^{3}\right)$ be radial cut-off functions with

- $0 \leq \nu \leq 1, \nu=0$ in $B_{r_{1}}, \nu$ strictly increasing in $A_{r_{1}, r_{2}}, \nu=1$ outside $B_{r_{2}}$,

- $0 \leq \eta \leq 1, \eta=1$ in $B_{r_{3}}, \eta$ strictly decreasing in $A_{r_{3}, r_{4}}, \eta=0$ outside $B_{r_{4}}$.

Define $\widehat{e}_{t}:=t \nu \eta \mathfrak{u}, t>0$. Then $\widehat{e}_{t} \in H^{1}\left(\mathbb{R}^{3}\right)$, and taking $e_{t}(x):=\widehat{e}_{t}(x)$ for $x \in \mathbb{R}^{3} \backslash B_{R}$, it is clear that $e_{t} \in H_{0}^{1}\left(\mathbb{R}^{3} \backslash B_{R}\right), \widehat{e}_{t}=\widetilde{e}_{t}$ and $\lim _{t \rightarrow \infty}\left\|e_{t}\right\|_{\mathbb{R}^{3} \backslash B_{R}}=+\infty$. Then

$$
\begin{aligned}
I_{\mathbb{R}^{3} \backslash B_{R}}\left(e_{t}\right) & =\frac{1}{2}\left\|e_{t}\right\|_{\mathbb{R}^{3} \backslash B_{R}}^{2}+\frac{\lambda}{4} \int_{\mathbb{R}^{3} \backslash B_{R}} \phi_{\widetilde{e_{t}}} e_{t}^{2} d x-\int_{\mathbb{R}^{3} \backslash B_{R}} F\left(e_{t}\right) d x \\
& =\frac{1}{2}\left\|\widehat{e}_{t}\right\|^{2}+\frac{\lambda}{4} \int_{\mathbb{R}^{3} \backslash B_{R}} \phi_{\widehat{e}_{t}} \widehat{e}_{t}^{2} d x-\int_{\mathbb{R}^{3} \backslash B_{R}} F\left(\widehat{e}_{t}\right) d x \\
& =\frac{t^{2}}{2}\|\nu \mathfrak{u} \eta\|^{2}+\lambda \frac{t^{4}}{4} \int_{\mathbb{R}^{3} \backslash B_{R}} \phi_{\nu \mathfrak{u} \eta}(\nu \mathfrak{u} \eta)^{2} d x-\int_{\mathbb{R}^{3} \backslash B_{R}} F(t \nu \mathfrak{u} \eta) d x \\
& <\frac{t^{4}}{4}\left[\frac{2}{t^{2}}\|\mathfrak{u}\|^{2}+\lambda \int_{A_{r_{1}, r_{4}}} \phi_{\nu \mathfrak{u} \eta}(\nu \mathfrak{u} \eta)^{2} d x-\int_{A_{r_{1}, r_{4}}} \frac{F(t \nu \mathfrak{u} \eta)}{(t \nu \mathfrak{u} \eta)^{4} / 4}(\nu \mathfrak{u} \eta)^{4} d x\right]
\end{aligned}
$$


from which

$$
\begin{aligned}
\limsup _{t \rightarrow \infty} \frac{I_{\mathbb{R}^{3} \backslash B_{R}}\left(e_{t}\right)}{t^{4} / 4} & \leq \lambda \int_{A_{r_{1}, r_{4}}} \phi_{\nu \mathfrak{u} \eta}(\nu \mathfrak{u} \eta)^{2} d x+\limsup _{t \rightarrow \infty}\left[\frac{2}{t^{2}}\|\mathfrak{u}\|^{2}-\int_{A_{r_{1}, r_{4}}} \frac{F(t \nu \mathfrak{u} \eta)}{(t \nu \mathfrak{u} \eta)^{4} / 4}(\nu \mathfrak{u} \eta)^{4} d x\right] \\
& =\lambda \int_{A_{r_{1}, r_{4}}} \phi_{\nu \mathfrak{u} \eta}(\nu \mathfrak{u} \eta)^{2} d x-\liminf _{t \rightarrow \infty} \int_{A_{r_{1}, r_{4}}} \frac{F(t \nu \mathfrak{u} \eta)}{(t \nu \mathfrak{u} \eta)^{4} / 4}(\nu \mathfrak{u} \eta)^{4} d x \\
& \leq \lambda \int_{A_{r_{1}, r_{4}}} \phi_{\nu \mathfrak{u} \eta}(\nu \mathfrak{u} \eta)^{2} d x-\int_{A_{r_{1}, r_{4}}}\left[\liminf _{t \rightarrow \infty} \frac{F(t \nu \mathfrak{u} \eta)}{(t \nu \mathfrak{u} \eta)^{4} / 4}\right](\nu \mathfrak{u} \eta)^{4} d x \\
& =\lambda \int_{A_{r_{1}, r_{4}}} \phi_{\nu \mathfrak{u} \eta}(\nu \mathfrak{u} \eta)^{2} d x-\int_{A_{r_{1}, r_{4}}}(\nu \mathfrak{u} \eta)^{4} d x
\end{aligned}
$$

where the last inequality follows from Fatou's lemma. Therefore

$$
\begin{aligned}
\limsup _{t \rightarrow \infty} \frac{I_{\mathbb{R}^{3} \backslash B_{R}}\left(e_{t}\right)}{t^{4} / 4} \leq \int_{A_{r_{1}, r_{2}}}\left(\lambda \phi_{\nu \mathfrak{u}}-\nu^{2} \mathfrak{u}^{2}\right) \nu^{2} \mathfrak{u}^{2} d x+\int_{A_{r_{2}, r_{3}}}\left(\lambda \phi_{\mathfrak{u}}-\mathfrak{u}^{2}\right) \mathfrak{u}^{2} d x & \\
& +\int_{A_{r_{3}, r_{4}}}\left(\lambda \phi_{\mathfrak{u} \eta}-\mathfrak{u}^{2} \eta^{2}\right) \mathfrak{u}^{2} \eta^{2} d x .
\end{aligned}
$$

Now we have the estimate

$$
\begin{aligned}
\left|\int_{A_{r_{1}, r_{2}}}\left(\lambda \phi_{\nu \mathfrak{u}}-\nu^{2} \mathfrak{u}^{2}\right) \nu^{2} \mathfrak{u}^{2} d x\right| & \leq \int_{A_{r_{1}, r_{2}}}\left|\lambda \phi_{\nu \mathfrak{u}}-\nu^{2} \mathfrak{u}^{2}\right| \nu^{2} \mathfrak{u}^{2} d x \\
& \leq \int_{A_{r_{1}, r_{2}}}\left(\mathfrak{u}^{2}+\lambda \phi_{\mathfrak{u}}\right) \mathfrak{u}^{2} d x \\
& <\frac{\delta}{4}
\end{aligned}
$$

Similarly, we have

$$
\left|\int_{A_{r_{3}, r_{4}}}\left(\lambda \phi_{\mathfrak{u} \eta}-\mathfrak{u}^{2} \eta^{2}\right) \mathfrak{u}^{2} \eta^{2} d x\right|<\frac{\delta}{4}
$$

and hence

$$
\limsup _{t \rightarrow \infty} \frac{I_{\mathbb{R}^{3} \backslash B_{R}}\left(e_{t}\right)}{t^{4} / 4}<\frac{\delta}{4}-\delta+\frac{\delta}{4}=-\frac{\delta}{2},
$$

$\lim \sup _{t \rightarrow \infty} I_{\mathbb{R}^{3} \backslash B_{R}}\left(e_{t}\right)=-\infty$. Then to deduce (ii) it is sufficient to choose $T>0$ large enough, and set $e:=e_{T}$.

Remark 3.3.2. Observe that $\lambda$ did not enter explicit in the above proof; his smallness was used just to deduce the existence of $\mathfrak{u}$, see Lemma 3.2.1 and then to prove Lemma 3.3.1; however we already know that the smallness of $\lambda$ is a necessary condition in order to have a nontrivial solution.

Since the Mountain Pass geometry holds for the functional $I_{\mathbb{R}^{3} \backslash B_{R}}$, we can define the minimax level

$$
c_{\mathbb{R}^{3} \backslash B_{R}}:=\inf _{\gamma \in \Gamma} \max _{t \in[0,1]} I_{\mathbb{R}^{3} \backslash B_{R}}(\gamma(t)) \geq b>0,
$$

where

$$
\Gamma:=\left\{\gamma \in C\left([0,1], H_{0, r a d}^{1}\left(\mathbb{R}^{3} \backslash B_{R}\right)\right): \gamma(0)=0 \text { and } I_{\mathbb{R}^{3} \backslash B_{R}}(\gamma(1))<0\right\},
$$

which is not empty by Lemma 3.3.1. By Ekeland's Variational Principle, there exists a Cerami sequence at level $c_{\mathbb{R}^{3} \backslash B_{R}}$, i.e., $\left\{u_{n}\right\} \subset H_{0, \text { rad }}^{1}\left(\mathbb{R}^{3} \backslash B_{R}\right)$ such that

$$
I_{\mathbb{R}^{3} \backslash B_{R}}\left(u_{n}\right) \rightarrow c_{\mathbb{R}^{3} \backslash B_{R}} \quad \text { and } \quad\left\|I_{\mathbb{R}^{3} \backslash B_{R}}^{\prime}\left(u_{n}\right)\right\|_{\star}\left(1+\left\|u_{n}\right\|_{\mathbb{R}^{3} \backslash B_{R}}\right) \rightarrow 0, \quad \text { when } n \rightarrow \infty,
$$

where $\|\cdot\|_{\star}$ denotes the norm in the dual space of $H_{0, \text { rad }}^{1}\left(\mathbb{R}^{3} \backslash B_{R}\right)$. In the remaining part of this 
Section, $\left\{u_{n}\right\}$ will be the Cerami sequence whose existence is guaranteed above and $\left\{\widetilde{u}_{n}\right\}$ as usual its extension to $\mathbb{R}^{3}$.

\subsubsection{Compactness condition for $I_{\mathbb{R}^{3} \backslash B_{R}}$}

We first prove the a priori estimate on the Cerami sequence.

Lemma 3.3.3. The Cerami sequence $\left\{u_{n}\right\}$ is bounded.

Proof. Assume by contradiction that there exists a subsequence (denoted again by $\left\{u_{n}\right\}$ ) such that $\left\|u_{n}\right\|_{\mathbb{R}^{3} \backslash B_{R}} \rightarrow \infty$ when $n \rightarrow \infty$. We define

$$
v_{n}:=2 \sqrt{c_{\mathbb{R}^{3} \backslash B_{R}}} \frac{u_{n}}{\left\|u_{n}\right\|_{\mathbb{R}^{3} \backslash B_{R}}} .
$$

Clearly $\left\{v_{n}\right\} \subset H_{0, \text { rad }}^{1}\left(\mathbb{R}^{3} \backslash B_{R}\right)$ and $\left\|\widetilde{v}_{n}\right\|=\left\|v_{n}\right\|_{\mathbb{R}^{3} \backslash B_{R}}=2 \sqrt{c_{\mathbb{R}^{3} \backslash B_{R}}}$.

Claim: there are positive numbers $r$ and $d$ and a sequence $\left\{y_{n}\right\} \subset \mathbb{R}^{3}$ such that

$$
\liminf _{n \rightarrow \infty} \int_{B_{r}\left(y_{n}\right)} \widetilde{v}_{n}^{2} d x \geq d>0
$$

Indeed, assume that the claim does not hold. Then

$$
\lim _{n \rightarrow \infty} \sup _{y \in \mathbb{R}^{3}} \int_{B_{r}(y)} \widetilde{v}_{n}^{2} d x=0,
$$

and by Lion's lemma, $\widetilde{v}_{n} \rightarrow 0$ in $L^{q}\left(\mathbb{R}^{3}\right)$, for every $2<q<6$. From (3.1.3)

$$
\left|\int_{\mathbb{R}^{3}} F\left(\widetilde{v}_{n}\right) d x\right| \leq \frac{\varepsilon}{2} \int_{\mathbb{R}^{3}} \widetilde{v}_{n}^{2} d x+\frac{M_{\varepsilon}}{q} \int_{\mathbb{R}^{3}}\left|\widetilde{v}_{n}\right|^{q} d x,
$$

and thus we have

$$
\lim _{n \rightarrow \infty} \int_{\mathbb{R}^{3}} F\left(\widetilde{v}_{n}\right) d x=0
$$

Consequently

$$
I\left(\widetilde{v}_{n}\right)=\frac{1}{2}\left\|v_{n}\right\|_{\mathbb{R}^{3} \backslash B_{R}}^{2}+\frac{\lambda}{4} \int_{\mathbb{R}^{3}} \phi_{\widetilde{v}_{n}} \widetilde{v}_{n}^{2} d x-\int_{\mathbb{R}^{3}} F\left(\widetilde{v}_{n}\right) d x=2 c_{\mathbb{R}^{3} \backslash B_{R}}+\frac{\lambda}{4} \int_{\mathbb{R}^{3}} \phi_{\widetilde{v}_{n}} \widetilde{v}_{n}^{2} d x+o_{n}(1) .
$$

Since $\left\{u_{n}\right\}$ is a Cerami sequence,

$$
I_{\mathbb{R}^{3} \backslash B_{R}}\left(u_{n}\right)=c_{\mathbb{R}^{3} \backslash B_{R}}+o_{n}(1) \quad \text { and } \quad\left\|I_{\mathbb{R}^{3} \backslash B_{R}}^{\prime}\left(u_{n}\right)\right\|_{\star}\left\|u_{n}\right\|_{\mathbb{R}^{3} \backslash B_{R}}<1 / n
$$

and this last statement implies

$$
-\frac{1}{n}<I_{\mathbb{R}^{3} \backslash B_{R}}^{\prime}\left(u_{n}\right)\left[u_{n}\right]=\left\|u_{n}\right\|_{\mathbb{R}^{3} \backslash B_{R}}^{2}+\lambda \int_{\mathbb{R}^{3} \backslash B_{R}} \phi_{\widetilde{u}_{n}} u_{n}^{2} d x-\int_{\mathbb{R}^{3} \backslash B_{R}} f\left(u_{n}\right) u_{n} d x<\frac{1}{n} .
$$

Let us introduce now the function

$$
\xi(t):=\left(\frac{t^{2}}{2}-\frac{t^{4}}{4}\right)\left[\left|\nabla u_{n}\right|^{2}+u_{n}^{2}\right]+\frac{t^{4}}{4} f\left(u_{n}\right) u_{n}-F\left(t u_{n}\right), \quad t \geq 0 .
$$

For any $t>0$, in the set $\left\{x \in \mathbb{R}^{3}: u_{n}(x) \neq 0\right\}$ which has positive measure, we have

$$
\begin{aligned}
\xi^{\prime}(t) & =\left(t-t^{3}\right)\left[\left|\nabla u_{n}\right|^{2}+u_{n}^{2}\right]+t^{3} f\left(u_{n}\right) u_{n}-f\left(t u_{n}\right) u_{n} \\
& =t\left(1-t^{2}\right)\left[\left|\nabla u_{n}\right|^{2}+u_{n}^{2}\right]+t^{3} u_{n}^{4}\left[\frac{f\left(u_{n}\right)}{u_{n}^{3}}-\frac{f\left(t u_{n}\right)}{t^{3} u_{n}^{3}}\right] .
\end{aligned}
$$


By using (f5), the function $\xi$ has a maximum at $t=1$. Therefore

$$
\xi(t) \leq \frac{1}{4}\left[\left|\nabla u_{n}\right|^{2}+u_{n}^{2}\right]+\frac{1}{4} f\left(u_{n}\right) u_{n}-F\left(u_{n}\right), \quad t \geq 0 .
$$

Integrating on $\mathbb{R}^{3} \backslash B_{R}$ this last inequality we get

$$
\begin{aligned}
&\left(\frac{t^{2}}{2}-\frac{t^{4}}{4}\right)\left\|u_{n}\right\|_{\mathbb{R}^{3} \backslash B_{R}}^{2}+\int_{\mathbb{R}^{3} \backslash B_{R}}\left[\frac{t^{4}}{4} f\left(u_{n}\right) u_{n}-F\left(t u_{n}\right)\right] d x \leq \\
& \frac{1}{4}\left\|u_{n}\right\|_{\mathbb{R}^{3} \backslash B_{R}}^{2}+\int_{\mathbb{R}^{3} \backslash B_{R}}\left[\frac{1}{4} f\left(u_{n}\right) u_{n}-F\left(u_{n}\right)\right] d x .
\end{aligned}
$$

Now by making use of (3.3.4) and (3.3.5), we infer

$$
\begin{aligned}
I_{\mathbb{R}^{3} \backslash B_{R}}\left(t u_{n}\right) & =\frac{t^{2}}{2}\left\|u_{n}\right\|_{\mathbb{R}^{3} \backslash B_{R}}^{2}+\frac{t^{4}}{4} \lambda \int_{\mathbb{R}^{3} \backslash B_{R}} \phi_{\widetilde{u}_{n}} u_{n}^{2} d x-\int_{\mathbb{R}^{3} \backslash B_{R}} F\left(t u_{n}\right) d x \\
& <\frac{t^{2}}{2}\left\|u_{n}\right\|_{\mathbb{R}^{3} \backslash B_{R}}^{2}+\frac{t^{4}}{4}\left[\frac{1}{n}+\int_{\mathbb{R}^{3} \backslash B_{R}} f\left(u_{n}\right) u_{n} d x-\left\|u_{n}\right\|_{\mathbb{R}^{3} \backslash B_{R}}^{2}\right]-\int_{\mathbb{R}^{3} \backslash B_{R}} F\left(t u_{n}\right) d x \\
& =\left(\frac{t^{2}}{2}-\frac{t^{4}}{4}\right)\left\|u_{n}\right\|_{\mathbb{R}^{3} \backslash B_{R}}^{2}+\frac{t^{4}}{4 n}+\int_{\mathbb{R}^{3} \backslash B_{R}}\left[\frac{t^{4}}{4} f\left(u_{n}\right) u_{n}-F\left(t u_{n}\right)\right] d x \\
& \leq \frac{t^{4}}{4 n}+\frac{1}{4}\left\|u_{n}\right\|_{\mathbb{R}^{3} \backslash B_{R}}^{2}+\int_{\mathbb{R}^{3} \backslash B_{R}}\left[\frac{1}{4} f\left(u_{n}\right) u_{n}-F\left(u_{n}\right)\right] d x,
\end{aligned}
$$

namely

$$
I_{\mathbb{R}^{3} \backslash B_{R}}\left(t u_{n}\right)<\frac{t^{4}}{4 n}+\frac{1}{4}\left\|u_{n}\right\|_{\mathbb{R}^{3} \backslash B_{R}}^{2}+\int_{\mathbb{R}^{3} \backslash B_{R}}\left[\frac{1}{4} f\left(u_{n}\right) u_{n}-F\left(u_{n}\right)\right] d x, \quad \forall t>0 .
$$

By using (3.3.4) again, we get

$$
\begin{aligned}
I_{\mathbb{R}^{3} \backslash B_{R}}\left(u_{n}\right) & >\frac{1}{2}\left\|u_{n}\right\|_{\mathbb{R}^{3} \backslash B_{R}}^{2}-\int_{\mathbb{R}^{3} \backslash B_{R}} F\left(u_{n}\right) d x-\frac{1}{4 n}+\int_{\mathbb{R}^{3} \backslash B_{R}} \frac{1}{4} f\left(u_{n}\right) u_{n} d x-\frac{1}{4}\left\|u_{n}\right\|_{\mathbb{R}^{3} \backslash B_{R}}^{2} \\
& =\frac{1}{4}\left\|u_{n}\right\|_{\mathbb{R}^{3} \backslash B_{R}}^{2}+\int_{\mathbb{R}^{3} \backslash B_{R}}\left[\frac{1}{4} f\left(u_{n}\right) u_{n}-F\left(u_{n}\right)\right] d x-\frac{1}{4 n} .
\end{aligned}
$$

By (3.3.6) and (3.3.7) we deduce

$$
I_{\mathbb{R}^{3} \backslash B_{R}}\left(t u_{n}\right)<\frac{t^{4}}{4 n}+I_{\mathbb{R}^{3} \backslash B_{R}}\left(u_{n}\right)+\frac{1}{4 n}, \quad \forall t>0 .
$$

In particular by taking $t=t_{n}:=2 \sqrt{c_{\mathbb{R}^{3} \backslash B_{R}}} /\left\|u_{n}\right\|_{\mathbb{R}^{3} \backslash B_{R}}$ we obtain

$$
\begin{aligned}
I_{\mathbb{R}^{3} \backslash B_{R}}\left(v_{n}\right) & <\frac{2^{4}\left(\sqrt{c_{\mathbb{R}^{3} \backslash B_{R}}}\right)^{4}}{4 n\left\|u_{n}\right\|_{\mathbb{R}^{3} \backslash B_{R}}^{4}}+I_{\mathbb{R}^{3} \backslash B_{R}}\left(u_{n}\right)+\frac{1}{4 n} \\
& =\frac{4 c_{\mathbb{R}^{3} \backslash B_{R}}^{2}}{n\left\|u_{n}\right\|_{\mathbb{R}^{3} \backslash B_{R}}^{4}}+\frac{1}{4 n}+I_{\mathbb{R}^{3} \backslash B_{R}}\left(u_{n}\right) \\
& =c_{\mathbb{R}^{3} \backslash B_{R}}+o_{n}(1)
\end{aligned}
$$


Hence, combining (3.3.3) and (3.3.8) we arrive at

$$
\begin{aligned}
2 c_{\mathbb{R}^{3} \backslash B_{R}}+o_{n}(1) & <2 c_{\mathbb{R}^{3} \backslash B_{R}}+\frac{\lambda}{4} \int_{\mathbb{R}^{3}} \phi_{\widetilde{v}_{n}} \widetilde{v}_{n}^{2} d x+o_{n}(1) \\
& =I\left(\widetilde{v}_{n}\right) \\
& =I_{\mathbb{R}^{3} \backslash B_{R}}\left(v_{n}\right) \\
& <c_{\mathbb{R}^{3} \backslash B_{R}}+o_{n}(1),
\end{aligned}
$$

and this contradiction proves the claim (3.3.2).

Two cases now are possible.

Case 1: $\left\{y_{n}\right\}$ is bounded.

Then there is $\widehat{r}>0$ such that $\left\{y_{n}\right\} \subset B_{\widehat{r}}$. By (3.3.2) we have $\int_{B_{r}\left(y_{n}\right)} \widetilde{v}_{n}^{2} d x>\frac{d}{2}$. Thus, we can choose $\widetilde{r}>\widehat{r}+r$ such that $B_{r}\left(y_{n}\right) \subset B_{\widetilde{r}}$ and hence

$$
\int_{B_{\widetilde{r}}} \widetilde{v}_{n}^{2} d x>\frac{d}{2}
$$

Since $\left\|\widetilde{v}_{n}\right\|=2 \sqrt{c_{\mathbb{R}^{3} \backslash B_{R}}}$, there is a subsequence still denoted by $\left\{\widetilde{v}_{n}\right\}$ such that $\widetilde{v}_{n} \rightarrow v$ in $H^{1}\left(\mathbb{R}^{3}\right)$, from which $\widetilde{v}_{n} \rightarrow v$ in $L_{l o c}^{q}\left(\mathbb{R}^{3}\right)$, for $2 \leq q<6$, and $\widetilde{v}_{n}(x) \rightarrow v(x)$ a.e. $x \in \mathbb{R}^{3}$. In particular

$$
\int_{B_{\widetilde{r}}} \widetilde{v}_{n}^{2} d x \rightarrow \int_{B_{\widetilde{r}}} v^{2} d x, \text { and thus } \int_{B_{\widetilde{r}}} v^{2} d x \geq \frac{d}{2}>0
$$

implying that $v \neq 0$. But then there is $\Lambda \subset B_{\widetilde{r}} \cap\left(\mathbb{R}^{3} \backslash B_{R}\right)$ with meas $(\Lambda)>0$ and such that $v(x) \neq 0$, for every $x \in \Lambda$. Thence for $x \in \Lambda$ fixed, $v_{n}(x)=2 \sqrt{c_{\mathbb{R}^{3} \backslash B_{R}}} u_{n}(x) /\left\|u_{n}\right\|_{\Omega} \neq 0$ for $n$ large enough, so that we infer that, for $n$ large $u_{n}(x) \neq 0$. Since $\left\|u_{n}\right\|_{\mathbb{R}^{3} \backslash B_{R}} \rightarrow \infty$, it is also $\left|u_{n}(x)\right| \rightarrow \infty$ and by the arbitrariness of $x,\left|u_{n}(x)\right| \rightarrow \infty$ for every $x \in \Lambda$. Since

$$
\begin{aligned}
I_{\mathbb{R}^{3} \backslash B_{R}}\left(u_{n}\right)-\frac{1}{4} I_{\mathbb{R}^{3} \backslash B_{R}}^{\prime}\left(u_{n}\right)\left[u_{n}\right] & =\frac{1}{4}\left\|u_{n}\right\|_{\mathbb{R}^{3} \backslash B_{R}}^{2}-\int_{\mathbb{R}^{3} \backslash B_{R}} F\left(u_{n}\right) d x+\frac{1}{4} \int_{\mathbb{R}^{3} \backslash B_{R}} f\left(u_{n}\right) u_{n} d x \\
& \geq \int_{\mathbb{R}^{3} \backslash B_{R}}\left[\frac{1}{4} f\left(u_{n}\right) u_{n}-F\left(u_{n}\right)\right] d x \\
& \geq \int_{\Lambda}\left[\frac{1}{4} f\left(u_{n}\right) u_{n}-F\left(u_{n}\right)\right] d x,
\end{aligned}
$$

by (f6) and the Fatou's lemma

$$
\begin{aligned}
\liminf _{n \rightarrow \infty}\left[I_{\mathbb{R}^{3} \backslash B_{R}}\left(u_{n}\right)-\frac{1}{4} I_{\mathbb{R}^{3} \backslash B_{R}}^{\prime}\left(u_{n}\right)\left[u_{n}\right]\right] & \geq \liminf _{n \rightarrow \infty} \int_{\Lambda}\left[\frac{1}{4} f\left(u_{n}\right) u_{n}-F\left(u_{n}\right)\right] d x \\
& \geq \int_{\Lambda} \liminf _{n \rightarrow \infty}\left[\frac{1}{4} f\left(u_{n}\right) u_{n}-F\left(u_{n}\right)\right] d x \\
& =\infty,
\end{aligned}
$$

in contradiction with the fact that

$$
I_{\mathbb{R}^{3} \backslash B_{R}}\left(u_{n}\right)-\frac{1}{4} I_{\mathbb{R}^{3} \backslash B_{R}}^{\prime}\left(u_{n}\right)\left[u_{n}\right]=c_{\mathbb{R}^{3} \backslash B_{R}}+o_{n}(1) .
$$

Then Case 1 does not hold.

Case 2: $\left|y_{n}\right| \rightarrow \infty$ when $n \rightarrow \infty$.

We define $\widehat{v}_{n}:=\widetilde{v}_{n}\left(\cdot+y_{n}\right)$; it is clear that $\left\|\widehat{v}_{n}\right\|=\left\|\widetilde{v}_{n}\right\|=2 \sqrt{c_{\mathbb{R}^{3} \backslash B_{R}}}$. Thus, up to a subsequence, $\widehat{v}_{n} \rightarrow \widehat{v}$ in $H^{1}\left(\mathbb{R}^{3}\right), \widehat{v}_{n} \rightarrow \widehat{v}$ in $L_{l o c}^{q}\left(\mathbb{R}^{3}\right)$ for $2 \leq q<6$, and $\widehat{v}_{n}(x) \rightarrow \widehat{v}(x)$ a.e. $x \in \mathbb{R}^{3}$. From (3.3.2) 
it holds

$$
\liminf _{n \rightarrow \infty} \int_{B_{r}\left(y_{n}\right)} \widetilde{v}_{n}^{2} d x=\liminf _{n \rightarrow \infty} \int_{B_{r}} \widehat{v}_{n}^{2} d x \geq d
$$

and hence

$$
\int_{B_{r}} \widehat{v}^{2} d x \geq d>0
$$

which implies that $\widehat{v} \neq 0$. But then there is $\Lambda \subset B_{r}$ with meas $(\Lambda)>0$ such that $\widehat{v}(x) \neq 0$, for every $x \in \Lambda$. Thence for $x \in \Lambda$ fixed,

$$
\widehat{v}_{n}(x)=2 \sqrt{c_{\mathbb{R}^{3} \backslash B_{R}}} \widetilde{u}_{n}\left(x+y_{n}\right) /\left\|u_{n}\right\|_{\mathbb{R}^{3} \backslash B_{R}} \neq 0 \quad \text { for large } n,
$$

so that we get $\widetilde{u}_{n}\left(x+y_{n}\right) \neq 0$. Since $\left\|u_{n}\right\|_{\mathbb{R}^{3} \backslash B_{R}} \rightarrow \infty$, we have $\left|\widetilde{u}_{n}\left(x+y_{n}\right)\right| \rightarrow \infty$. By the arbitrariness of $x,\left|\widetilde{u}_{n}\left(x+y_{n}\right)\right| \rightarrow \infty$ for every $x \in \Lambda$. Furthermore, since $\left|y_{n}\right| \rightarrow \infty$, we can assume that $B_{r}\left(y_{n}\right) \subset\left(\mathbb{R}^{3} \backslash B_{R}\right)$, for every $n$. Therefore

$$
\begin{aligned}
I_{\mathbb{R}^{3} \backslash B_{R}}\left(u_{n}\right)-\frac{1}{4} I_{\mathbb{R}^{3} \backslash B_{R}}^{\prime}\left(u_{n}\right)\left[u_{n}\right] & =\frac{1}{4}\left\|u_{n}\right\|_{\mathbb{R}^{3} \backslash B_{R}}^{2}-\int_{\mathbb{R}^{3} \backslash B_{R}} F\left(u_{n}\right) d x+\frac{1}{4} \int_{\mathbb{R}^{3} \backslash B_{R}} f\left(u_{n}\right) u_{n} d x \\
& \geq \int_{\mathbb{R}^{3} \backslash B_{R}}\left[\frac{1}{4} f\left(u_{n}\right) u_{n}-F\left(u_{n}\right)\right] d x \\
& \geq \int_{B_{r}\left(y_{n}\right)}\left[\frac{1}{4} f\left(u_{n}\right) u_{n}-F\left(u_{n}\right)\right] d x \\
& =\int_{B_{r}}\left[\frac{1}{4} f\left(u_{n}\left(x+y_{n}\right)\right) u_{n}\left(x+y_{n}\right)-F\left(u_{n}\left(x+y_{n}\right)\right)\right] d x \\
& \geq \int_{\Lambda}\left[\frac{1}{4} f\left(u_{n}\left(x+y_{n}\right)\right) u_{n}\left(x+y_{n}\right)-F\left(u_{n}\left(x+y_{n}\right)\right)\right] d x,
\end{aligned}
$$

and using (f6) and the Fatou's lemma

$$
\begin{aligned}
\liminf _{n \rightarrow \infty} & {\left[I_{\mathbb{R}^{3} \backslash B_{R}}\left(u_{n}\right)-\frac{1}{4} I_{\mathbb{R}^{3} \backslash B_{R}}^{\prime}\left(u_{n}\right)\left[u_{n}\right]\right] } \\
& \geq \int_{\Lambda} \liminf _{n \rightarrow \infty}\left[\frac{1}{4} f\left(u_{n}\left(x+y_{n}\right)\right) u_{n}\left(x+y_{n}\right)-F\left(u_{n}\left(x+y_{n}\right)\right)\right] d x=\infty,
\end{aligned}
$$

in contradiction with $I_{\mathbb{R}^{3} \backslash B_{R}}\left(u_{n}\right)-\frac{1}{4} I_{\mathbb{R}^{3} \backslash B_{R}}^{\prime}\left(u_{n}\right)\left[u_{n}\right]=c_{\mathbb{R}^{3} \backslash B_{R}}+o_{n}(1)$. Then also Case 2 cannot hold.

Then the proof is thereby completed.

Now we are ready to prove the result about the existence of positive radial solutions for (3.3.1).

\subsubsection{Proof of Theorem 3.1.1}

Actually we will prove that the Cerami sequence $\left\{u_{n}\right\}$ given above admits a convergent subsequence.

By Lemma 3.3.3, we can assume that the Cerami sequence $u_{n} \rightarrow u$ in $H_{0, \text { rad }}^{1}\left(\mathbb{R}^{3} \backslash B_{R}\right)$ and then $u_{n} \rightarrow u$ in $L^{p}\left(\mathbb{R}^{3} \backslash B_{R}\right), 2<p<6$. Let us see that $I_{\mathbb{R}^{3} \backslash B_{R}}^{\prime}(u)=0$.

Note that $u_{n} \rightarrow u$ in $H_{0, \text { rad }}^{1}\left(\mathbb{R}^{3} \backslash B_{R}\right)$ implies $\widetilde{u}_{n} \rightarrow \widetilde{u}$ in $H_{\text {rad }}^{1}\left(\mathbb{R}^{3}\right)$. For every $w \in H_{0, \text { rad }}^{1}\left(\mathbb{R}^{3} \backslash B_{R}\right)$ by using the compact embedding and the arguments in Ruiz (2005, Lemma 2.1), we have

$$
\int_{\mathbb{R}^{3} \backslash B_{R}} f\left(u_{n}\right) w d x \rightarrow \int_{\mathbb{R}^{3} \backslash B_{R}} f(u) w d x \quad \text { and } \quad \int_{\mathbb{R}^{3} \backslash B_{R}} \phi_{\widetilde{u}_{n}} u_{n} w d x \rightarrow \int_{\mathbb{R}^{3} \backslash B_{R}} \phi_{\widetilde{u}} u w d x
$$

from which $I_{\mathbb{R}^{3} \backslash B_{R}}^{\prime}\left(u_{n}\right)[w] \rightarrow I_{\mathbb{R}^{3} \backslash B_{R}}^{\prime}(u)[w]$. But $I_{\mathbb{R}^{3} \backslash B_{R}}^{\prime}\left(u_{n}\right) \rightarrow 0$, so that $I_{\mathbb{R}^{3} \backslash B_{R}}^{\prime}(u)=0$. To show 
the strong convergence, we note that

$$
\int_{\mathbb{R}^{3} \backslash B_{R}} f\left(u_{n}\right) u_{n} d x \rightarrow \int_{\mathbb{R}^{3} \backslash B_{R}} f(u) u d x \quad \text { and } \quad \int_{\mathbb{R}^{3} \backslash B_{R}} \phi_{\widetilde{u}_{n}} u_{n}^{2} d x \rightarrow \int_{\mathbb{R}^{3} \backslash B_{R}} \phi_{\widetilde{u}} u^{2} d x
$$

that together with previous claim and

$$
\left\|u_{n}\right\|_{\mathbb{R}^{3} \backslash B_{R}}^{2}+\int_{\mathbb{R}^{3} \backslash B_{R}} \phi_{\widetilde{u}_{n}} u_{n}^{2} d x=\int_{\mathbb{R}^{3} \backslash B_{R}} f\left(u_{n}\right) u_{n} d x+o_{n}(1)
$$

give us $\left\|u_{n}\right\|_{\mathbb{R}^{3} \backslash B_{R}} \rightarrow\|u\|_{\mathbb{R}^{3} \backslash B_{R}}$. We know that this solution is positive.

\subsection{A positive radial solution inside the ball}

Now we are interested in positive radial solutions inside the ball $B_{R}$, with $R>0$ fixed, for the problem

$$
\left\{\begin{array}{lc}
-\Delta u+u+\lambda \phi u=f(u) & \text { in } B_{R}, \\
-\Delta \phi=\widetilde{u}^{2} & \text { in } \mathbb{R}^{3}, \\
u=0 & \text { on } \partial B_{R}
\end{array}\right.
$$

We can write a $C^{1}$ functional on $H_{0, \text { rad }}^{1}\left(B_{R}\right)$,

$$
I_{B_{R}}(u)=\frac{1}{2} \int_{B_{R}}|\nabla u|^{2} d x+\frac{1}{2} \int_{B_{R}} u^{2} d x+\frac{\lambda}{4} \int_{B_{R}} \phi_{\widetilde{u}} u^{2} d x-\int_{B_{R}} F(u) d x .
$$

whose critical points are radial solutions of (3.4.1). $I_{B_{R}}$. Now it is simpler than in the case of unbounded domain, but we give the details for the reader convenience.

All that we will say in the following will be true for any value of $R, \lambda>0$. In this section we use the notation

$$
\|u\|_{H_{0}^{1}\left(B_{R}\right)}^{2}:=\int_{B_{R}}|\nabla u|^{2} d x
$$

for the (square) equivalent norm in $H_{0, r a d}^{1}\left(B_{R}\right)$.

\subsubsection{Mountain Pass geometry of $I_{B_{R}}$}

Again the most involved part is to show the existence of a point with large norm where the functional is negative.

Lemma 3.4.1. Assume that (f1),(f3) and (f4) are satisfied. Then there exist positive numbers $b, \rho$ and $e \in H_{0, \text { rad }}^{1}\left(B_{R}\right)$ such that

(i) $I_{B_{R}}(u) \geq b$, for every $u \in H_{0, r a d}^{1}\left(B_{R}\right)$ such that $\|u\|_{H_{0}^{1}\left(B_{R}\right)}=\rho$;

(ii) $\|e\|_{H_{0}^{1}\left(B_{R}\right)}>\rho$ and $I_{B_{R}}(e)<0$.

Proof. Using (3.1.3) for $0<\varepsilon<1$ fixed, we have

$$
\begin{aligned}
I_{B_{R}}(u) & \geq \frac{1}{2}\|u\|_{H_{0}^{1}\left(B_{R}\right)}^{2}+\frac{1}{2} \int_{B_{R}} u^{2} d x-\int_{B_{R}} F(u) d x \\
& \geq \frac{1}{2}\|u\|_{H_{0}^{1}\left(B_{R}\right)}^{2}+\frac{1-\varepsilon}{2} \int_{B_{R}} u^{2} d x-\frac{M_{\varepsilon}}{q} \int_{B_{R}}|u|^{q} d x \\
& \geq \frac{1}{2}\|u\|_{H_{0}^{1}\left(B_{R}\right)}^{2}-M_{\varepsilon}\|u\|_{H_{0}^{1}\left(B_{R}\right)}^{q}>0,
\end{aligned}
$$

for $u \in H_{0, \text { rad }}^{1}\left(B_{R}\right)$ with norm sufficiently small. This gives (i). 
Now let $\eta$ be a $C_{c}^{\infty}\left(B_{R}\right)$ and positive function and define $\eta_{t}(x):=t^{2} \eta(t x)$, with $t>1$. Note that $\lim _{t \rightarrow+\infty}\left\|\eta_{t}\right\|_{H_{0}^{1}\left(B_{R}\right)}=+\infty$. In this paragraph, we will identify $\eta(t \cdot)$ and $\eta_{t}$, for $t>1$, with their trivial extensions to whole $B_{R}$. We will continue to denote with $\widetilde{\eta}, \widetilde{\eta}_{t}$ the extensions of $\eta, \eta_{t}$, respectively, to the whole $\mathbb{R}^{3}$. Then

$$
\begin{aligned}
I_{B_{R}}\left(\eta_{t}\right) & =\frac{1}{2} \int_{B_{R}}\left|\nabla \eta_{t}\right|^{2} d x+\frac{1}{2} \int_{B_{R}} \eta_{t}^{2} d x+\frac{\lambda}{4} \int_{B_{R}} \phi_{\widetilde{\eta}_{t}} \eta_{t}^{2} d x-\int_{B_{R}} F\left(\eta_{t}\right) d x \\
& =\frac{t^{3}}{2} \int_{B_{R}}|\nabla \eta|^{2} d x+\frac{t}{2} \int_{B_{R}} \eta^{2} d x+\frac{\lambda t^{3}}{4} \int_{B_{R}} \phi_{\widetilde{\eta}} \eta^{2} d x-\int_{B_{R}} \frac{F\left(t^{2} \eta\right)}{t^{3}} d x \\
& =\frac{t^{3}}{2}\left(\|\eta\|_{H_{0}^{1}\left(B_{R}\right)}^{2}+\frac{\lambda}{2} \int_{B_{R}} \phi_{\widetilde{\eta}} \eta^{2} d x\right)+\frac{t}{2} \int_{B_{R}} \eta^{2} d x-\frac{t^{5}}{4} \int_{\text {supp }(\eta)} \frac{F\left(t^{2} \eta\right)}{\left(t^{2} \eta\right)^{4} / 4} \eta^{4} d x
\end{aligned}
$$

Since by the Fatou's Lemma,

$$
\int_{\operatorname{supp}(\eta)} \eta^{4} d x \leq \liminf _{t \rightarrow \infty} \int_{\operatorname{supp}(\eta)} \frac{F\left(t^{2} \eta\right)}{\left(t^{2} \eta\right)^{4} / 4} \eta^{4} d x
$$

we get for $t>0$ large enough

$$
\frac{t^{2}}{4} \int_{\operatorname{supp}(\eta)} \frac{F\left(t^{2} \eta\right)}{\left(t^{2} \eta\right)^{4} / 4} \eta^{4} d x \geq \frac{t^{2}}{8} \liminf _{t \rightarrow \infty} \int_{\operatorname{supp}(\eta)} \frac{F\left(t^{2} \eta\right)}{\left(t^{2} \eta\right)^{4} / 4} \eta^{4} d x \geq \frac{t^{2}}{8} \int_{\operatorname{supp}(\eta)} \eta^{4} d x
$$

from which

$$
\limsup _{t \rightarrow \infty} \frac{I_{B_{R}}\left(\eta_{t}\right)}{t^{3}}=\|\eta\|_{H_{0}^{1}\left(B_{R}\right)}^{2}+\frac{\lambda}{2} \int_{B_{R}} \phi_{\widetilde{\eta}} \eta^{2} d x-\liminf _{t \rightarrow \infty}\left(\frac{t^{2}}{4} \int_{\text {supp }(\eta)} \frac{F\left(t^{2} \eta\right)}{\left(t^{2} \eta\right)^{4} / 4} \eta^{4} d x\right)=-\infty
$$

and (ii) is proved.

By the above result, we can define

$$
\begin{gathered}
c_{B_{R}}:=\inf _{\gamma \in \Gamma} \max _{t \in[0,1]} I_{B_{R}}(\gamma(t))>0, \\
\text { where } \Gamma=\left\{\gamma \in C\left([0,1], H_{0, \text { rad }}^{1}\left(B_{R}\right)\right): \gamma(0)=0 \text { and } I_{B_{R}}(\gamma(t))<0\right\} \neq \emptyset
\end{gathered}
$$

and there exists a Palais-Smale sequence at level $c_{B_{R}}$.

\subsubsection{Compactness condition for $I_{B_{R}}$}

Lemma 3.4.2. The functional $I_{B_{R}}$ satisfies the $(P S)$ condition.

The result is essentially known, but we give the proof for completeness.

Proof. Let $\left\{u_{n}\right\} \subset H_{0}^{1}\left(B_{R}\right)$ be a $(P S)$ sequence for $I_{B_{R}}$, i.e., $I_{B_{R}}^{\prime}\left(u_{n}\right) \rightarrow 0$ and $\left|I_{B_{R}}\left(u_{n}\right)\right| \leq M$, for some $M>0$ constant. Hence by (f6),

$$
\begin{aligned}
l_{n} & :=4 I_{B_{R}}\left(u_{n}\right)-I_{B_{R}}^{\prime}\left(u_{n}\right)\left[u_{n}\right] \\
& =\left\|u_{n}\right\|_{H_{0}^{1}\left(B_{R}\right)}^{2}+\int_{B_{R}} u_{n}^{2} d x+\int_{B_{R}}\left[f\left(u_{n}\right) u_{n}-4 F\left(u_{n}\right)\right] d x \\
& \geq\left\|u_{n}\right\|_{H_{0}^{1}\left(B_{R}\right)}^{2}
\end{aligned}
$$

On the other hand, $l_{n} \leq 4 M+L\left\|u_{n}\right\|_{H_{0}^{1}\left(B_{R}\right)}$, for $L>0$ constant. Then up to subsequence we can assume that $u_{n} \rightarrow u$, for some $u \in H_{0, \text { rad }}^{1}\left(B_{R}\right)$. 
For the strong convergence of $\left\{u_{n}\right\}$ in $H_{0}^{1}\left(B_{R}\right)$ we can argue as in Pisani and Siciliano (2008, Theorem 2): we know that

$$
\Delta u_{n}=u_{n}+\lambda\left(\left.\phi_{\widetilde{u}_{n}}\right|_{B_{R}}\right) u_{n}-f\left(u_{n}\right)-I_{B_{R}}^{\prime}\left(u_{n}\right)
$$

so it is sufficient to show that every sequence in the right hand side is bounded in $H^{-1}\left(B_{R}\right)$. The sequences $\left\{f\left(u_{n}\right)\right\}$ and $\left\{I_{B_{R}}^{\prime}\left(u_{n}\right)\right\}$ are convergent; the sequence $\left\{u_{n}\right\}$ is bounded. Let us show that also $\left\{\left(\left.\phi_{\widetilde{u}_{n}}\right|_{B_{R}}\right) u_{n}\right\}$ is bounded. In the following we denote by $|\cdot|_{p, B_{R}}$ the $L^{p}$-norm in the domain $B_{R}$. Indeed using (a2) we have

$$
\int_{B_{R}}\left|\nabla\left(\left.\phi_{\widetilde{u}_{n}}\right|_{B_{R}}\right)\right|^{2} d x \leq \int_{\mathbb{R}^{3}}\left|\nabla \phi_{\widetilde{u}_{n}}\right|^{2} d x=\int_{\mathbb{R}^{3}} \phi_{\widetilde{u}_{n}} \widetilde{u}_{n}^{2} d x \leq C\left\|\widetilde{u}_{n}\right\|^{4}=C\left\|u_{n}\right\|_{H_{0}^{1}\left(B_{R}\right)}^{4} \leq C .
$$

Also this implies, by the embedding $D^{1,2}\left(\mathbb{R}^{3}\right) \hookrightarrow L^{6}\left(\mathbb{R}^{3}\right)$, that $\left.\left|\phi_{\widetilde{u}_{n}}\right|_{B_{R}}\right|_{6, B_{R}} \leq C$. But since $L^{6}\left(B_{R}\right) \hookrightarrow L^{2}\left(B_{R}\right)$

$$
\int_{B_{R}}\left(\left.\phi_{\widetilde{u}_{n}}\right|_{B_{R}}\right)^{2} d x \leq\left. C\left|\phi_{\widetilde{u}_{n}}\right|_{B_{R}}\right|_{6, B_{R}} ^{6} \leq C
$$

From (3.4.2) and (3.4.3)

$$
\left\|\left.\phi_{\widetilde{u}_{n}}\right|_{B_{R}}\right\|_{H^{1}\left(B_{R}\right)}^{2} \leq C
$$

so that by the Sobolev embeddings

$$
\left|\left(\left.\phi_{\widetilde{u}_{n}}\right|_{B_{R}}\right) u_{n}\right|_{3 / 2, B_{R}} \leq\left.\left|\phi_{\widetilde{u}_{n}}\right|_{B_{R}}\right|_{3, B_{R}}\left|u_{n}\right|_{3, B_{R}} \leq C\left\|\left.\left.\phi_{\widetilde{u}_{n}}\right|_{B_{R}}\right|_{H^{1}\left(B_{R}\right)} ^{2}\right\| u_{n} \|_{H_{0}^{1}\left(B_{R}\right)} \leq C .
$$

Thus $\left\{\left(\left.\phi_{\widetilde{u}_{n}}\right|_{B_{R}}\right) u_{n}\right\}$ is bounded in $L^{3 / 2}\left(B_{R}\right)$ and therefore in $H^{-1}\left(B_{R}\right)$.

\subsubsection{Proof of Theorem 3.1.2}

By the Mountain Pass Theorem we have a solution of (3.4.1) and we know that this solution is positive. 


\section{Bibliography}

Alves et al.(2004) C. Alves, P. Carrião and E. Medeiros. Multiplicity of solutions for a class of quasilinear problem in exterior domains with Neumann conditions. Abs. Appl. Anal., 3:251-268. Cited in page. 3,39

Alves et al.(2017) C. Alves, M.A. Souto and S.H.M. Soares. A sign changing solution for the Schrödinger-Poisson equation in $\mathbb{R}^{3}$. Rocky Mountain J. of Math, 47(1):1-25. Cited in page. 50, 55

Alves and Figueiredo(2014a) C.O. Alves and G.M. Figueiredo. Multiple solutions for a semilinear elliptic equation with critical growth and magnetic field. Milan J. Math., 82(2):389-405. Cited in page. 29

Alves and Figueiredo(2014b) C.O. Alves and G.M. Figueiredo. Multiplicity of solutions for a NLS equations with magnetic fields in $\mathbb{R}^{N}$. Monatsh. Math., 175(1):1-23. Cited in page. 29

Alves and Figueiredo(2013) C.O. Alves and G.M. Figueiredo. Multiplicity of nontrivial solutions to a biharmonic equation via Lusternik-Schnirelman theory. Math. Methods Appl. Sci., 36(6): 683-694. Cited in page. 29

Alves and Figueiredo(2015) C.O. Alves and G.M. Figueiredo. Multiple solutions for a quasilinear Schrödinger equation on $\mathbb{R}^{N}$. Acta Appl. Math., 136:91-117. Cited in page. 29

Alves and Figueiredo(2006) C.O. Alves and G.M. Figueiredo. Existence and multiplicity of positive solutions to a p-Laplacian equation in $\mathbb{R}^{N}$. Differential and Integral Equations, 19: 143-162. Cited in page. 29, 39

Alves and Soares(2010) C.O. Alves and S.H.M. Soares. Multiplicity of positive solutions for a class of nonlinear Schrödinger equations. Adv. Differential Equations, 15:1083-1102. Cited in page. 29

Alves et al.(2009) C.O. Alves, G.M. Figueiredo and U. B. Severo. Multiplicity of positive solutions for a class of quasilinear problems. Adv. Differential Equations, 14(9-10):911-942. Cited in page. 29

Alves et al.(2012) C.O. Alves, G.M. Figueiredo and U. B. Severo. A result of multiplicity of solutions for a class of quasilinear equations. Proc. Edinb. Math. Soc., 55(2):291-309. Cited in page. 29

Ambrosetti and Malchiodi(2007) A. Ambrosetti and A. Malchiodi. Nonlinear analysis and semilinear elliptic problems. Cambridge Studies in Advanced Mathematics, 104. Cambridge University Press, Cambridge. Cited in page. 2, 7, 13, 14, 19, 24

Ambrosetti and Prodi(1995) A. Ambrosetti and G. Prodi. A primer of nonlinear analysis. Corrected reprint of the 1993 original. Cambridge Studies in Advanced Mathematics, 34. Cambridge University Press, Cambridge. Cited in page. 5

Ambrosetti and Rabinowitz(1973) A. Ambrosetti and P.H. Rabinowitz. Dual variational methods in critical point theory and applications. J. Functional Analysis, 14:349-381. Cited in page. 55 
Azzollini et al.(2010) A. Azzollini, P. d' Avenia and A. Pomponio. On the Schrödinger-Maxwell equations under the effect of a general nonlinear term. Ann. I. H. Poincaré, AN 27:779-791. Cited in page. 54

Badiale and Serra(2011) M. Badiale and E. Serra. Semilinear elliptic equations for beginners. Existence results via the variational approach. Universitext. Springer, London. Cited in page. 2, 4, 5

Benci and Cerami(1991) V. Benci and G. Cerami. The effect of the domain topology on the number of positive solutions of nonlinear elliptic problems. Arch. Rat. Mech. Anal., 114(1):79-93. Cited in page. 29

Benci and Fortunato(1998) V. Benci and D. Fortunato. An eigenvalue problem for the Schrödinger-Maxwell equations. Topol. Methods Nonlinear Anal., 11(2):283 - 293. Cited in page. 8, $10,27,49$

Benci and Fortunato(2014) V. Benci and D. Fortunato. Solitons in Schrödinger-Maxwell equations. J. Fixed Point Theory Appl., 15(1):101-132. Cited in page. 27

Benci et al.(1991) V. Benci, G. Cerami and D. Passaseo. On the number of the positive solutions of some nonlinear elliptic problems. Nonlinear Anal., Sc. Norm. Super. di Pisa Quaderni, Scuola Norm. Sup., Pisa, páginas 93-107. Cited in page. 29, 47

Benguria et al.(1981) R. Benguria, H. Brezis and E.-H. Lieb. The Thomas-Fermi-von Weizsäcker theory of atoms and molecules. Comm. Math. Phys., 79:167-180. Cited in page. 49

Caffarelli and Silvestre(2007) L. Caffarelli and L.E. Silvestre. An extension problem related to the fractional Laplacian. Comm. in Partial Differential Equations, 32(7-9):1245-1260. Cited in page. 28

Caffarelli and Valdinoci(2011) L. Caffarelli and E. Valdinoci. Uniform estimates and limiting arguments for nonlocal minimal surfaces. Calc. Var. Partial Diff. Equations, 41(1-2):203-240. Cited in page. 28

Caffarelli et al.(2010) L. Caffarelli, J.M. Roquejoffre and O. Savin. Nonlocal minimal surfaces. Comm. Pure Apple. Math., 63(9):1111-1144. Cited in page. 28

Catto and Lions(1992) I. Catto and P.-L. Lions. Binding of atoms and stability of molecules in Hartree and Thomas-Fermi type theories. part 1: A necessary and sufficient condition for the stability of general molecular system. Comm. Partial Differential Equations, 17:1051-1110. Cited in page. 49

Chang and del Mar González(2011) S.-Y. A. Chang and M. del Mar González. Fractional Laplacian in conformal geometry. Adv. Math., 226(2):1410-1432. Cited in page. 28

Cingolani and Lazzo(1997) S. Cingolani and M. Lazzo. Multiple semiclassical standing waves for a class of nonlinear Schrödinger equations. Top. Methods Nonlinear Anal., 10(1):1-13. Cited in page. 29

Cingolani and Lazzo(2000) S. Cingolani and M. Lazzo. Multiple positive solutions to nonlinear Schrödinger equations with competing potential functions. J. Diff. Equations, 160(1):118-138. Cited in page. 29

Cont and Tankov(2004) R. Cont and P. Tankov. Financial modelling with jump processes. Chapman\&Hall/CRC Financial Mathematics Series, Boca Raton, FL. Cited in page. 28

d'Avenia et al.(2015) P. d'Avenia, G. Siciliano and M. Squassina. On fractional Choquard equations. Math. Models Methods Appl. Sci., 25(8):1447-1476. Cited in page. 36 
Fang and Zhang(2011) Y. Fang and J. Zhang. Multiplicity of solutions for the nonlinear Schrödinger-Maxwell system. Communications on Pure and Applied Analysis, 10(4):1267-1279. Cited in page. 27, 32

Figueiredo and Siciliano(2016) G.M. Figueiredo and G. Siciliano. A multiplicity result via Ljusternick-Schnirelmann category and Morse theory for a fractional Schrödinger equation in $\mathbb{R}^{N}$. NoDEA Nonlinear Differ. Equ. Appl., 23(2):Art.12, 22pp. Cited in page. 29, 40, 46

Figueiredo et al.(2015) G.M. Figueiredo, Marcos T. Pimenta and G. Siciliano. Multiplicity results for the fractional Laplacian in expanded domains. arXiv1511.09406. Cited in page. 29

Ianni(2013) I. Ianni. Sign-changing radial solutions for the Schrödinger-Poisson-Slater problem. Topol. Methods Nonlinear Anal., 41(2):365-385. Cited in page. 50

Kavian(1993) O. Kavian. Introduction à la Théorie des Points Critiques. Springer-Verlag. Cited in page. 33

Kesavan(1989) S. Kesavan. Topics in functional analysis and applications. John Wiley \& Sons, Inc., New York. Cited in page. 11

Laskin(2000a) N. Laskin. Fractals and quantum mechanics. Chaos, 10(4):780-790. Cited in page. 28

Laskin(2000b) N. Laskin. Fractional quantum mechanics and Lévy path integrals. Phys. Lett. A, 268(4-6):298-305. Cited in page. 28

Laskin(2002) N. Laskin. Fractional Schrödinger equation. Phys. Rev. E, 66(5):056108, 7pp. Cited in page. 28

Liu(2010) S. Liu. On superlinear problems without the Ambrosetti and Rabinowitz condition. Nonlinear Analysis, 73:788-795. Cited in page. 52

Maia et al.(2015) L. Maia, O. Miyagaki and S. Soares. A sign-changing solution for an asymptotically linear Schrödinger equation. Proc. Edinb. Math. Soc., 2, 58(3):697-716. Cited in page. 51

Metzler and Klafter(2000) R. Metzler and J. Klafter. The random walk's guide to anomalous diffusion: a fractional dynamics approach. Phys. Rep., 339:1-77. Cited in page. 28

Metzler and Klafter(2004) R. Metzler and J. Klafter. The restaurant at the end of the random walk: recent developments in the description of anomalous transport by fractional dynamics. $J$. Phys. A, 37(31):R161-R208. Cited in page. 28

Milakis and Silvestre(2008) E. Milakis and L. Silvestre. Regularity for the nonlinear Signorini problem. Adv. Math., 217(3):1301-1312. Cited in page. 28

Munkres(2000) J. Munkres. Topology. Prentice-Hall, Inc., Englewood Cliffs, N.J., second edição. Cited in page. 20, 21

Nezza et al.(2012) E. Di Nezza, G. Palatucci and E. Valdinoci. Hitchhiker's guide to the fractional Sobolev spaces. Bull. Sci. Math., 136(5):521-573. Cited in page. 30

Pisani and Siciliano(2008) L. Pisani and G. Siciliano. Note on a Schrödinger-Poisson system in a bounded domain. Appl. Math. Lett., 21(5):521-528. Cited in page. 52, 64

Protter and Weinberger(1999) M. Protter and H. Weinberger. Maximum principles in differential equations. Springer-Verlag. Cited in page. 51 
Rabinowitz(1986) P. Rabinowitz. Minimax methods in critical point theory with applications to differential equations. CBMS Regional Conference Series in Mathematics, 65. Published for the Conference Board of the Mathematical Sciences, Washington, DC. American Mathematical Society, Providence, RI. Cited in page. 10

Royden(1988) H. Royden. Real Analysis. Macmillan Publishing Company, New York, third edição. Cited in page. 2

Ruiz(2005) D. Ruiz. Semiclassical states for coupled Schrödinger-Maxwell equations: concentration around a sphere. Math. Models Methods Appl. Sci., 15(1):141-164. Cited in page. 27, 61

Ruiz(2006) D. Ruiz. The Schrödinger-Poisson equation under the effect of a nonlinear local term. J. Funct. Anal., 237(2):655-674. Cited in page. 50, 53

Ruiz and Siciliano(2008) D. Ruiz and G. Siciliano. A note on the Schrödinger-Poisson-slater equation on bounded domains. Advanced Nonlinear Studies, 8:170-190. Cited in page. 50

Siciliano(2010) G. Siciliano. Multiple positive solutions for a Schrödinger-Poisson-Slater system. J. Math. Anal. Appl., 365:288-299. Cited in page. 27, 28

Silvestre(2007) L. Silvestre. Regularity of the obstacle problem for a fractional power of the Laplace operator. Comm. Pure Appl. Math., 60(1):67-112. Cited in page. 28

Stuart and Zhou(1999) C. Stuart and H.S. Zhou. Applying the mountain pass theorem to an asymptotically linear elliptic equation on $\mathbb{R}^{N}$. Comm. PDEs, 24(9-10):1731-1758. Cited in page. 56

Wei(2015) Z. Wei. Existence of infinitely many solutions for the fractional Schrödinger-Maxwell equations. arXiv1508.03088v1. Cited in page. 28

Willem(1996) M. Willem. Minimax theorems, volume 24. Birkhäuser. Cited in page. 10

Zhang(2015) J. Zhang. Existence and multiplicity results for the fractional Schrödinger-Poisson systems. arXiv150\%.01205v1. Cited in page. 28 